\title{
Total Synthesis of Kinamycins C, F, and J.
}

\author{
K. C. Nicolaou,
}

Contribution from the Department of Chemistry and The Skaggs Institute for Chemical Biology, The Scripps Research Institute, 10550 North Torrey Pines Road, La Jolla, California 92037, and Department of Chemistry and Biochemistry, University of California, San Diego, 9500 Gilman Drive, La Jolla, California 92093.

RECEIVED DATE (automatically inserted by publisher); kcn@scripps.edu

\section{Supporting Information Available}

\author{
I) Experimental Section $\quad \mathrm{S}-2$ \\ II) $\quad{ }^{1} \mathrm{H}$ and ${ }^{13} \mathrm{C}$ NMR of compounds 2-17, 1c, 1e, and 1f $\quad \mathrm{S}-20$
}

\section{Experimental Data}

\section{Experimental Data for Compounds}

General Procedures. All reactions were carried out under an argon atmosphere with dry solvents under anhydrous conditions, unless otherwise noted. Dry tetrahydrofuran (THF), toluene, diethyl ether $\left(\mathrm{Et}_{2} \mathrm{O}\right)$, and methylene chloride $\left(\mathrm{CH}_{2} \mathrm{Cl}_{2}\right)$, were obtained by passing commercially available pre-dried, oxygen-free formulations through activated alumina columns. Acetonitrile $\left(\mathrm{CH}_{3} \mathrm{CN}\right)$, dimethylsulfoxide (DMSO), and iso-propanol (i-PrOH) were purchased in anhydrous form and used without further purification. Yields refer to chromatographically and spectroscopically ( ${ }^{1} \mathrm{H}$ NMR) homogeneous materials, unless otherwise stated. Reagents were purchased at the highest commercial quality and used without further purification, unless otherwise stated. Reactions were monitored by thin-layer chromatography (TLC) carried out on $0.25 \mathrm{~mm}$ E. Merck silica gel plates (60F-254) using UV light as visualizing agent and an ethanolic solution of $p$-anisaldehyde and heat as a developing agent. E. Merck silica gel (60, particle size $0.040-0.063 \mathrm{~mm}$ ) was used for flash column chromatography. Preparative thinlayer chromatography separations were carried out on 0.25 or $0.50 \mathrm{~mm}$ E. Merck silica gel plates (60F-254). NMR spectra were recorded on Bruker DRX-600 or DRX-500 instruments and calibrated using residual undeuterated solvent as an internal reference. The following 
abbreviations were used to explain the multiplicities: $\mathrm{s}=$ singlet, $\mathrm{d}=$ doublet, $\mathrm{t}=$ triplet, $\mathrm{q}=$ quartet, $\mathrm{m}=$ multiplet, pent $=$ pentet, hex $=$ hexet, $\mathrm{br}=$ broad. IR spectra were recorded on a Perkin-Elmer Spectrum 100 FT-IR spectrometer. LC/MS data were recorded on an Agilent 1100 series LC system coupled to an ESI Agilent MSD. Melting points (m.p.) are uncorrected and were recorded on a Thomas Hoover Uni-Melt melting point apparatus. High-resolution mass spectra (HRMS) were recorded on an Agilent ESI TOF (time of flight) mass spectrometer at $4000 \mathrm{~V}$ emitter voltage.

\section{Napthoquinone 5}

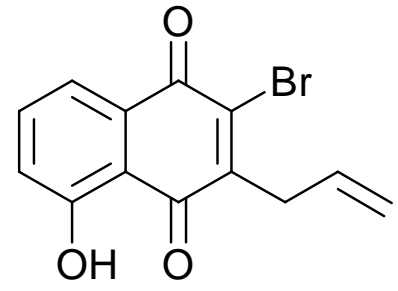

5

To a solution of bromo-nathoquinone 4 ( $4.0 \mathrm{~g}, 15.8 \mathrm{mmol}, 1.0$ equiv) in degassed $\mathrm{MeCN}(120 \mathrm{~mL})$ at $25{ }^{\circ} \mathrm{C}$, was added $\mathrm{AgNO}_{3}(0.80 \mathrm{~g}, 4.74$ mmol, 0.3 equiv) and vinylacetic acid $(2.0 \mathrm{~mL}, 21.1 \mathrm{mmol}, 1.3$ equiv) and the reaction mixture was then heated to $65^{\circ} \mathrm{C}$. $\left(\mathrm{NH}_{4}\right)_{2} \mathrm{~S}_{2} \mathrm{O}_{8}(7.2 \mathrm{~g}$, 31.6 mmol, 2.0 equiv) in degassed $\mathrm{H}_{2} \mathrm{O}(60 \mathrm{~mL})$ was then added dropwise to the reaction mixture at $65^{\circ} \mathrm{C}$ over $30 \mathrm{~min}$. After $3 \mathrm{~h}$ (starting material consumed by TLC), the reaction mixture was cooled to $25{ }^{\circ} \mathrm{C}$, and the reaction mixture was extracted with EtOAc $(3 \times 150 \mathrm{~mL})$, and the combined organic layers were washed with brine $(150 \mathrm{~mL})$, dried $\left(\mathrm{MgSO}_{4}\right)$, and concentrated. Flash column chromatography (silica gel, hexanes:EtOAc 6:1) provided bromo-napthoquinone $5(3.5 \mathrm{~g}, 11.9 \mathrm{mmol}, 75 \%$ yield $)$ as a red-brown foam. $5: \mathrm{R}_{f}=$ 0.59 (silica gel, hexanes:EtOAc 4:1); IR (film) $v_{\max } 3081,1672,1631,1588,1454,1426,1365$, $1269,1255,1211,1166,1131,1095,1057,998,921,834,799,777,741,709 \mathrm{~cm}^{-1} ;{ }^{1} \mathrm{H}$ NMR $\left(600 \mathrm{MHz}, \mathrm{CDCl}_{3}\right): \delta=7.70(\mathrm{~d}, J=7.8 \mathrm{~Hz}, 1 \mathrm{H}), 7.61(\mathrm{t}, J=7.8 \mathrm{~Hz}, 1 \mathrm{H}), 7.28(\mathrm{~d}, J=7.8 \mathrm{~Hz}, 1$ H), $5.85(\mathrm{ddt}, J=16.8,10.2,6.6 \mathrm{~Hz}, 1 \mathrm{H}), 5.26(\mathrm{~d}, J=16.8 \mathrm{~Hz}, 1 \mathrm{H}), 5.16(\mathrm{~d}, J=10.2 \mathrm{~Hz}, 1 \mathrm{H})$, $3.61 \mathrm{ppm}(\mathrm{d}, J=6.6 \mathrm{~Hz}, 2 \mathrm{H}) ;{ }^{13} \mathrm{C}$ NMR $\left(150 \mathrm{MHz}, \mathrm{CDCl}_{3}\right): \delta=186.5,177.1,161.8,148.9$, 140.2, 136.3, 131.1, 131.0, 124.8, 120.6, 118.4, 114.4, 34.8 ppm; HRMS (ESI-TOF): calcd for $\mathrm{C}_{13} \mathrm{H}_{9} \mathrm{BrO}_{3}\left[\mathrm{M}+\mathrm{H}^{+}\right]: 292.9808$, found 292.9801. 


\section{Benzyl-protected bromo-napthoquinone 5a}<smiles>C=CCC1=C(Br)C(=O)c2cccc(OCc3ccccc3)c2C1=O</smiles>

$5 \mathbf{a}$

To a solution of bromo-napthoquinone 5 (340 mg, $1.16 \mathrm{mmol}, 1.0$ equiv) in $\mathrm{CH}_{2} \mathrm{Cl}_{2}(12 \mathrm{~mL})$ at $25{ }^{\circ} \mathrm{C}$ was added $\mathrm{BnBr}(0.28 \mathrm{~mL}, 2.32$ mmol, 2.0 equiv), and freshly prepared $\mathrm{Ag}_{2} \mathrm{O}(270 \mathrm{mg}, 1.16 \mathrm{mmol}, 1.0$ equiv). The reaction mixture was stirred at $25{ }^{\circ} \mathrm{C}$ for $18 \mathrm{~h}$, then filtered through a plug of Celite and concentrated. Flash column chromatography (silica gel, hexanes:EtOAc 4:1) provided benzyl-protected bromonapthoquinone $5 \mathbf{a}\left(410 \mathrm{mg}, 1.10 \mathrm{mmol}, 92 \%\right.$ yield) as a colorless foam. 5a: $\mathrm{R}_{f}=0.53$ (silica gel, hexanes:EtOAc 2:1); IR (film) $v_{\max }$ 3065, 3017, 2921, 2860, 1670, 1604, 1583, 1498, 1447 , $1383,1273,1257,1220,1161,1096,1050,972,913,828,772,738,696 \mathrm{~cm}^{-1} ;{ }^{1} \mathrm{H}$ NMR $(600$ $\left.\mathrm{MHz}, \mathrm{CDCl}_{3}\right): \delta=7.82(\mathrm{dd}, J=7.2,0.6 \mathrm{~Hz}, 1 \mathrm{H}), 7.60(\mathrm{dd}, J=7.2,0.6 \mathrm{~Hz}, 1 \mathrm{H}), 7.55(\mathrm{~d}, J=7.2$ Hz, 2 H), 7.42 (t, $J=7.2 \mathrm{~Hz}, 2 \mathrm{H}), 7.33(\mathrm{t}, J=7.2 \mathrm{~Hz}, 2 \mathrm{H}), 5.88(\mathrm{ddt}, J=16.8,10.2,6.6 \mathrm{~Hz}, 1$ H), $5.31(\mathrm{~s}, 2 \mathrm{H}), 5.27$ (dd, $J=16.8,1.2 \mathrm{~Hz}, 1 \mathrm{H}), 5.14(\mathrm{dd}, J=10.2,1.2 \mathrm{~Hz}, 1 \mathrm{H}), 3.63 \mathrm{ppm}(\mathrm{dt}$, $J=6.6,1.2 \mathrm{~Hz}, 2 \mathrm{H}) ;{ }^{13} \mathrm{C} \mathrm{NMR}\left(150 \mathrm{MHz}, \mathrm{CDCl}_{3}\right): \delta=180.0,178.1,158.9,150.7,136.6,135.9$, 134.7, 133.3, 131.8, 128.7, 128.1, 126.7, 120.6, 119.9, 119.8, 118.1, 71.0, 35.7 ppm; HRMS (ESI-TOF): calcd for $\mathrm{C}_{20} \mathrm{H}_{16} \mathrm{BrO}_{3}\left[\mathrm{M}+\mathrm{H}^{+}\right]$: 383.0277, found 383.0282.

\section{Methyl-protected hydroquinone 6}

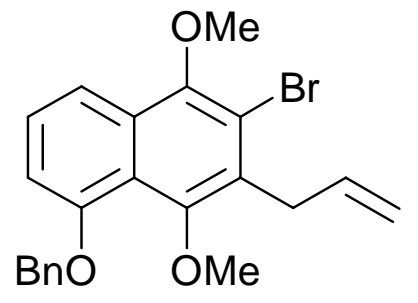

6

To a solution of benzyl-protected bromo-napthoquinone 5a $(200 \mathrm{mg}$, $0.52 \mathrm{mmol}, 1.0$ equiv) in EtOAc $(1 \mathrm{~mL}), \mathrm{Et}_{2} \mathrm{O}(10 \mathrm{~mL})$, and $\mathrm{H}_{2} \mathrm{O}(10$ $\mathrm{mL})$ at $25^{\circ} \mathrm{C}$ was added $\mathrm{Na}_{2} \mathrm{~S}_{2} \mathrm{O}_{4}(454 \mathrm{~g}, 2.61 \mathrm{mmol}, 5.0$ equiv) and the reaction mixture was stirred vigorously for $30 \mathrm{~min}$. Upon consumption of starting material (TLC), the biphasic reaction mixture was extracted with EtOAc $(3 \times 10 \mathrm{~mL})$, the combined organic layers washed with brine $(15 \mathrm{~mL})$, dried $\left(\mathrm{MgSO}_{4}\right)$, and concentrated. The resulting oil was then dissolved in DMF $(5 \mathrm{~mL})$ and cooled to $-15^{\circ} \mathrm{C}$. $\mathrm{NaH}$ (44 mg, $1.10 \mathrm{mmol}, 2.1$ equiv) was added portionwise to the reaction mixture, and then $\mathrm{MeI}(71 \mu \mathrm{L}, 1.15 \mathrm{mmol}, 2.2$ equiv) was added dropwise over $5 \mathrm{~min}$, and the reaction 
mixture was stirred at $-15{ }^{\circ} \mathrm{C}$ for $1 \mathrm{~h}$. The reaction mixture was then quenched with sat. aqueous $\mathrm{NH}_{4} \mathrm{Cl}(10 \mathrm{~mL})$ and the biphasic reaction mixture was extracted with EtOAc $(3 \times 10 \mathrm{~mL})$, and the combined organic layers washed with brine $(25 \mathrm{~mL})$, dried $\left(\mathrm{MgSO}_{4}\right)$, and concentrated. Flash column chromatography (silica gel, hexanes:EtOAc 8:1) gave methyl-protected hydroquinone 6 (177 mg, $0.43 \mathrm{mmol}, 82 \%$ yield) as a colorless foam. 6: $\mathrm{R}_{f}=0.64$ (silica gel, hexanes:EtOAc 4:1); IR (film) $v_{\max }$ 3068, 2931, 2840, 1637, 1611, 1561, 1497, 1453, 1440, $1385,1365,1324,1264,1160,1120,1081,1051,1030,1015,976,911,844,812,793,750,697$ $\mathrm{cm}^{-1} ;{ }^{1} \mathrm{H}$ NMR $\left(600 \mathrm{MHz}, \mathrm{CDCl}_{3}\right): \delta=7.76(\mathrm{~d}, J=8.4 \mathrm{~Hz}, 1 \mathrm{H}), 7.57(\mathrm{~d}, J=7.2 \mathrm{~Hz}, 2 \mathrm{H}), 7.43$ (t, $J=7.2 \mathrm{~Hz}, 2 \mathrm{H}), 7.41(\mathrm{t}, J=8.4 \mathrm{~Hz}, 1 \mathrm{H}), 7.37$ (t, $J=7.2 \mathrm{~Hz}, 1 \mathrm{H}), 7.00$ (d, $J=8.4 \mathrm{~Hz}, 1 \mathrm{H})$, $6.09(\mathrm{ddt}, J=16.8,10.2,5.4 \mathrm{~Hz}, 1 \mathrm{H}), 5.23(\mathrm{~s}, 2 \mathrm{H}), 5.10(\mathrm{~d}, J=10.2 \mathrm{~Hz}, 1 \mathrm{H}), 5.05(\mathrm{~d}, J=16.8$

$\mathrm{Hz}, 1 \mathrm{H}), 3.96(\mathrm{~s}, 3 \mathrm{H}), 3.82(\mathrm{~d}, J=5.4 \mathrm{~Hz}, 2 \mathrm{H}), 3.71 \mathrm{ppm}(\mathrm{s}, 3 \mathrm{H}) ;{ }^{13} \mathrm{C} \mathrm{NMR}(150 \mathrm{MHz}$, $\left.\mathrm{CDCl}_{3}\right): \delta=155.2,151.4,149.6,136.8,136.2,130.5,129.8,128.5,127.9,127.6,126.6,120.4$ 117.5, 115.7, 115.4, 108.7, 71.6, 63.1, 61.1, 34.3 ppm; HRMS (ESI-TOF): calcd for $\mathrm{C}_{22} \mathrm{H}_{22} \mathrm{BrO}_{3}$ $\left[\mathrm{M}+\mathrm{H}^{+}\right]:$413.0747, found 413.0737.

\section{Double bond migrated napthoquinone 6a}<smiles>C/C=C/c1c(Br)c(OC)c2cccc(OCc3ccccc3)c2c1OC</smiles>

6a

To a cold $\left(0^{\circ} \mathrm{C}\right)$ solution of methyl-protected hydroquinone 6 (140 mg, 0.34 mmol, 1.0 equiv) in THF (4 mL) was added $t$-BuOK (680 $\mu \mathrm{L}, 1.0 \mathrm{M}$ in $\mathrm{THF}, 0.68 \mathrm{mmol}, 2.0$ equiv) and the reaction mixture was stirred for $2 \mathrm{~h}$ at $0{ }^{\circ} \mathrm{C}$. The reaction mixture was then quenched with $\mathrm{H}_{2} \mathrm{O}(5 \mathrm{~mL})$ and the biphasic reaction mixture was extracted with EtOAc $(3 \times 5 \mathrm{~mL})$. The combined organic layers were dried $\left(\mathrm{MgSO}_{4}\right)$, concentrated, and double bond migrated napthoquinone 6a (137 $\mathrm{mg}, 0.33 \mathrm{mmol}, 98 \%$ yield) was used without further purification. 6a: $\mathrm{R}_{f}=0.59$ (silica gel, hexanes:EtOAc 4:1); IR (film) $v_{\max } 3027,2929$, $2835,1609,1553,1498,1449,1380,1361,1324,1262,1205,1176,1159,1125,1080,1053$, 1030, 980, 912, 781, 760, $697 \mathrm{~cm}^{-1} ;{ }^{1} \mathrm{H}$ NMR (600 MHz, $\left.\mathrm{CDCl}_{3}\right): \delta=7.72(\mathrm{~d}, J=8.4 \mathrm{~Hz}, 1 \mathrm{H})$, $7.57(\mathrm{~d}, J=7.8 \mathrm{~Hz}, 2 \mathrm{H}), 7.42(\mathrm{t}, J=7.8 \mathrm{~Hz}, 2 \mathrm{H}), 7.38(\mathrm{t}, J=8.4 \mathrm{~Hz}, 1 \mathrm{H}), 7.36(\mathrm{t}, J=7.8 \mathrm{~Hz}, 1$ 
H), $6.98(\mathrm{~d}, J=8.4 \mathrm{~Hz}, 1 \mathrm{H}), 6.55(\mathrm{~d}, J=15.6 \mathrm{~Hz}, 1 \mathrm{H}), 6.42(\mathrm{dq}, J=15.6,6.6 \mathrm{~Hz}, 1 \mathrm{H}), 5.22$, (s, $2 \mathrm{H}), 3.95$ (s, $3 \mathrm{H}), 3.61(\mathrm{~s}, 3 \mathrm{H}), 1.99 \mathrm{ppm}(\mathrm{d}, J=6.6 \mathrm{~Hz}, 3 \mathrm{H}) ;{ }^{13} \mathrm{C} \mathrm{NMR}\left(150 \mathrm{MHz}, \mathrm{CDCl}_{3}\right): \delta$ $=155.6,151.3,149.5,136.9,133.1,130.2,129.0,128.5,127.9,127.6,126.7,125.6,120.7$, 116.3, 115.3, 108.7, 71.5, 61.3, 61.0, 19.3 ppm; HRMS (ESI-TOF): calcd for $\mathrm{C}_{22} \mathrm{H}_{22} \mathrm{BrO}_{3}$ $\left[\mathrm{M}+\mathrm{H}^{+}\right]:$413.0747, found 413.0728.

\section{Bromo-aldehyde coupling partner 2}

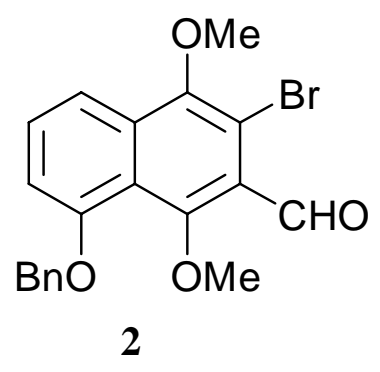

To a solution of double bond migrated napthoquinone 6a (130 mg, 0.32 mmol, 1.0 equiv) in THF $(2 \mathrm{~mL})$ and $\mathrm{H}_{2} \mathrm{O}(1 \mathrm{~mL})$ at $25^{\circ} \mathrm{C}$ was added $\mathrm{OsO}_{4}\left(0.8 \mathrm{mg}, 0.0032 \mathrm{mmol}, 0.01\right.$ equiv) and $\mathrm{NaIO}_{4}(162 \mathrm{mg}, 0.76 \mathrm{mmol}$, 2.4 equiv). The reaction mixture was then heated to $70^{\circ} \mathrm{C}$ and stirred for $18 \mathrm{~h}$. The reaction mixture was then quenched with sat. aqueous $\mathrm{Na}_{2} \mathrm{~S}_{2} \mathrm{O}_{3}$ $(5 \mathrm{~mL})$ and stirred vigorously for $30 \mathrm{~min}$. The biphasic reaction mixture was then extracted with EtOAc $(3 \times 10 \mathrm{~mL})$ and the combined organic layers were washed with brine $(15 \mathrm{~mL})$, dried $\left(\mathrm{MgSO}_{4}\right)$, and concentrated. Flash column chromatography (silica gel, hexanes:EtOAc 6:1) provided bromo-aldehyde coupling partner 2 (108 $\mathrm{mg}, 0.27 \mathrm{mmol}, 84 \%$ yield) as a colorless oil. 2: $\mathrm{R}_{f}=0.30$ (silica gel, hexanes:EtOAc 4:1); IR (film) $v_{\max } 2931,2844,1694,1607,1550,1497$, $1452,1440,1365,1317,1265,1240,1207,1178,1125,1081,1049,1013,970,930,843,813$, 756, 740, $697 \mathrm{~cm}^{-1} ;{ }^{1} \mathrm{H} \mathrm{NMR}\left(600 \mathrm{MHz}, \mathrm{CDCl}_{3}\right): \delta=10.5(\mathrm{~s}, 1 \mathrm{H}), 7.76(\mathrm{~d}, J=8.4 \mathrm{~Hz}, 1 \mathrm{H})$, $7.58(\mathrm{t}, J=8.4 \mathrm{~Hz}, 1 \mathrm{H}), 7.55(\mathrm{~d}, J=7.8 \mathrm{~Hz}, 2 \mathrm{H}), 7.43(\mathrm{t}, J=7.8 \mathrm{~Hz}, 2 \mathrm{H}), 7.38(\mathrm{t}, J=7.8 \mathrm{~Hz}, 1$ H), $7.07(\mathrm{~d}, J=8.4 \mathrm{~Hz}, 1 \mathrm{H}), 5.24(\mathrm{~s}, 2 \mathrm{H}), 3.97$ (s, $3 \mathrm{H}), 3.78 \mathrm{ppm}(\mathrm{s}, 3 \mathrm{H}) ;{ }^{13} \mathrm{C}$ NMR $(150$ $\left.\mathrm{MHz}, \mathrm{CDCl}_{3}\right): \delta=190.7,158.9,156.8,150.4,136.1,133.9,130.3,128.6,128.2,127.6,125.1$, 120.1, 115.4, 111.3, 109.1, 71.5, 65.3, 61.2 ppm; HRMS (ESI-TOF): calcd for $\mathrm{C}_{20} \mathrm{H}_{18} \mathrm{BrO}_{4}$ $\left[\mathrm{M}+\mathrm{H}^{+}\right]:$401.0383, found 401.0379. 


\section{Enone 7}<smiles>O=C1C=CCCC1</smiles>

TBSO

7

7: $80 \%$ ee, obtained according to ref 7. $\mathrm{R}_{f}=0.48$ (silica gel, hexanes:EtOAc 4:1); $[\alpha]_{\mathrm{D}}^{32}=+92.2\left(\mathrm{CDCl}_{3}, c=2.33\right) ;$ IR (film) $v_{\max } 2954,2930,2887,2857,1688,1472$, $1463,1381,1250,1200,1132,1096,1066,997,984,971,956,857,834,774,730$, $667 \mathrm{~cm}^{-1} ;{ }^{1} \mathrm{H} \mathrm{NMR}\left(500 \mathrm{MHz}, \mathrm{CDCl}_{3}\right): \delta=6.80(\mathrm{ddd}, J=10.5,2.0,1.5 \mathrm{~Hz}, 1 \mathrm{H})$, $5.89(\mathrm{ddd}, J=10.5,1.5,1.0 \mathrm{~Hz}, 1 \mathrm{H}), 4.50$ (dddd, $J=9.0,4.5,2.5,2.0 \mathrm{~Hz}, 1 \mathrm{H}), 2.54$ $(\mathrm{dt}, J=17.0,4.5 \mathrm{~Hz}, 1 \mathrm{H}), 2.31(\mathrm{ddd}, J=17.5,13.0,4.5 \mathrm{~Hz}, 1 \mathrm{H}), 2.22-2.14(\mathrm{~m}, 1 \mathrm{H}), 2.01-1.92$ $(\mathrm{m}, 1 \mathrm{H}), 0.88(\mathrm{~s}, 9 \mathrm{H}), 0.10(\mathrm{~s}, 3 \mathrm{H}), 0.09 \mathrm{ppm}(\mathrm{s}, 3 \mathrm{H}) ;{ }^{13} \mathrm{C} \mathrm{NMR}\left(125 \mathrm{MHz}, \mathrm{CDCl}_{3}\right): \delta=198.6$, 153.7, 128.6, 66.9, 35.4, 32.9, 25.7, 18.0, -4.7, -4.8 ppm; HRMS (ESI-TOF): calcd for $\mathrm{C}_{12} \mathrm{H}_{23} \mathrm{O}_{2} \mathrm{Si}\left[\mathrm{M}+\mathrm{H}^{+}\right]: 227.1462$, found 227.1470.

\section{Methylated enone 8}

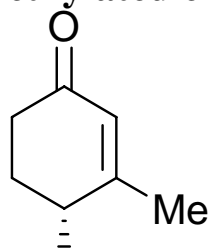

TBSŌ

8

To a solution of $\mathrm{MeMgBr}(18.0 \mathrm{~mL}, 3.0 \mathrm{M}$ in THF, $53.9 \mathrm{mmol}, 2.0$ equiv) in THF (80 mL) at $-78{ }^{\circ} \mathrm{C}$ was added $\mathrm{CuBr} \cdot \mathrm{DMS}(553 \mathrm{mg}, 2.7 \mathrm{mmol}, 0.1$ equiv) in HMPA (11 mL) dropwise over $10 \mathrm{~min}$, and the reaction mixture was stirred at $78^{\circ} \mathrm{C}$ for $30 \mathrm{~min}$. A solution of enone $7(6.1 \mathrm{~g}, 26.9 \mathrm{mmol}, 1.0$ equiv) in THF (30 $\mathrm{mL})$ and $\mathrm{TMSCl}(8.6 \mathrm{~mL}, 67.3 \mathrm{mmol}, 2.5$ equiv) was then added dropwise to the reaction mixture over $45 \mathrm{~min}$, and the reaction mixture was stirred at $-78{ }^{\circ} \mathrm{C}$ for $30 \mathrm{~min}$ and then warmed to $25^{\circ} \mathrm{C}$ over $30 \mathrm{~min}$. The reaction mixture was then quenched with $\mathrm{Et}_{3} \mathrm{~N}$ (13 $\mathrm{mL})$, hexanes $(120 \mathrm{~mL})$, and $\mathrm{pH} 7$ buffer $(120 \mathrm{~mL})$, and the resulting slurry was filtered through a plug of Celite. The biphasic filtrate was then extracted with EtOAc $(3 \times 100 \mathrm{~mL})$, the combined organic layers were washed with brine $(2 \times 100 \mathrm{~mL})$, and then concentrated, without further drying. The resulting oil was dissolved in DMSO $(250 \mathrm{~mL})$, and $\mathrm{Pd}(\mathrm{OAc})_{2}(1.8 \mathrm{~g}, 2.7$ mmol, 0.1 equiv) was added in one portion to the reaction mixture at $25^{\circ} \mathrm{C}$, under an oxygen atmosphere (balloon, $1 \mathrm{~atm}$ ). The reaction mixture was stirred at $25{ }^{\circ} \mathrm{C}$ for $18 \mathrm{~h}$, and then quenched with $\mathrm{H}_{2} \mathrm{O}(250 \mathrm{~mL})$ and the biphasic reaction mixture was extracted with EtOAc $(3 \times$ $200 \mathrm{~mL})$. The combined organic layers were dried $\left(\mathrm{MgSO}_{4}\right)$, and concentrated. Flash column 
chromatography (silica gel, hexanes:EtOAc 10:1) afforded methylated enone 8 (5.8 g, 24.1 mmol, 90\% yield over two steps) as a colorless oil. 8: $\mathrm{R}_{f}=0.44$ (silica gel, hexanes:EtOAc 4:1); $[\alpha]_{\mathrm{D}}^{32}=+26.0\left(\mathrm{CDCl}_{3}, c=1.53\right)$; IR (film) $v_{\max } 2954,2929,2887,2857,1673,1472,1463,1440$, 1374, 1360, 1324, 1252, 1195, 1096, 1098, 1066, 1004, 989, 972, 895, 879, 855, 835, 803, 774, $671 \mathrm{~cm}^{-1} ;{ }^{1} \mathrm{H}$ NMR $\left(500 \mathrm{MHz}, \mathrm{CDCl}_{3}\right): \delta=5.75(\mathrm{~d}, J=1.0 \mathrm{~Hz}, 1 \mathrm{H}), 4.30(\mathrm{dd}, J=8.5,4.5 \mathrm{~Hz}, 1$ H), $2.48(\mathrm{dt}, J=17.0,5.0 \mathrm{~Hz}, 1 \mathrm{H}), 2.26(\mathrm{ddd}, J=17.0,12.0,5.0 \mathrm{~Hz}, 1 \mathrm{H}), 2.15-2.06(\mathrm{~m}, 1 \mathrm{H})$, 1.97-1.88 (m, $1 \mathrm{H}), 1.92(\mathrm{~s}, 3 \mathrm{H}), 0.86(\mathrm{~s}, 9 \mathrm{H}), 0.08(\mathrm{~s}, 3 \mathrm{H}), 0.07 \mathrm{ppm}(\mathrm{s}, 3 \mathrm{H}) ;{ }^{13} \mathrm{C}$ NMR $(125$ $\left.\mathrm{MHz}_{\mathrm{CDCl}}\right): \delta=198.4,164.0,126.4,69.5,34.9,32.4,25.6,20.9,17.9,-4.5,-5.1 \mathrm{ppm}$; HRMS (ESI-TOF): calcd for $\mathrm{C}_{13} \mathrm{H}_{25} \mathrm{O}_{2} \mathrm{Si}\left[\mathrm{M}+\mathrm{H}^{+}\right]$: 241.1618, found 241.1622.

\section{Diol 9}

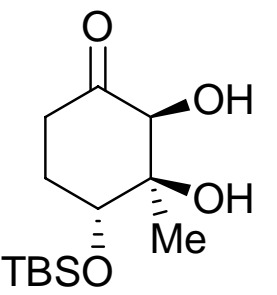

To a solution of methylated enone 8 (5.8 g, $24.1 \mathrm{mmol}, 1.0$ equiv) in acetone (45 mL) and $\mathrm{H}_{2} \mathrm{O}(4.5 \mathrm{~mL})$ at $25{ }^{\circ} \mathrm{C}$, was added NMO (4.2 g, $36.2 \mathrm{mmol}, 1.5$ equiv), and $\mathrm{OsO}_{4}(125 \mathrm{mg}, 0.48 \mathrm{mmol}, 0.02$ equiv), and the reaction mixture 9 was stirred at $25^{\circ} \mathrm{C}$ for $45 \mathrm{~min}$. The reaction mixture was then quenched with sat. aqueous $\mathrm{Na}_{2} \mathrm{~S}_{2} \mathrm{O}_{4}(50 \mathrm{~mL})$, and the biphasic reaction mixture was stirred at $25^{\circ} \mathrm{C}$ for $30 \mathrm{~min}$, and then extracted with EtOAc $(3 \times 50 \mathrm{~mL})$. The combined organic layers were then washed with brine $(100 \mathrm{~mL})$, dried $\left(\mathrm{MgSO}_{4}\right)$, and concentrated. Flash column chromatography (silica gel, hexanes:EtOAc 4:1) afforded diol 9 (5.0 g, $18.2 \mathrm{mmol}, 76 \%$ yield) as a colorless oil after three recrystallizations (hexanes/EtOAc), which removed the minor wrong enantiomer. 9: $\mathrm{R}_{f}=0.16$ (silica gel, hexanes:EtOAc 4:1); $[\alpha]_{\mathrm{D}}{ }^{32}=-45.6\left(\mathrm{CDCl}_{3}, c=1.58\right)$; IR (film) $v_{\max } 3468,2951,2931,2858,2886,1722,1463,1390,1361,1254,1086,1041,990,963$, 939, 885, 853, 837, 810, 776, $681 \mathrm{~cm}^{-1} ;{ }^{1} \mathrm{H}$ NMR (600 MHz, $\left.\mathrm{CDCl}_{3}\right): \delta=4.27(\mathrm{~s}, 1 \mathrm{H}), 3.89(\mathrm{~s}, 1$ H), $3.69(\mathrm{~s}, 1 \mathrm{H}), 2.74(\mathrm{td}, J=13.8,6.6 \mathrm{~Hz}, 1 \mathrm{H}), 2.38-2.34(\mathrm{~m}, 2 \mathrm{H}), 2.32-2.26$ (m, $1 \mathrm{H}), 1.90-$ $1.85(\mathrm{~m}, 1 \mathrm{H}), 1.40(\mathrm{~s}, 3 \mathrm{H}), 0.92(\mathrm{~s}, 9 \mathrm{H}), 0.13(\mathrm{~s}, 3 \mathrm{H}), 0.12 \mathrm{ppm}(\mathrm{s}, 3 \mathrm{H}) ;{ }^{13} \mathrm{C} \mathrm{NMR}(150 \mathrm{MHz}$, $\left.\mathrm{CDCl}_{3}\right): \delta=210.6,79.6,77.5,73.4,33.2,29.2,25.7,23.7,17.9,-4.5,-5.0$ ppm; HRMS (ESITOF): calcd for $\mathrm{C}_{13} \mathrm{H}_{27} \mathrm{O}_{4} \mathrm{Si}\left[\mathrm{M}+\mathrm{H}^{+}\right]$: 275.1673, found 275.1675. 


\section{Acetonide 10

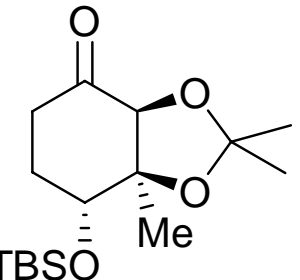

10

To a solution of diol 9 (5.0 g, $18.2 \mathrm{mmol}, 1.0$ equiv) in THF (625 mL) at 25 ${ }^{\circ} \mathrm{C}$ was added 2-methoxy-propene (17.4 mL, $182 \mathrm{mmol}, 10.0$ equiv), and CSA ( $85 \mathrm{mg}, 0.37 \mathrm{mmol}, 0.02$ equiv), and the reaction mixture was stirred at $25^{\circ} \mathrm{C}$ for $18 \mathrm{~h}$. The reaction mixture was then quenched with $\mathrm{H}_{2} \mathrm{O}(500 \mathrm{~mL})$ and the biphasic reaction mixture was extracted with EtOAc $(3 \times 500 \mathrm{~mL})$, the combined organic layers were dried $\left(\mathrm{MgSO}_{4}\right)$, and concentrated. Flash column chromatography (silica gel, hexanes:EtOAc 20:1) provided acetonide 10 (5.4 g, $17.2 \mathrm{mmol}, 95 \%$ yield) as a colorless oil. 10: $\mathrm{R}_{f}=0.54$ (silica gel, hexanes:EtOAc 4:1); $[\alpha]_{\mathrm{D}}{ }^{32}=-36.8\left(\mathrm{CDCl}_{3}, c\right.$ $=1.83$ ); IR (film) $v_{\max } 2982,2932,2888,2858,1729,1472,1463,1408,1374,1330,1250,1210$, 1177, 1137, 1087, 1063, 1042, 996, 969, 939, 912, 892, 856, 835, 809, 774, 750, 680, $656 \mathrm{~cm}^{-1}$; ${ }^{1} \mathrm{H}$ NMR $\left(600 \mathrm{MHz}, \mathrm{CDCl}_{3}\right): \delta=3.93(\mathrm{~s}, 1 \mathrm{H}), 3.72(\mathrm{~d}, J=5.4 \mathrm{~Hz}, 1 \mathrm{H}), 2.52-2.36(\mathrm{~m}, 3 \mathrm{H})$, $1.84(\mathrm{~m}, 1 \mathrm{H}), 1.45(\mathrm{~s}, 3 \mathrm{H}), 1.40(\mathrm{~s}, 3 \mathrm{H}), 1.39$ (s, $3 \mathrm{H}), 0.85(\mathrm{~s}, 9 \mathrm{H}), 0.06 \mathrm{ppm}(\mathrm{s}, 6 \mathrm{H}) ;{ }^{13} \mathrm{C}$ NMR (150 MHz, $\left.\mathrm{CDCl}_{3}\right): \delta=207.4,111.0,87.0,84.0,71.6,31.7,27.1,26.5,25.6,25.4,23.8$, 17.9, 17.9, -4.7, -5.0 ppm; HRMS (ESI-TOF): calcd for $\mathrm{C}_{16} \mathrm{H}_{31} \mathrm{O}_{4} \mathrm{Si}\left[\mathrm{M}+\mathrm{H}^{+}\right]$: 315.1986 , found 315.1987.

\section{Enone 11}

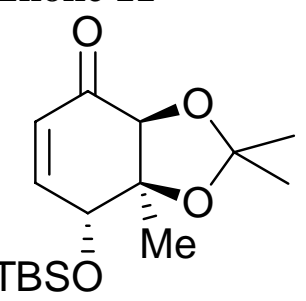

11

To a cold $\left(0{ }^{\circ} \mathrm{C}\right)$ solution of acetonide $10(4.4 \mathrm{~g}, 14.0 \mathrm{mmol}, 1.0$ equiv) in THF $(140 \mathrm{~mL})$ was added LiHMDS $(42.0 \mathrm{~mL}, 1.0 \mathrm{M}$ in THF, $42.0 \mathrm{mmol}$, 3.0 equiv) dropwise over $10 \mathrm{~min}$, followed by TMSCl $(5.4 \mathrm{~mL}, 42.0 \mathrm{mmol}$, 3.0 equiv) dropwise addition over $5 \mathrm{~min}$. The reaction mixture was stirred for $20 \mathrm{~min}$ at $0{ }^{\circ} \mathrm{C}$, and then warmed to $25{ }^{\circ} \mathrm{C}$ over $10 \mathrm{~min}$. Upon disappearance of the starting material (TLC), the reaction mixture was quenched with $\mathrm{H}_{2} \mathrm{O}$ (100 $\mathrm{mL})$, and the biphasic reaction mixture was extracted with EtOAc $(3 \times 100 \mathrm{~mL})$, and the combined organic layers were washed with brine $(2 \times 100 \mathrm{~mL})$, and concentrated without further 
drying. The residual oil was then dissolved in DMSO $(140 \mathrm{~mL})$, and $\mathrm{Pd}(\mathrm{OAc})_{2}(940 \mathrm{mg}, 1.4$ mmol, 0.1 equiv) was added in one portion, and the reaction mixture was stirred under an oxygen atmosphere (balloon, $1 \mathrm{~atm}$ ) at $25^{\circ} \mathrm{C}$ for $18 \mathrm{~h}$. The reaction mixture was then quenched with $\mathrm{H}_{2} \mathrm{O}(150 \mathrm{~mL})$, and the biphasic reaction mixture was extracted with EtOAc $(3 \times 150 \mathrm{~mL})$, the combined organic layers were dried $\left(\mathrm{MgSO}_{4}\right)$, and concentrated. Flash column chromatography (silica gel, hexanes:EtOAc 10:1) gave enone 11 (3.7 g, 11.8 mmol, 84\% yield over two steps). 11: $\mathrm{R}_{f}=0.57$ (silica gel, hexanes:EtOAc 4:1); $[\alpha]_{\mathrm{D}}^{32}=-101.4\left(\mathrm{CDCl}_{3}, c=2.0\right)$; IR (film) $v_{\max }$ 2987, 2955, 2932, 2881, 2858, 1691, 1472, 1374, 1339, 1250, 1216, 1194, 1175, 1134, 1119, 1095, 1053, 1019, 992, 940, 889, 850, 836, 778, $696 \mathrm{~cm}^{-1} ;{ }^{1} \mathrm{H} \mathrm{NMR}\left(600 \mathrm{MHz}, \mathrm{CDCl}_{3}\right): \delta=6.77$ $(\mathrm{dd}, J=10.2,1.8 \mathrm{~Hz}, 1 \mathrm{H}), 6.05(\mathrm{dd}, J=10.2,2.1 \mathrm{~Hz}, 1 \mathrm{H}), 4.58(\mathrm{bt}, J=2.1 \mathrm{~Hz}, 1 \mathrm{H}), 4.10$ (s, 1 $\mathrm{H}), 1.49(\mathrm{~s}, 3 \mathrm{H}), 1.44(\mathrm{~s}, 3 \mathrm{H}), 1.32(\mathrm{~s}, 3 \mathrm{H}), 0.93(\mathrm{~s}, 9 \mathrm{H}), 0.16(\mathrm{~s}, 3 \mathrm{H}), 0.12 \mathrm{ppm}(\mathrm{s}, 3 \mathrm{H}) ;{ }^{13} \mathrm{C}$ NMR $\left(150 \mathrm{MHz}, \mathrm{CDCl}_{3}\right): \delta=192.6,154.6,126.9,110.4,85.4,81.0,72.6,27.8,26.9,25.7,18.7$, 18.0, -4.5, -4.9 ppm; HRMS (ESI-TOF): calcd for $\mathrm{C}_{16} \mathrm{H}_{29} \mathrm{O}_{4} \mathrm{Si}\left[\mathrm{M}+\mathrm{H}^{+}\right]$: 313.1830 , found 313.1822 .

\section{Iodide coupling partner 3}

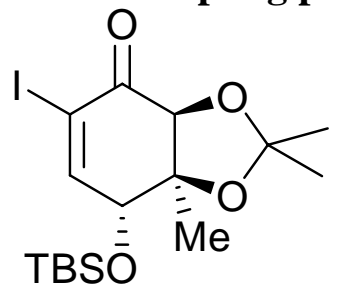

3

To a solution of enone 11 (1.4 g, $4.9 \mathrm{mmol}, 1.0$ equiv) in $\mathrm{CH}_{2} \mathrm{Cl}_{2}$ (7 mL) and pyridine $(14 \mathrm{~mL})$ at $25{ }^{\circ} \mathrm{C}$ was added $\mathrm{I}_{2}(3.4 \mathrm{~g}, 13.4 \mathrm{mmol}, 3.0$ equiv) portionwise. After $30 \mathrm{~min}$ (starting material consumed by TLC), the reaction mixture was quenched with $\mathrm{H}_{2} \mathrm{O}(50 \mathrm{~mL})$ and $\mathrm{Na}_{2} \mathrm{~S}_{2} \mathrm{O}_{3}(5 \mathrm{~mL})$, and the biphasic reaction mixture was extracted with EtOAc $(3 \times 50 \mathrm{~mL})$, and the combined organic layers were washed with brine $(50 \mathrm{~mL})$, dried $\left(\mathrm{MgSO}_{4}\right)$, and concentrated. Flash column chromatography (silica gel, hexanes:EtOAc 20:1) yielded iodide 3 (1.8 g, 4.1 mmol, $92 \%$ yield) as a thick colorless oil. $3: \mathrm{R}_{f}=0.62$ (silica gel, hexanes:EtOAc 4:1); $[\alpha]_{\mathrm{D}}{ }^{32}=$ $-99.7\left(\mathrm{CDCl}_{3}, c=5.25\right)$; IR (film) $v_{\max } 2988,2954,2931,2857,1696,1589,1471,1462,1375$, $1348,1324,1250,1215,1196,1172,1120,1100,1055,1006,991,945,911,882,860,837,813$, 801, 779, $730 \mathrm{~cm}^{-1} ;{ }^{1} \mathrm{H}$ NMR (600 MHz, $\left.\mathrm{CDCl}_{3}\right): \delta=7.47$ (d, $\left.J=2.4 \mathrm{~Hz}, 1 \mathrm{H}\right), 4.52(\mathrm{~d}, J=2.4$ 
Hz, $1 \mathrm{H}), 4.21$ (s, $1 \mathrm{H}), 1.44$ (s, $3 \mathrm{H}), 1.42(\mathrm{~s}, 3 \mathrm{H}), 1.33$ (s, $3 \mathrm{H}), 0.91$ (s, $9 \mathrm{H}), 0.13$ (s, $3 \mathrm{H}), 0.11$ ppm (s, $3 \mathrm{H}) ;{ }^{13} \mathrm{C} \mathrm{NMR}\left(150 \mathrm{MHz}, \mathrm{CDCl}_{3}\right): \delta=186.9,161.9,110.9,99.2,85.2,79.4,74.5,27.7$, 27.0, 25.6, 19.2, 18.0, -4.6, -4.9 ppm; HRMS (ESI-TOF): calcd for $\mathrm{C}_{16} \mathrm{H}_{28} \mathrm{IO}_{4} \mathrm{Si}\left[\mathrm{M}+\mathrm{H}^{+}\right]$: 439.0796, found 439.0801 .

\section{Ullmann coupled product 12}

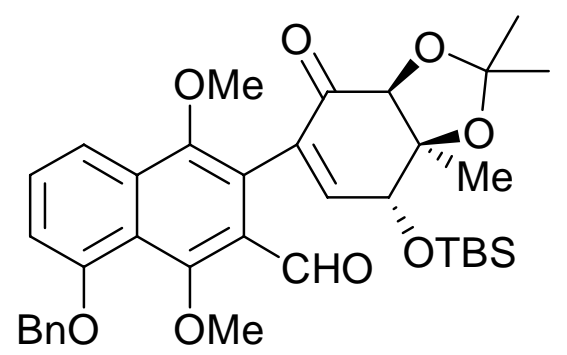

12

To a solution of bromo-aldehyde coupling partner $2(110 \mathrm{mg}$, $0.27 \mathrm{mmol}, 1.5$ equiv) and iodide coupling partner $3(80 \mathrm{mg}$, $0.18 \mathrm{mmol}, 1.0$ equiv) in DMSO $(2 \mathrm{~mL})$ at $25^{\circ} \mathrm{C}$ was added $\mathrm{Cu}$ powder (116 mg, $1.82 \mathrm{mmol}, 10.0$ equiv), $\mathrm{CuI}(14 \mathrm{mg}$, $0.073 \mathrm{mmol}, 0.4$ equiv), and $\mathrm{Pd}_{2}(\mathrm{dba})_{3}(17 \mathrm{mg}, 0.018 \mathrm{mmol}$, 0.1 equiv), and the reaction mixture was heated to $65^{\circ} \mathrm{C}$ for $2.5 \mathrm{~h}$ (iodide coupling partner 3 consumed by TLC), and then cooled to $25^{\circ} \mathrm{C}$. The reaction mixture was then quenched with $\mathrm{H}_{2} \mathrm{O}(5 \mathrm{~mL})$ and the biphasic reaction mixture was extracted with EtOAc $(3 \times 10 \mathrm{~mL})$, the combined organic layers were washed with brine $(10 \mathrm{~mL})$, dried $\left(\mathrm{MgSO}_{4}\right)$, and concentrated. Flash column chromatography (silica gel, hexanes:EtOAc 20:1) provided Ullmann coupled product $12(95 \mathrm{mg}, 0.15 \mathrm{mmol}, 83 \%$ yield) as a slightly yellow foam. 12: $\mathrm{R}_{f}=0.61$ (silica gel, hexanes:EtOAc $\left.2: 1\right) ;[\alpha]_{\mathrm{D}}^{32}=+8.1\left(\mathrm{CDCl}_{3}, c=1.25\right)$; IR (film) $v_{\max }$ 2987, 2951, 2932, 2881, 2857, 1696, 1673, 1609, 1570, 1495, 1445, 1403, 1363, 1329, 1250, 1212, 1196, 1124, 1083, 1054, 993, 973, 936, 888, 837, 819, 776, 734, $698 \mathrm{~cm}^{-1} ;{ }^{1} \mathrm{H}$ NMR $(600$ $\left.\mathrm{MHz}, \mathrm{CDCl}_{3}\right): \delta=10.4(\mathrm{~s}, 1 \mathrm{H}), 7.82(\mathrm{~d}, J=8.4 \mathrm{~Hz}, 1 \mathrm{H}), 7.59(\mathrm{t}, J=8.4 \mathrm{~Hz}, 1 \mathrm{H}), 7.56(\mathrm{~d}, J=$ $7.2 \mathrm{~Hz}, 2 \mathrm{H}), 7.44$ (t, $J=7.2 \mathrm{~Hz}, 2 \mathrm{H}), 7.38$ (t, J=7.2 Hz, $1 \mathrm{H}), 7.08$ (d, $J=8.4 \mathrm{~Hz}, 1 \mathrm{H}), 6.1$ (d, $J=1.8 \mathrm{~Hz}, 1 \mathrm{H}), 5.27$ (s, $2 \mathrm{H}), 4.74$ (s, $2 \mathrm{H}), 3.80(\mathrm{~s}, 3 \mathrm{H}), 3.71$ (s, $3 \mathrm{H}), 1.62$ (s, $3 \mathrm{H}), 1.58$ (s, 3 H), 1.52 (s, $3 \mathrm{H}), 0.95$ (s, $9 \mathrm{H}), 0.20$ (s, $3 \mathrm{H}), 0.17 \mathrm{ppm}(\mathrm{s}, 3 \mathrm{H}),{ }^{13} \mathrm{C} \mathrm{NMR}\left(150 \mathrm{MHz}, \mathrm{CDCl}_{3}\right): \delta$ $=192.5,191.1,191.1,161.5,156.6,151.7,148.4,136.3,135.1,133.6,130.3,128.7,128.2$, $127.6,123.7,122.5,120.5,116.0,109.9,109.5,84.6,82.1,73.6,71.6,65.8,61.8,27.9,26.5$, 
25.8, 19.6, 18.2, -4.5, -5.0 ppm; HRMS (ESI-TOF): calcd for $\mathrm{C}_{36} \mathrm{H}_{45} \mathrm{O}_{8} \mathrm{Si}\left[\mathrm{M}+\mathrm{H}^{+}\right]$: 633.2878 , found 633.2884 .

\section{Benzoin products 14 and 14a}

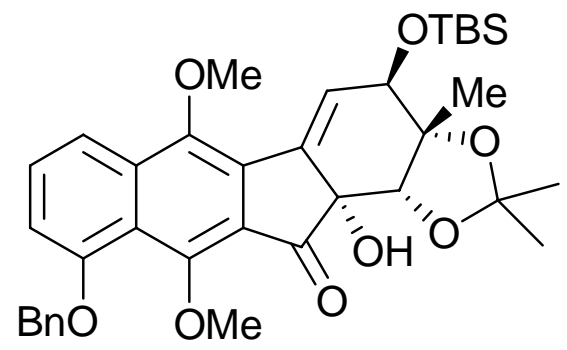

14

To a solution of Ullmann coupled product $12(60 \mathrm{mg}, 0.095$ mmol, 1.0 equiv) in $\mathrm{CH}_{2} \mathrm{Cl}_{2}(1 \mathrm{~mL})$ at $25^{\circ} \mathrm{C}$ was added Rovis catalyst 13 (6.9 mg, 0.019 mmol, 0.2 equiv), followed by $\mathrm{Et}_{3} \mathrm{~N}$ (26.4 $\mu \mathrm{L}, 0.19 \mathrm{mmol}, 2.0$ equiv). The reaction mixture was then heated to $45{ }^{\circ} \mathrm{C}$ for $4 \mathrm{~h}$ (consumption of starting material by TLC), and cooled to $25^{\circ} \mathrm{C}$. The reaction mixture was quenched with $\mathrm{H}_{2} \mathrm{O}(5 \mathrm{~mL})$, and the biphasic reaction mixture was extracted with EtOAc $(3 \times 5$ $\mathrm{mL})$, the combined organic layers were washed with brine $(5 \mathrm{~mL})$, dried $\left(\mathrm{MgSO}_{4}\right)$, and concentrated. Flash column chromatography (silica gel, hexanes:EtOAc 10:1) yielded benzoin products 14 and 14a (47 mg, $0.074 \mathrm{mmol}, 78 \%$ yield, 3:1 mixture of inconsequential isomers) as a slightly yellow oil. 14: $\mathrm{R}_{f}=0.63$ (silica gel, hexanes:EtOAc $\left.2: 1\right) ;[\alpha]_{\mathrm{D}}{ }^{32}=-51.8\left(\mathrm{CDCl}_{3}, c=\right.$ 1.48); IR (film) $v_{\max } 3504,2982,2951,2932,2881,2856,1719,1607,1569,1506,1445,1372$, $1355,1332,1277,1248,1212,1194,1112,1089,1059,1046,1029,1004,982,946,910,880$, $867,837,820,777,734,696 \mathrm{~cm}^{-1} ;{ }^{1} \mathrm{H}$ NMR (600 MHz, $\left.\mathrm{CDCl}_{3}\right): \delta=7.76(\mathrm{~d}, J=7.8 \mathrm{~Hz}, 1 \mathrm{H})$, $7.57(\mathrm{~d}, J=7.2 \mathrm{~Hz}, 1 \mathrm{H}), 7.56(\mathrm{~d}, J=7.2 \mathrm{~Hz}, 1 \mathrm{H}), 7.56$ (t, $J=7.8 \mathrm{~Hz}, 1 \mathrm{H}), 7.42(\mathrm{t}, J=7.2 \mathrm{~Hz}, 2$ H), $7.36(\mathrm{t}, J=7.2 \mathrm{~Hz}, 1 \mathrm{H}), 6.99(\mathrm{~d}, J=7.8 \mathrm{~Hz}, 1 \mathrm{H}), 6.76(\mathrm{~d}, J=3.0 \mathrm{~Hz}, 1 \mathrm{H}), 5.23(\mathrm{~s}, 2 \mathrm{H})$, $5.02(\mathrm{~d}, J=3.0 \mathrm{~Hz}, 1 \mathrm{H}), 4.10(\mathrm{~s}, 1 \mathrm{H}), 4.06(\mathrm{~s}, 1 \mathrm{H}), 3.94(\mathrm{~s}, 3 \mathrm{H}), 3.91(\mathrm{~s}, 3 \mathrm{H}), 1.60(\mathrm{~s}, 3 \mathrm{H})$, 1.52 (s, $3 \mathrm{H}), 1.32$ (s, $3 \mathrm{H}), 0.98$ (s, $9 \mathrm{H}), 0.18$ (s, $3 \mathrm{H}), 0.17 \mathrm{ppm}(\mathrm{s}, 3 \mathrm{H}) ;{ }^{13} \mathrm{C}$ NMR (150 MHz, $\left.\mathrm{CDCl}_{3}\right): \delta=199.9,158.5,55.9,147.6,136.6,136.5,136.3,131.4,130.3,130.1,128.6,128.0$ $127.5,122.3,122.0,115.0,112.9,108.7,88.2,81.1,75.7,73.7,71.3,63.0,60.0,60.1,28.1,27.8$, 25.9, 22.9, 18.3, -4.6, -5.1 ppm; HRMS (ESI-TOF): calcd for $\mathrm{C}_{36} \mathrm{H}_{45} \mathrm{O}_{8} \mathrm{Si}\left[\mathrm{M}+\mathrm{H}^{+}\right]$: 633.2878 , found 633.2899 . 


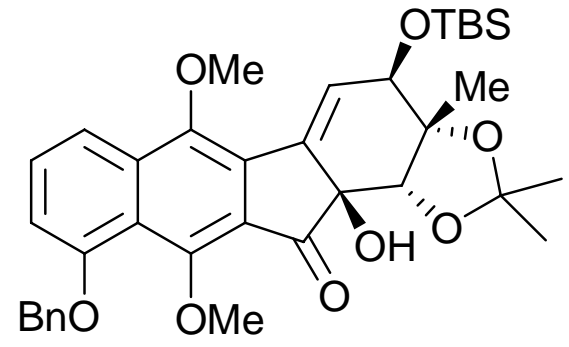

14a

14a: $\mathrm{R}_{f}=0.60$ (silica gel, hexanes:EtOAc $2: 1$ ); $[\alpha]_{\mathrm{D}}^{32}=+2.7$ $\left(\mathrm{CDCl}_{3}, c=0.98\right) ; \mathrm{IR}($ film $) v_{\max } 3391,2987,2932,2858$, $1725,1655,1607,1570,1507,1444,1373,1356,1333,1276$, 1257, 1213, 1179, 1148, 1115, 1048, 1016, 1003, 977, 962, 934, 910, 875, 831, 809, 780, 758, 732, $697 \mathrm{~cm}^{-1} ;{ }^{1} \mathrm{H}$ NMR $\left(600 \mathrm{MHz}, \mathrm{CDCl}_{3}\right): \delta=7.78(\mathrm{~d}, J=8.4 \mathrm{~Hz}, 1 \mathrm{H}), 7.58(\mathrm{~d}, J=$ $7.2 \mathrm{~Hz}, 2 \mathrm{H}), 7.56(\mathrm{t}, J=8.4 \mathrm{~Hz}, 1 \mathrm{H}), 7.42$ (t, $J=7.2 \mathrm{~Hz}, 2 \mathrm{H}), 7.35$ (t, $J=7.2 \mathrm{~Hz}, 1 \mathrm{H}), 7.15$ (d, $J=6.0 \mathrm{~Hz}, 1 \mathrm{H}), 7.00(\mathrm{~d}, J=8.4 \mathrm{~Hz}, 1 \mathrm{H}), 5.23(\mathrm{~s}, 2 \mathrm{H}), 4.79(\mathrm{~s}, 1 \mathrm{H}), 4.68(\mathrm{~s}, 1 \mathrm{H}), 4.31$ (d, $J=$ $6.0 \mathrm{~Hz}, 1 \mathrm{H}), 3.96(\mathrm{~s}, 3 \mathrm{H}), 3.93(\mathrm{~s}, 3 \mathrm{H}), 1.67(\mathrm{~s}, 3 \mathrm{H}), 1.32(\mathrm{~s}, 3 \mathrm{H}), 1.0(\mathrm{~s}, 3 \mathrm{H}), 0.90$ (s, $9 \mathrm{H})$, 0.22 (s, $3 \mathrm{H}), 0.21 \mathrm{ppm}(\mathrm{s}, 3 \mathrm{H}) ;{ }^{13} \mathrm{C} \mathrm{NMR}\left(150 \mathrm{MHz}, \mathrm{CDCl}_{3}\right): \delta=196.8,158.6,155.5,148.6$, $141.0,136.5,136.2,130.7,129.9,128.5,128.0,127.5,127.5,127.3,124.0,122.4,115.1,109.7$, $108.9,84.6,84.5,74.9,72.0,71.4,63.1,60.6,27.4,27.3,26.7,25.6,17.8,-4.1,-5.0$ ppm; HRMS (ESI-TOF): calcd for $\mathrm{C}_{36} \mathrm{H}_{45} \mathrm{O}_{8} \mathrm{Si}\left[\mathrm{M}+\mathrm{H}^{+}\right]$: 633.2878, found 633.2874.

\section{Indenone 14b}

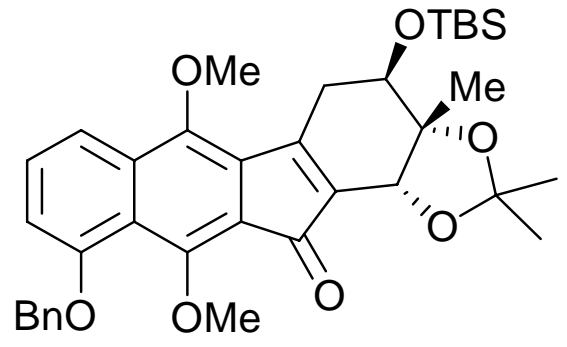

14b
To a solution of benzoin products 14 and 14a (200 mg, 0.31 mmol, 1.0 equiv) in $\mathrm{CH}_{2} \mathrm{Cl}_{2}(4 \mathrm{~mL})$ at $25{ }^{\circ} \mathrm{C}$ was added $\mathrm{Ac}_{2} \mathrm{O}$ (322 mg, $3.1 \mathrm{mmol}, 10.0$ equiv), DMAP (38 mg, $0.31 \mathrm{mmol}$, 1.0 equiv) and $\mathrm{Et}_{3} \mathrm{~N}$ (319 mg, $3.1 \mathrm{mmol}, 10.0$ equiv). The reaction mixture was stirred at $25^{\circ} \mathrm{C}$ for $20 \mathrm{~h}$, and then diluted with ethyl acetate $(50 \mathrm{~mL})$. The biphasic reaction mixture was extracted with EtOAc $(3 \times 50 \mathrm{~mL})$, and the combined organic layers were washed with sat. aqueous $\mathrm{NaHCO}_{3}(100 \mathrm{~mL})$ and brine $(100 \mathrm{~mL})$, then dried $\left(\mathrm{Na}_{2} \mathrm{SO}_{4}\right)$, and concentrated. Flash chromatography (silica gel, hexanes:EtOAc 16:1) provided the corresponding acetate protected benzoin product (202 mg, $0.30 \mathrm{mmol}, 95 \%$ yield) as a slightly yellow foam. To a solution of the acetate protected benzoin product $\left(190 \mathrm{mg}, 0.28 \mathrm{mmol}, 1.0\right.$ equiv) in THF $(5 \mathrm{~mL})$ at $-78{ }^{\circ} \mathrm{C}$ was added $\mathrm{MeOH}\left(57 \mu \mathrm{L}, 1.4 \mathrm{mmol}, 5.0\right.$ equiv), followed by $\mathrm{SmI}_{2}(5.6 \mathrm{~mL}, 0.1 \mathrm{M}$ in THF, 0.56 
mmol, 2.0 equiv) added dropwise. The reaction mixture was stirred at $-78{ }^{\circ} \mathrm{C}$ for $10 \mathrm{~min}$ (starting material consumed by TLC), then quenched with sat. aqueous $\mathrm{NH}_{4} \mathrm{Cl}(10 \mathrm{~mL})$, and the biphasic reaction mixture was extracted with EtOAc $(3 \times 10 \mathrm{~mL})$. The combined organic layers

were washed with sat. aqueous $\mathrm{NH}_{4} \mathrm{Cl}(10 \mathrm{~mL})$ and brine $(10 \mathrm{~mL})$, then dried $\left(\mathrm{Na}_{2} \mathrm{SO}_{4}\right)$, and concentrated. The residual oil was dissolved in $\mathrm{CH}_{2} \mathrm{Cl}_{2}(8 \mathrm{~mL})$, and $\mathrm{Et}_{3} \mathrm{~N}(77 \mu \mathrm{L}, 0.56 \mathrm{mmol}, 2.0$ equiv) was added at $25{ }^{\circ} \mathrm{C}$. The reaction mixture was stirred at $25{ }^{\circ} \mathrm{C}$ for $1 \mathrm{~h}$ and then concentrated. Flash chromatography (silica gel, hexanes:EtOAc 18:1) afforded indenone 14b (141 mg, $0.23 \mathrm{mmol}, 81 \%$ yield over two steps) as a slightly yellow foam. 14b: $\mathrm{R}_{f}=0.60$ (silica gel, hexanes:EtOAc 4:1); $[\alpha]_{\mathrm{D}}{ }^{32}=+16.1\left(\mathrm{CDCl}_{3}, c=1.02\right)$; IR (film) $v_{\max } 2977,2932,2891$, 2856, 1694, 1591, 1525, 1446, 1372, 1346, 1327, 1248, 1214, 1194, 1121, 1060, 1044, 1018, 980, 908, 864, 878, 837, 816, 778, 761, 733, $697 \mathrm{~cm}^{-1} ;{ }^{1} \mathrm{H}$ NMR $\left(600 \mathrm{MHz}, \mathrm{CDCl}_{3}\right): \delta=7.61(\mathrm{~d}$, $J=8.4 \mathrm{~Hz}, 1 \mathrm{H}), 7.55(\mathrm{~d}, J=7.8 \mathrm{~Hz}, 2 \mathrm{H}), 7.49(\mathrm{t}, J=8.4 \mathrm{~Hz}, 1 \mathrm{H}), 7.42(\mathrm{t}, J=7.8 \mathrm{~Hz}, 2 \mathrm{H})$, 7.35 (t, $J=7.8 \mathrm{~Hz}, 1 \mathrm{H}), 7.03$ (d, J=8.4 Hz, $1 \mathrm{H}), 5.20$ (q, $J=11.4 \mathrm{~Hz}, 2 \mathrm{H}), 4.68$ (s, $1 \mathrm{H}), 4.14$ (br dd, $J=9.6,5.4 \mathrm{~Hz}, 1 \mathrm{H}), 3.92$ (s, $3 \mathrm{H}), 3.91$ (s, $3 \mathrm{H}), 3.26(\mathrm{dd}, J=19.2,5.4 \mathrm{~Hz}, 1 \mathrm{H}), 2.64$ (dd, $J=19.2,9.6 \mathrm{~Hz}, 1 \mathrm{H}), 1.52(\mathrm{~s}, 3 \mathrm{H}), 1.49(\mathrm{~s}, 3 \mathrm{H}), 1.30(\mathrm{~s}, 3 \mathrm{H}), 0.95$ (s, $9 \mathrm{H}), 0.16(\mathrm{~s}, 3 \mathrm{H})$, 0.14 ppm (s, $3 \mathrm{H}) ;{ }^{13} \mathrm{C}$ NMR (150 MHz, $\left.\mathrm{CDCl}_{3}\right): \delta=190.9,159.1,156.7,154.0,147.1,136.6$, $135.8,133.1,129.9,128.5,128.0,127.5,127.4,123.0,119.3,116.0,111.1,109.7,82.1,73.9$, 72.3, 71.5, 63.2, 62.8, 33.3, 28.7, 27.2, 25.8, 18.1, 17.2, -4.4, -4.6 ppm; HRMS (ESI-TOF): calcd for $\mathrm{C}_{36} \mathrm{H}_{45} \mathrm{O}_{7} \mathrm{Si}\left[\mathrm{M}+\mathrm{H}^{+}\right]$: 617.2929, found 617.2929.

\section{Allylic alcohol 15}

To a suspension of indenone $\mathbf{1 4 b}(696 \mathrm{mg}, 1.13 \mathrm{mmol}, 1.0 \mathrm{eq})$ in 1,4-dioxane $(40 \mathrm{~mL})$ at $25{ }^{\circ} \mathrm{C}$ was added $\mathrm{SeO}_{2}\left(145 \mathrm{mg}, 1.30 \mathrm{mmol}, 1.2\right.$ equiv), and the reaction mixture was heated at $110{ }^{\circ} \mathrm{C}$ for $9 \mathrm{~h}$. Then reaction mixture was then cooled to $25^{\circ} \mathrm{C}$, and quenched with sat. aqueous $\mathrm{Na}_{2} \mathrm{SO}_{3}(50 \mathrm{~mL})$ and the biphasic reaction mixture was extracted with EtOAc $(3 \times 50 \mathrm{~mL})$. The combined organic layers were washed with brine $(100 \mathrm{~mL})$, dried $\left(\mathrm{MgSO}_{4}\right)$, and concentrated. Flash chromatography chromatography (silica gel, hexanes:EtOAc 18:1) gave allylic alcohol 15 


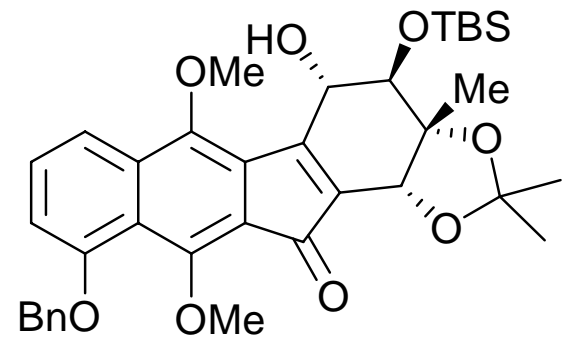

15

(515 mg, $0.81 \mathrm{mmol}, 72 \%$ yield) as slightly yellow foam. 15: $\mathrm{R}_{f}=0.60$ (silica gel, hexanes:EtOAc 2:1); $[\alpha]_{\mathrm{D}}{ }^{32}=+7.9$ $\left(\mathrm{CDCl}_{3}, c=0.67\right)$; IR (film) $v_{\max } 3453,2932,2855,1697$, $1590,1448,1370,1346,1320,1251,1214,1124,1081,1055$, 1036, 1004, 878, 838, 779, 737, $698 \mathrm{~cm}^{-1} ;{ }^{1} \mathrm{H}$ NMR $(600$ $\left.\mathrm{MHz}, \mathrm{CDCl}_{3}\right): \delta=7.59(\mathrm{~d}, J=8.4 \mathrm{~Hz}, 1 \mathrm{H}), 7.55(\mathrm{~d}, J=7.2$ Hz, $2 \mathrm{H}), 7.52(\mathrm{t}, J=8.4 \mathrm{~Hz}, 1 \mathrm{H}), 7.42(\mathrm{t}, J=7.2 \mathrm{~Hz}, 2 \mathrm{H}), 7.36(\mathrm{t}, J=7.2 \mathrm{~Hz}, 1 \mathrm{H}), 7.05(\mathrm{~d}, J=$ $8.4 \mathrm{~Hz}, 1 \mathrm{H}), 5.21(\mathrm{q}, J=11.4 \mathrm{~Hz}, 2 \mathrm{H}), 4.66(\mathrm{~s}, 1 \mathrm{H}), 4.64$ (d, J = 3.6 Hz, $1 \mathrm{H}), 4.48-4.95$ (brs, 1 H), $4.08(\mathrm{~d}, J=6.6 \mathrm{~Hz}, 1 \mathrm{H}), 4.02(\mathrm{~s}, 3 \mathrm{H}), 3.93(\mathrm{~s}, 3 \mathrm{H}), 1.51(\mathrm{~s}, 3 \mathrm{H}), 1.49$ (s, $3 \mathrm{H}), 1.35$ (s, 3 $\mathrm{H}), 0.95(\mathrm{~s}, 9 \mathrm{H}), 0.20(\mathrm{~s}, 3 \mathrm{H}), 0.18 \mathrm{ppm}(\mathrm{s}, 3 \mathrm{H}) ;{ }^{13} \mathrm{C} \mathrm{NMR}\left(150 \mathrm{MHz}, \mathrm{CDCl}_{3}\right): \delta=190.9$, $159.2,155.8,155.0,145.2,136.4,135.0,132.3,128.7,128.66,128.0,127.5,127.1,122.9,119.0$, $116.0,111.2,110.8,83.0,73.5,71.5,69.7,63.8,62.9,28.6,27.7,25.9,18.9,18.4,-4.2,-4.9$ ppm; HRMS (ESI-TOF): calcd for $\mathrm{C}_{36} \mathrm{H}_{45} \mathrm{O}_{8} \mathrm{Si}\left[\mathrm{M}+\mathrm{H}^{+}\right]$: 633.2878, found 633.2872.

\section{Acetate protected compound 15a}

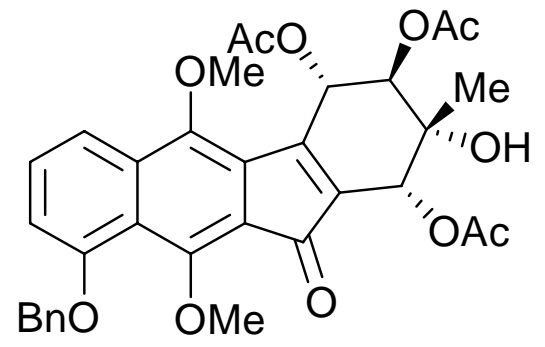

$15 \mathbf{a}$

To a solution of allylic alcohol $15(70 \mathrm{mg}, 0.11 \mathrm{mmol}, 1.0$ equiv) in $\mathrm{MeCN}(7 \mathrm{~mL})$ at $25^{\circ} \mathrm{C}$ was added aq. $\mathrm{HF}(2.1 \mathrm{~mL})$ and the reaction mixture was stirred at $25{ }^{\circ} \mathrm{C}$ for $3 \mathrm{~h}$. The reaction mixture was then quenched with brine $(20 \mathrm{~mL})$ and extracted with EtOAc $(3 \times 25 \mathrm{~mL})$. The combined organic layers were washed with sat. aq. $\mathrm{NaHCO}_{3}(2 \times 25 \mathrm{~mL})$ and brine $(25 \mathrm{~mL})$, dried $\left(\mathrm{MgSO}_{4}\right)$, and concentrated. The resulting oil was dissolved in $\mathrm{CH}_{2} \mathrm{Cl}_{2}$ (3 $\mathrm{mL})$, and $\mathrm{Ac}_{2} \mathrm{O}\left(105 \mu \mathrm{L}, 1.11 \mathrm{mmol}, 10.0\right.$ equiv) was added at $25{ }^{\circ} \mathrm{C}$, followed by DMAP (14 $\mathrm{mg}, 0.11 \mathrm{mmol}, 1.0$ equiv), and $\mathrm{Et}_{3} \mathrm{~N}(155 \mu \mathrm{L}, 1.11 \mathrm{mmol}, 10.0$ equiv). The reaction mixture was stirred for $30 \mathrm{~min}$ at $25^{\circ} \mathrm{C}$, and then quenched with brine $(10 \mathrm{~mL})$ and extracted with EtOAc $(3 \times 10 \mathrm{~mL})$. The combined organic layers were washed with brine $(10 \mathrm{~mL})$, dried $\left(\mathrm{MgSO}_{4}\right)$, and concentrated. Flash column chromatography (silica gel, hexanes:EtOAc 2:1) provided acetate 
protected 15a (60 mg, $0.099 \mathrm{mmol}, 89 \%$ yield over two steps) as a yellow-orange foam. 15a: $\mathrm{R}_{f}$ $=0.20$ (silica gel, hexanes:EtOAc 1:1); $[\alpha]_{\mathrm{D}}^{32}=-65.0\left(\mathrm{CDCl}_{3}, c=0.93\right) ;$ IR (film) $v_{\max } 3476$, 2931, 2850, 1747, 1695, 1590, 1525, 1446, 1371, 1345, 1321, 1224, 1046, 1026, 976, 911, 844, 810, 758, 732, $699 \mathrm{~cm}^{-1} ;{ }^{1} \mathrm{H}$ NMR $\left(500 \mathrm{MHz}, \mathrm{CDCl}_{3}\right): \delta=7.47(\mathrm{~d}, J=8.0 \mathrm{~Hz}, 1 \mathrm{H}), 7.54(\mathrm{~d}, J=$ $7.5 \mathrm{~Hz}, 2 \mathrm{H}), 7.50$ (t, $J=8.0 \mathrm{~Hz}, 1 \mathrm{H}), 7.40$ (t, $J=7.5 \mathrm{~Hz}, 2 \mathrm{H}), 7.34$ (t, $J=7.5 \mathrm{~Hz}, 1 \mathrm{H}), 7.04$ (d, $J=8.0 \mathrm{~Hz}, 1 \mathrm{H}), 6.05(\mathrm{~d}, J=6.0 \mathrm{~Hz}, 1 \mathrm{H}), 5.90(\mathrm{~s}, 1 \mathrm{H}), 5.49$ (d, $J=6.0 \mathrm{~Hz}, 1 \mathrm{H}), 5.20(\mathrm{~s}, 2 \mathrm{H})$, 3.88 (s, $3 \mathrm{H}), 3.79$ (s, $3 \mathrm{H}), 2.17(\mathrm{~s}, 3 \mathrm{H}), 2.16$ (s, $3 \mathrm{H}), 2.13(\mathrm{~s}, 3 \mathrm{H}), 1.36 \mathrm{ppm}(\mathrm{s}, 3 \mathrm{H}) ;{ }^{13} \mathrm{C}$ NMR (125 MHz, $\left.\mathrm{CDCl}_{3}\right): \delta=189.0,170.7,170.3,170.2,159.1,155.0,147.3,136.4,135.6$, $130.3,128.5,128.0,127.4,125.2,122.9,118.9,116.6,111.5,75.1,72.4,71.5,67.9,67.5,63.9$, 62.7, 31.5, 22.6, 20.9, 20.2, $14.1 \mathrm{ppm}$; HRMS (ESI-TOF): calcd for $\mathrm{C}_{33} \mathrm{H}_{33} \mathrm{O}_{11}\left[\mathrm{M}+\mathrm{H}^{+}\right]$: 605.2017, found 605.1999.

\section{Free-phenol 16}

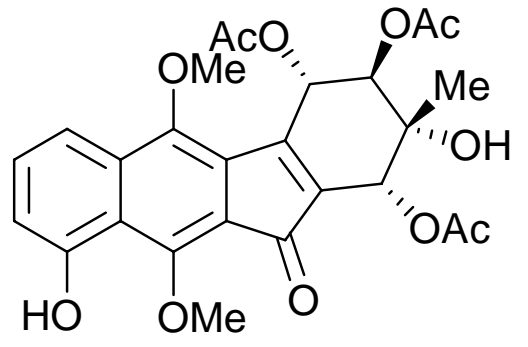

16

To a solution of acetate protected 15a $(57 \mathrm{mg}, 0.094 \mathrm{mmol}, 1.0$ equiv) in EtOAc $(3 \mathrm{~mL})$ and $\mathrm{AcOH}(10 \mu \mathrm{L})$ at $25^{\circ} \mathrm{C}$ was added $\mathrm{Pd} / \mathrm{C}(5.7 \mathrm{mg}, 10 \% \mathrm{w} / \mathrm{w})$, and the reaction mixture was stirred at $25{ }^{\circ} \mathrm{C}$ under a hydrogen atmosphere (balloon, $1 \mathrm{~atm}$ ) for $3 \mathrm{~h}$ (checking each hour by ${ }^{1} \mathrm{H}$ NMR). Upon consumption of starting material ( ${ }^{1} \mathrm{H}$ NMR), the reaction mixture was filtered through a plug of Celite, and concentrated. The resulting free-phenol 16 (48 mg, $0.093 \mathrm{mmol}, 99 \%$ yield) was used without further purification. 16: $\mathrm{R}_{f}=0.24$ (silica gel, hexanes:EtOAc 1:1); $[\alpha]_{\mathrm{D}}{ }^{32}=-96.6\left(\mathrm{CDCl}_{3}, c=\right.$ 0.5); IR (film) $v_{\max } 3473,3362,2941,1748,1693,1590,1532,1444,1371,1346,1225,1165$, 1036, 1019, 930, 907, 816, $730 \mathrm{~cm}^{-1} ;{ }^{1} \mathrm{H}$ NMR $\left(600 \mathrm{MHz}, \mathrm{CDCl}_{3}\right): \delta=9.74(\mathrm{~s}, 1 \mathrm{H}), 7.49(\mathrm{t}, J=$ $7.8 \mathrm{~Hz}, 1 \mathrm{H}), 7.44$ (d, $J=7.8 \mathrm{~Hz}, 1 \mathrm{H}), 6.97$ (d, $J=7.8 \mathrm{~Hz}, 1 \mathrm{H}), 6.04$ (d, $J=6.6 \mathrm{~Hz}, 1 \mathrm{H}), 5.89$ (s, $1 \mathrm{H}), 5.49$ (d, J = 6.6 Hz, $1 \mathrm{H}), 4.34$ (s, $3 \mathrm{H}), 3.80$ (s, $3 \mathrm{H}), 2.19$ (s, $3 \mathrm{H}), 2.17$ (s, $3 \mathrm{H}), 2.12$ (s, $3 \mathrm{H}), 1.35 \mathrm{ppm}(\mathrm{s}, 3 \mathrm{H}) ;{ }^{13} \mathrm{C} \mathrm{NMR}\left(125 \mathrm{MHz}, \mathrm{CDCl}_{3}\right): \delta=189.0,170.7,170.3,170.2,159.1$, 155.0, 147.3, 136.4, 135.6, 188.1, 170.3, 170.2, 158.7, 153.9, 147.9, 135.4, 134.2, 131.7, 124.7, 
118.1, 115.4, 115.2, 75.1, 72.4, 67.8, 67.4, 64.4, 63.9, 21.0, 20.9, 20.2 ppm; HRMS (ESI-TOF): calcd for $\mathrm{C}_{26} \mathrm{H}_{26} \mathrm{O}_{11} \mathrm{Na}\left[\mathrm{M}+\mathrm{Na}^{+}\right]$: 537.1367, found 537.1375.

\section{TBS-protected phenol 16a}

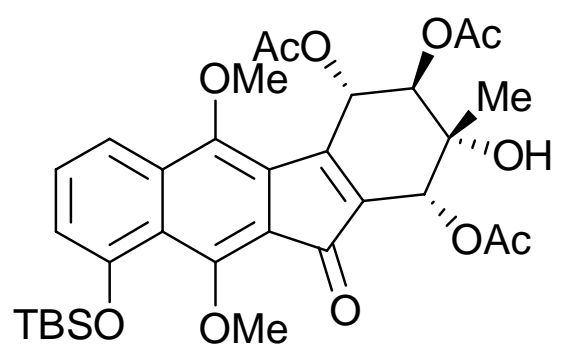

16a

To a solution of free-phenol $16(257 \mathrm{mg}, 0.50 \mathrm{mmol}, 1.0$ equiv) in DMF $(9 \mathrm{~mL})$ at $25^{\circ} \mathrm{C}$ was added $\mathrm{TBSCl}(375 \mathrm{mg}$, $2.5 \mathrm{mmol}, 5.0$ equiv) and imid. (204 $\mathrm{mg}, 3.0 \mathrm{mmol}, 6.0$ equiv) and the reaction mixture was stirred at $25^{\circ} \mathrm{C}$ for $3 \mathrm{~h}$. The reaction mixture was quenched with $\mathrm{H}_{2} \mathrm{O}(20 \mathrm{~mL})$ and the biphasic reaction mixture was extracted with EtOAc $(3 \times 20$ $\mathrm{mL})$, and the combined organic layers were washed with sat. aqueous $\mathrm{NaHCO}_{3}(20 \mathrm{~mL})$ and brine $(20 \mathrm{~mL})$, then dried $\left(\mathrm{Na}_{2} \mathrm{SO}_{4}\right)$, and concentrated. Flash chromatography (silica gel, hexanes:EtOAc 2:1) afforded TBS-protected phenol 16a (296 mg, $0.47 \mathrm{mmol}, 94 \%$ yield) as yellow foam. 16a: $\mathrm{R}_{f}=0.30$ (silica gel, hexanes:EtOAc $\left.1: 1\right) ;[\alpha]_{\mathrm{D}}{ }^{32}=-108.3\left(\mathrm{CDCl}_{3}, c=0.65\right)$; IR (film) $v_{\max } 3484,2932,2857,1747,1695,1590,1524,1447,1370,1344,1220,1038,1022$, 977, 956, 938, 910, 840, 821, 804, 760, $730 \mathrm{~cm}^{-1} ;{ }^{1} \mathrm{H}$ NMR $\left(600 \mathrm{MHz}, \mathrm{CDCl}_{3}\right): \delta=7.56(\mathrm{~d}, J=$ $7.8 \mathrm{~Hz}, 1 \mathrm{H}), 7.43$ (t, $J=7.8 \mathrm{~Hz}, 1 \mathrm{H}), 6.93$ (d, $J=7.8 \mathrm{~Hz}, 1 \mathrm{H}), 6.04$ (d, $J=6.0 \mathrm{~Hz}, 1 \mathrm{H}), 5.89$ (s, $1 \mathrm{H}), 5.48(\mathrm{~d}, J=6.0 \mathrm{~Hz}, 1 \mathrm{H}), 4.00(\mathrm{~s}, 3 \mathrm{H}), 3.79(\mathrm{~s}, 3 \mathrm{H}), 2.17(\mathrm{~s}, 3 \mathrm{H}), 2.16(\mathrm{~s}, 3 \mathrm{H}), 2.12$ (s, $3 \mathrm{H}), 1.35$ (s, $3 \mathrm{H}), 1.02(\mathrm{~s}, 9 \mathrm{H}), 0.25$ (s, $3 \mathrm{H}), 0.23$ ppm (s, $3 \mathrm{H}) ;{ }^{13} \mathrm{C} \mathrm{NMR}\left(125 \mathrm{MHz}, \mathrm{CDCl}_{3}\right)$ : $\delta=188.9,170.8,170.3,170.3,156.1,155.6,147.2,135.5,135.4,130.1,124.8,124.6,119.9$, 117.6, 117.0, 75.2, 72.4, 67.9, 67.5, 63.9, 62.9, 26.1, 21.0, 21.0, 21.0, 20.2, 18.8, -4.1, -4.2 ppm; HRMS (ESI-TOF): calcd for $\mathrm{C}_{32} \mathrm{H}_{41} \mathrm{O}_{11} \mathrm{Si}\left[\mathrm{M}+\mathrm{H}^{+}\right]$: 629.2413, found 629.2412.

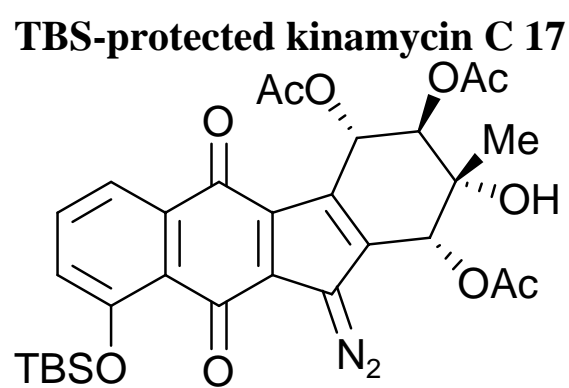

17

To a solution of TBS-protected phenol 16a $(25 \mathrm{mg}, 0.040$ mmol, 1.0 equiv) in $i-\operatorname{PrOH}(2.0 \mathrm{~mL})$ at $25{ }^{\circ} \mathrm{C}$ was added $\mathrm{TsHNNH}_{2}$ (37 mg, $0.20 \mathrm{mmol}, 5.0$ equiv), and aq. $1 \mathrm{M} \mathrm{HCl}$ 
$(0.05 \mathrm{~mL})$, and the reaction mixture was stirred at that temperature for $18 \mathrm{~h}$. The reaction mixture was quenched with $\mathrm{H}_{2} \mathrm{O}(2 \mathrm{~mL})$ and the biphasic reaction mixture was extracted with EtOAc $(3 \times 2 \mathrm{~mL})$, and the combined organic layers were dried $\left(\mathrm{MgSO}_{4}\right)$, filtered, and concentrated. Preparative plate chromatography (silica gel, benzene:acetone 5:1) provided the corresponding tosyl-hydrazone (30 $\mathrm{mg}, 0.038 \mathrm{mmol}, 95 \%$ yield, ca. 4:1 mixture of isomers) as a yellow-orange foam. To a cold $\left(0^{\circ} \mathrm{C}\right)$ solution of the tosyl-hydrazone $(27 \mathrm{mg}, 0.034 \mathrm{mmol}, 1.0$ equiv) in MeCN (1.0 mL) was added CAN (56 mg, $0.102 \mathrm{mmol}, 3.0$ equiv) in pH 7 buffer (0.1 $\mathrm{mL}$ ). The reaction mixture was stirred at $0{ }^{\circ} \mathrm{C}$ for about $1 \mathrm{~h}$ (consumption of starting material by TLC), and then put directly on a prep-plate (silica gel, hexanes:EtOAc 1:1), which yielded TBSprotected kinamycin $\mathrm{C}(\mathbf{1 7}, 8.7 \mathrm{mg}, 0.014 \mathrm{mmol}, 42 \%$ yield $)$ as a yellow foam. $17: \mathrm{R}_{f}=0.30$ (silica gel, hexanes:EtOAc 1:1); $[\alpha]_{\mathrm{D}}{ }^{32}=-37.8\left(\mathrm{CDCl}_{3}, c=0.33\right)$; IR (film) $v_{\max } 3463,2951$, 2931, 2860, 2144, 1744, 1651, 1582, 1486, 1462, 1433, 1371, 1319, 1297, 1226, 1165, 1085, 1046, 968, 881, 843, 821, 806, 789, $732 \mathrm{~cm}^{-1} ;{ }^{1} \mathrm{H}$ NMR $\left(600 \mathrm{MHz}, \mathrm{CDCl}_{3}\right): \delta=7.83(\mathrm{~d}, J=7.8$ $\mathrm{Hz}, 1 \mathrm{H}), 7.50(\mathrm{t}, J=7.8 \mathrm{~Hz}, 1 \mathrm{H}), 7.12(\mathrm{~d}, J=7.8 \mathrm{~Hz}, 1 \mathrm{H}), 6.23(\mathrm{~d}, J=7.2 \mathrm{~Hz}, 1 \mathrm{H}), 5.60(\mathrm{~d}, J$ $=7.2 \mathrm{~Hz}, 1 \mathrm{H}), 5.50(\mathrm{~s}, 1 \mathrm{H}), 2.19(\mathrm{~s}, 6 \mathrm{H}), 2.12(\mathrm{~s}, 3 \mathrm{H}), 1.28(\mathrm{~s}, 3 \mathrm{H}), 1.05(\mathrm{~s}, 9 \mathrm{H}), 0.26 \mathrm{ppm}(\mathrm{s}$, $6 \mathrm{H}) ;{ }^{13} \mathrm{C} \mathrm{NMR}\left(125 \mathrm{MHz}, \mathrm{CDCl}_{3}\right): \delta=178.6,178.5,172.0,170.9,170.3,156.4,136.5,136.0$, $134.1,128.4,127.7,127.3,125.4,122.8,121.2,78.0,75.6,73.6,71.1,68.2,25.9,21.1,21.0$, 20.8, 18.6, 18.6, -4.1, -4.1 ppm; HRMS (ESI-TOF): calcd for $\mathrm{C}_{30} \mathrm{H}_{35} \mathrm{~N}_{2} \mathrm{O}_{10} \mathrm{Si}\left[\mathrm{M}+\mathrm{H}^{+}\right]$: 611.2056, found 611.2039.

\section{Kinamycin C (1c)}

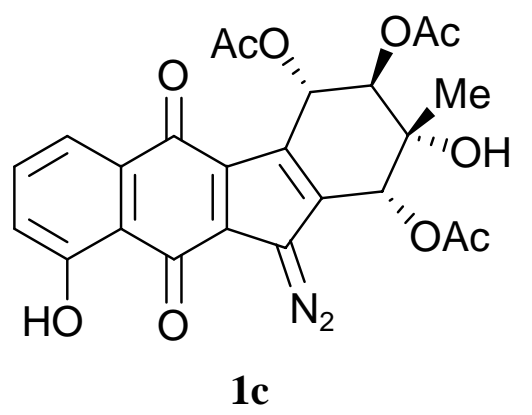

To a solution of TBS-protected kinamycin C (17, $8.0 \mathrm{mg}, 0.013$ mmol, 1.0 equiv) in $\mathrm{MeCN}(0.2 \mathrm{~mL})$ at $25{ }^{\circ} \mathrm{C}$ was added aq. $1 \mathrm{M}$ $\mathrm{HCl}(0.1 \mathrm{~mL})$, and the reaction mixture was stirred at $25^{\circ} \mathrm{C}$ for 3 h. The reaction mixture was then quenched with $\mathrm{H}_{2} \mathrm{O}(0.5 \mathrm{~mL})$, the biphasic reaction mixture was extracted with EtOAc $(3 \times 0.5$ $\mathrm{mL})$, and the combined organic layers were dried $\left(\mathrm{MgSO}_{4}\right)$, and 
concentrated. Preparative-plate chromatography (silica gel, hexanes:EtOAc 1:1) gave kinamycin C (1c, $6.1 \mathrm{mg}, 0.012 \mathrm{mmol}, 95 \%$ yield) as a yellow foam. 1c: $\mathrm{R}_{f}=0.23$ (silica gel, hexanes:EtOAc 1:1); $[\alpha]_{\mathrm{D}}^{32}=-23.8\left(\mathrm{CHCl}_{3}, c=0.34\right)$; IR (film) $v_{\max } 3470,2927,2850,2148$, $1741,1659,1623,1597,1484,1457,1370,1331,1301,1229,1157,1075,1039,1019,953,908$, 833, 765, 753, 734, $701 \mathrm{~cm}^{-1}$; ${ }^{1} \mathrm{H}$ NMR $\left(600 \mathrm{MHz}, \mathrm{CDCl}_{3}\right): \delta=12.12(\mathrm{~s}, 1 \mathrm{H}), 7.67(\mathrm{~d}, J=8.4$ Hz, $1 \mathrm{H}), 7.56$ (t, $J=8.4 \mathrm{~Hz}, 1 \mathrm{H}), 7.18(\mathrm{~d}, J=8.4 \mathrm{~Hz}, 1 \mathrm{H}), 6.23$ (d, $J=7.2 \mathrm{~Hz}, 1 \mathrm{H}), 5.60$ (d, $J$ $=7.2 \mathrm{~Hz}, 1 \mathrm{H}), 5.49(\mathrm{~s}, 1 \mathrm{H}), 2.66(\mathrm{br} \mathrm{s}, 1 \mathrm{H}), 2.21(\mathrm{~s}, 3 \mathrm{H}), 2.20(\mathrm{~s}, 3 \mathrm{H}), 2.11(\mathrm{~s}, 3 \mathrm{H}), 1.27 \mathrm{ppm}$ (s, $3 \mathrm{H}) ;{ }^{13} \mathrm{C}$ NMR $\left(125 \mathrm{MHz}, \mathrm{CDCl}_{3}\right): \delta=184.0,178.1,171.9,171.0,162.0,136.3,134.3$, 132.6, 130.0, 129.1, 126.7, 123.8, 119.9, 115.6, 77.9, 75.5, 73.6, 71.0, 68.0, 21.1, 21.0, 20.8, 18.6 ppm; HRMS (ESI-TOF): calcd for $\mathrm{C}_{24} \mathrm{H}_{21} \mathrm{~N}_{2} \mathrm{O}_{10}\left[\mathrm{M}+\mathrm{H}^{+}\right]$: 497.1196, found 497.1195.

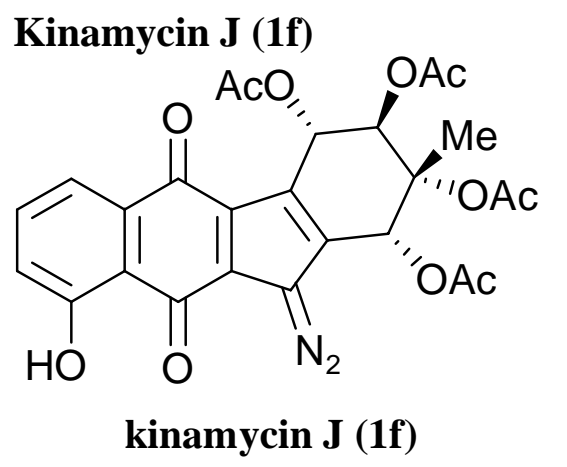

To a solution of TBS-protected kinamycin C $17(3.2 \mathrm{mg}$, 0.0052 mmol, 1.0 equiv) in $\mathrm{CH}_{2} \mathrm{Cl}_{2}(0.2 \mathrm{~mL})$ at $25{ }^{\circ} \mathrm{C}$ was added $\mathrm{Ac}_{2} \mathrm{O}(5.3 \mu \mathrm{L}, 0.052 \mathrm{mmol}, 10.0$ equiv), DMAP (0.64 $\mathrm{mg}, 0.0052 \mathrm{mmol}, 1.0$ equiv), and $\mathrm{Et}_{3} \mathrm{~N}(7.2 \mu \mathrm{L}, 0.052 \mathrm{mmol}$, 10.0 equiv). The reaction mixture was stirred at $25^{\circ} \mathrm{C}$ for $2 \mathrm{~h}$, and then put directly onto a prep-plate (silica gel, hexanes:EtOAc 1:1), which afforded the corresponding tetraacetate as a yellow foam. This foam was then dissolved in $\mathrm{MeCN}(0.2 \mathrm{~mL})$, and aq. $1 \mathrm{M} \mathrm{HCl}(0.1 \mathrm{~mL})$ was added to the reaction mixture at $25^{\circ} \mathrm{C}$. The reaction mixture was then stirred for $3 \mathrm{~h}$ at $25{ }^{\circ} \mathrm{C}$ and then quenched with $\mathrm{H}_{2} \mathrm{O}(0.5 \mathrm{~mL})$, and the biphasic reaction mixture was extracted with EtOAc $(3 \times 0.5 \mathrm{~mL})$, and the combined organic layers were dried $\left(\mathrm{MgSO}_{4}\right)$, and concentrated. Preparative plate chromatography (silica gel, $\mathrm{CH}_{2} \mathrm{Cl}_{2}: \mathrm{MeOH}$ 9:1) provided kinamycin $\mathrm{J}$ (1f, $2.2 \mathrm{mg}, 0.0041 \mathrm{mmol}, 80 \%$ yield) as a yellow foam. 1f: $\mathrm{R}_{f}=0.52$ (silica gel, hexanes:EtOAc 1:1); $[\alpha]_{\mathrm{D}}^{32}=-75.0\left(\mathrm{CDCl}_{3}, c=0.06\right)$; IR (film) $v_{\max } 2950,2924$, 2852, 2156, 1743, 1660, 1624, 1456, 1370, 1258, 1229, 1166, 1077, 1036, 1021, 909, 801, 762, 733, $701 \mathrm{~cm}^{-1} ;{ }^{1} \mathrm{H}$ NMR $\left(600 \mathrm{MHz}, \mathrm{CDCl}_{3}\right): \delta=12.14(\mathrm{~s}, 1 \mathrm{H}), 7.67(\mathrm{~d}, J=7.8 \mathrm{~Hz}, 1 \mathrm{H}), 7.55(\mathrm{t}$, 
$J=7.8 \mathrm{~Hz}, 1 \mathrm{H}), 7.18(\mathrm{~d}, J=7.8 \mathrm{~Hz}, 1 \mathrm{H}), 6.56(\mathrm{~s}, 1 \mathrm{H}), 6.21(\mathrm{~d}, J=6.6 \mathrm{~Hz}, 1 \mathrm{H}), 5.87(\mathrm{~d}, J=$ $6.6 \mathrm{~Hz}, 1 \mathrm{H}), 2.16$ (s, $3 \mathrm{H}), 2.14$ (s, $3 \mathrm{H}), 2.10$ (s, $3 \mathrm{H}), 2.03$ (s, $3 \mathrm{H}), 1.59 \mathrm{ppm}(\mathrm{s}, 3 \mathrm{H}) ;{ }^{13} \mathrm{C}$ NMR (125 MHz, $\left.\mathrm{CDCl}_{3}\right): \delta=184.1,178.2,170.7,170.4,170.1,169.8,162.1,136.2,134.2$, 132.5, 129.4, 128.9, 126.0, 123.8, 119.9, 115.6, 96.1, 81.1, 73.3, 68.0, 66.9, 21.8, 21.0, 20.7, 20.7, 16.2 ppm; HRMS (ESI-TOF): calcd for $\mathrm{C}_{26} \mathrm{H}_{22} \mathrm{~N}_{2} \mathrm{O}_{11} \mathrm{Na}\left[\mathrm{M}+\mathrm{Na}^{+}\right]$: 561.1116, found 561.1119 .

\section{Kinamycin F (1e)}

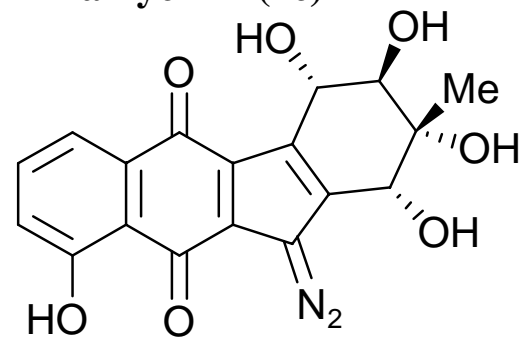

kinamycin F (1e)

To a solution of TBS-protected kinamycin C 17 (3.2 mg, 0.0052 mmol, 1.0 equiv) in THF $(0.2 \mathrm{~mL})$ at $25^{\circ} \mathrm{C}$ was added aq. $0.2 \mathrm{M}$ $\mathrm{LiOH}(0.1 \mathrm{~mL})$, and the reaction mixture was stirred at $25^{\circ} \mathrm{C}$ for $1 \mathrm{~h}$. The reaction mixture was then quenched with $\mathrm{H}_{2} \mathrm{O}(0.5$ $\mathrm{mL}$ ), and the biphasic reaction mixture was extracted with EtOAc $(3 \times 0.5 \mathrm{~mL})$, and the combined organic layers were dried $\left(\mathrm{MgSO}_{4}\right)$, and concentrated. Preparative plate chromatography (silica gel, $\mathrm{CH}_{2} \mathrm{Cl}_{2}: \mathrm{MeOH}$ 9:1) provided kinamycin F (1e, 1.8 $\mathrm{mg}, 0.0048 \mathrm{mmol}, 92 \%$ yield) as an orange foam. 1e: $\mathrm{R}_{f}=0.16$ (silica gel, $\mathrm{CH}_{2} \mathrm{Cl}_{2}: \mathrm{MeOH} 9: 1$ ); $[\alpha]_{\mathrm{D}}{ }^{32}=-103.7(\mathrm{MeCN}, c=0.08)$; IR (film) $v_{\max } 3369,2925,2855,2138,1618,1481,1455$, 1333, 1233, 1157, 1085, 1044, 1016, 971, 915, 882, 832, 771, 753, 743, $699 \mathrm{~cm}^{-1} ;{ }^{1} \mathrm{H}$ NMR (600 $\mathrm{MHz}, \mathrm{CDCl}_{3}$ ): $\delta=12.07$ (br s, $\left.1 \mathrm{H}\right), 7.45$ (t, $J=7.2 \mathrm{~Hz}, 1 \mathrm{H}$ ), 7.42 (d, $\left.J=7.2 \mathrm{~Hz}, 1 \mathrm{H}\right), 7.02$ (d, $J$ $=7.2 \mathrm{~Hz}, 1 \mathrm{H}), 4.47(\mathrm{~s}, 2 \mathrm{H}), 4.30(\mathrm{~s}, 1 \mathrm{H}), 3.69(\mathrm{~s}, 1 \mathrm{H}), 3.52(\mathrm{br} \mathrm{s}, 2 \mathrm{H}), 3.72($ br s, $1 \mathrm{H}), 1.07$ ppm (s, $3 \mathrm{H}) ;{ }^{13} \mathrm{C}$ NMR $\left(125 \mathrm{MHz}, \mathrm{CDCl}_{3}\right): \delta=184.5,181.3,162.8,138.8,136.9,135.3,131.9$, 130.6, 130.1, 124.7, 120.2, 116.8, 75.9, 74.9, 74.3, 69.6, 69.1, 21.5 ppm; HRMS (ESI-TOF): calcd for $\mathrm{C}_{18} \mathrm{H}_{14} \mathrm{~N}_{2} \mathrm{O}_{7} \mathrm{Na}\left[\mathrm{M}+\mathrm{Na}^{+}\right]$: 393.0693, found 393.0689. 


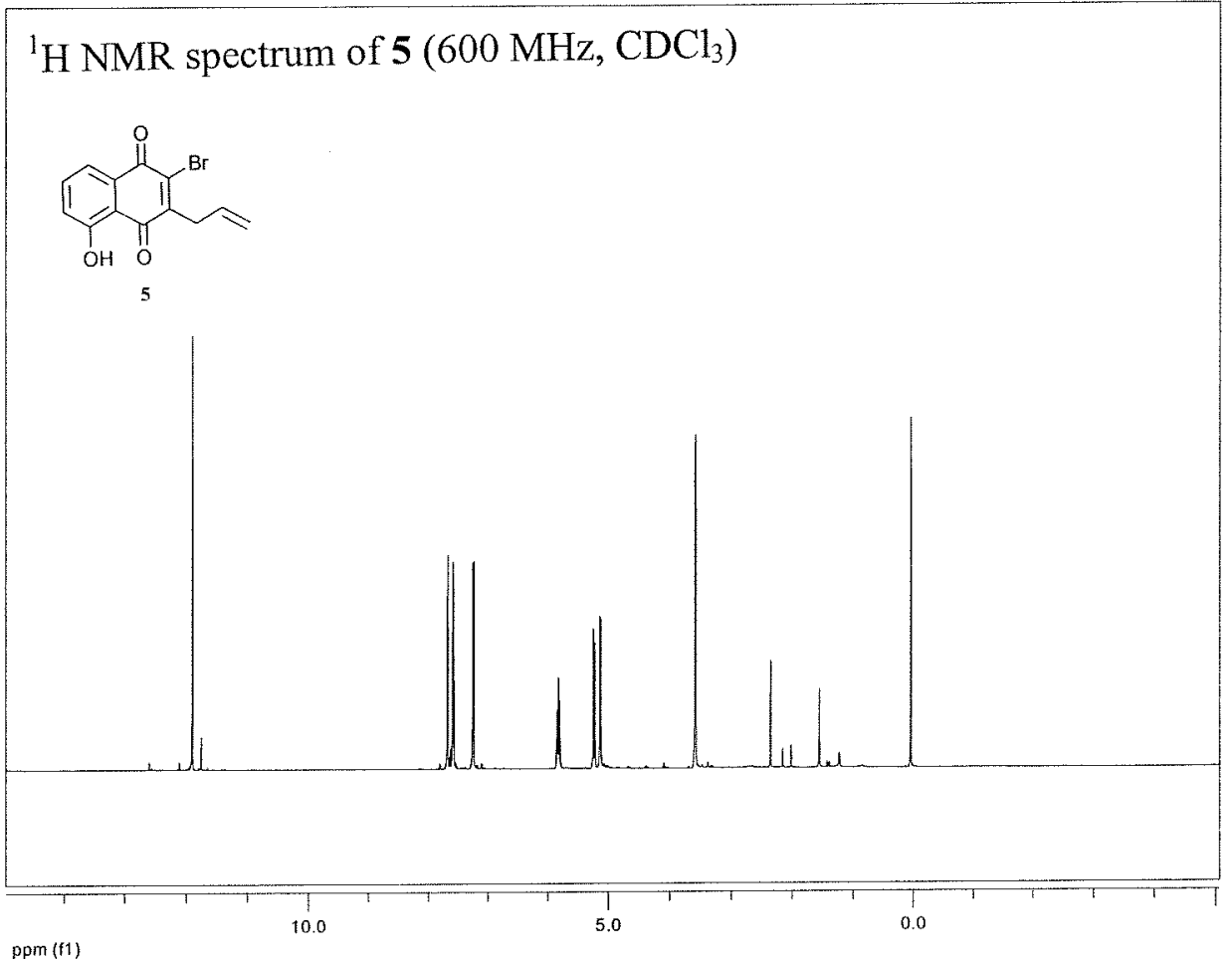

${ }^{13} \mathrm{C}$ NMR spectrum of $5\left(150 \mathrm{MHz}, \mathrm{CDCl}_{3}\right)$

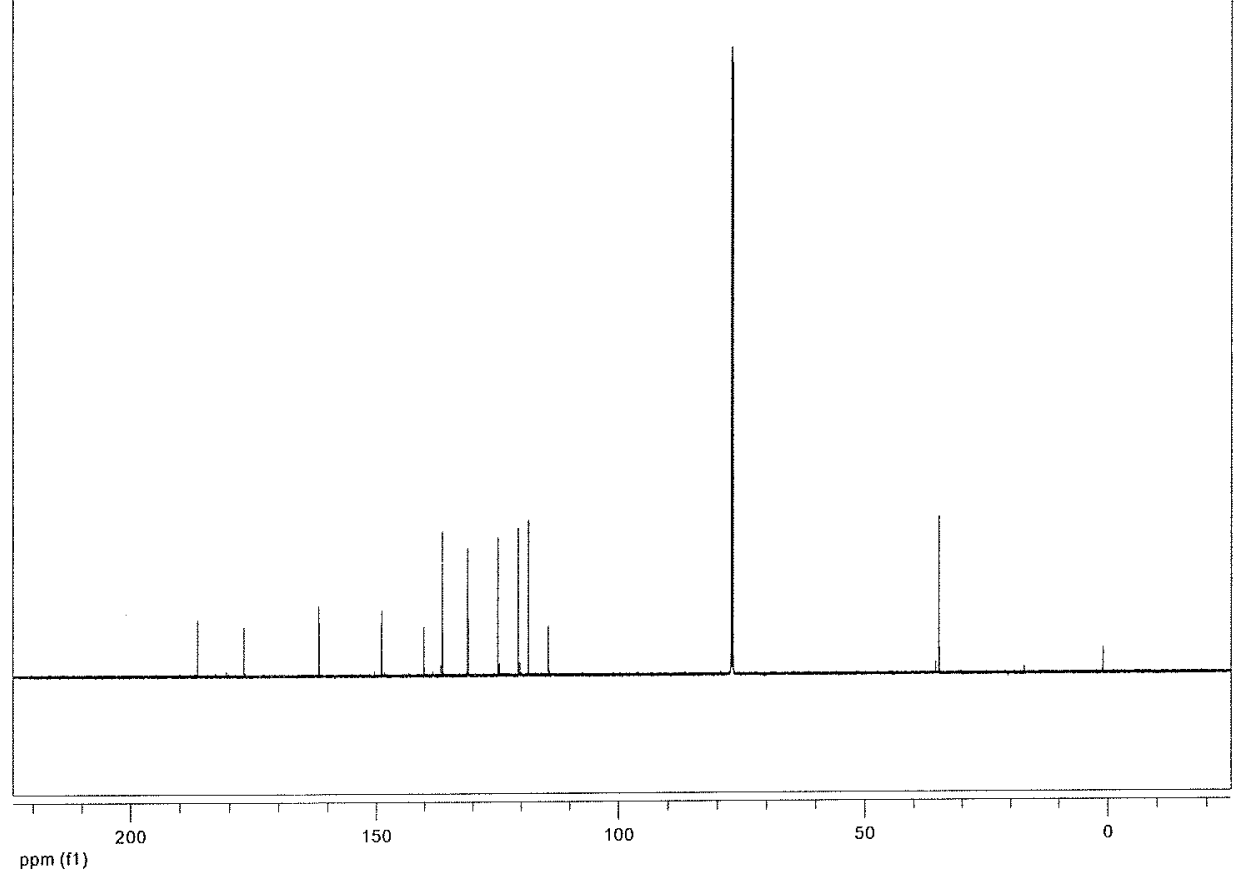




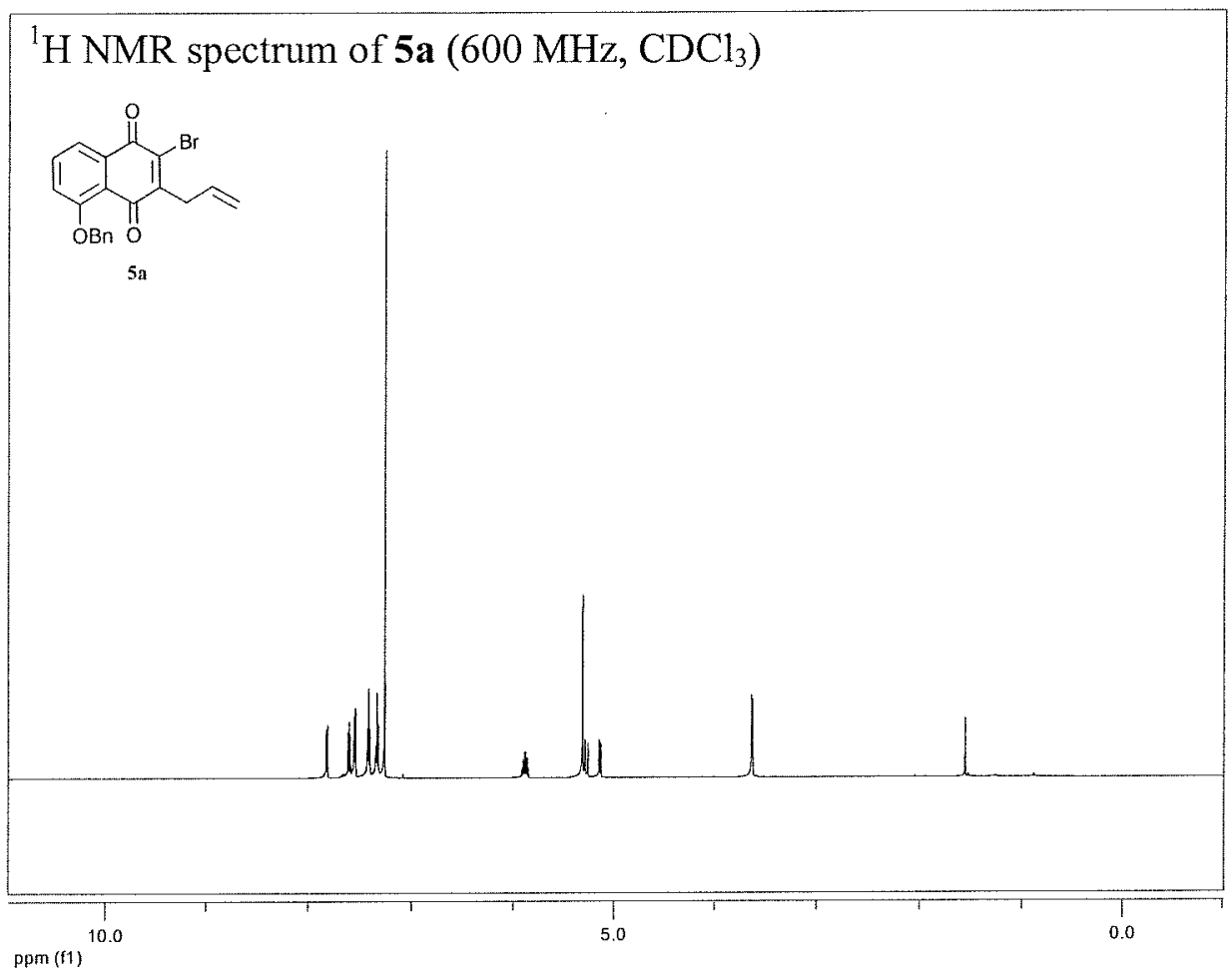

${ }^{13} \mathrm{C}$ NMR spectrum of $\mathbf{5 a}\left(150 \mathrm{MHz}, \mathrm{CDCl}_{3}\right)$

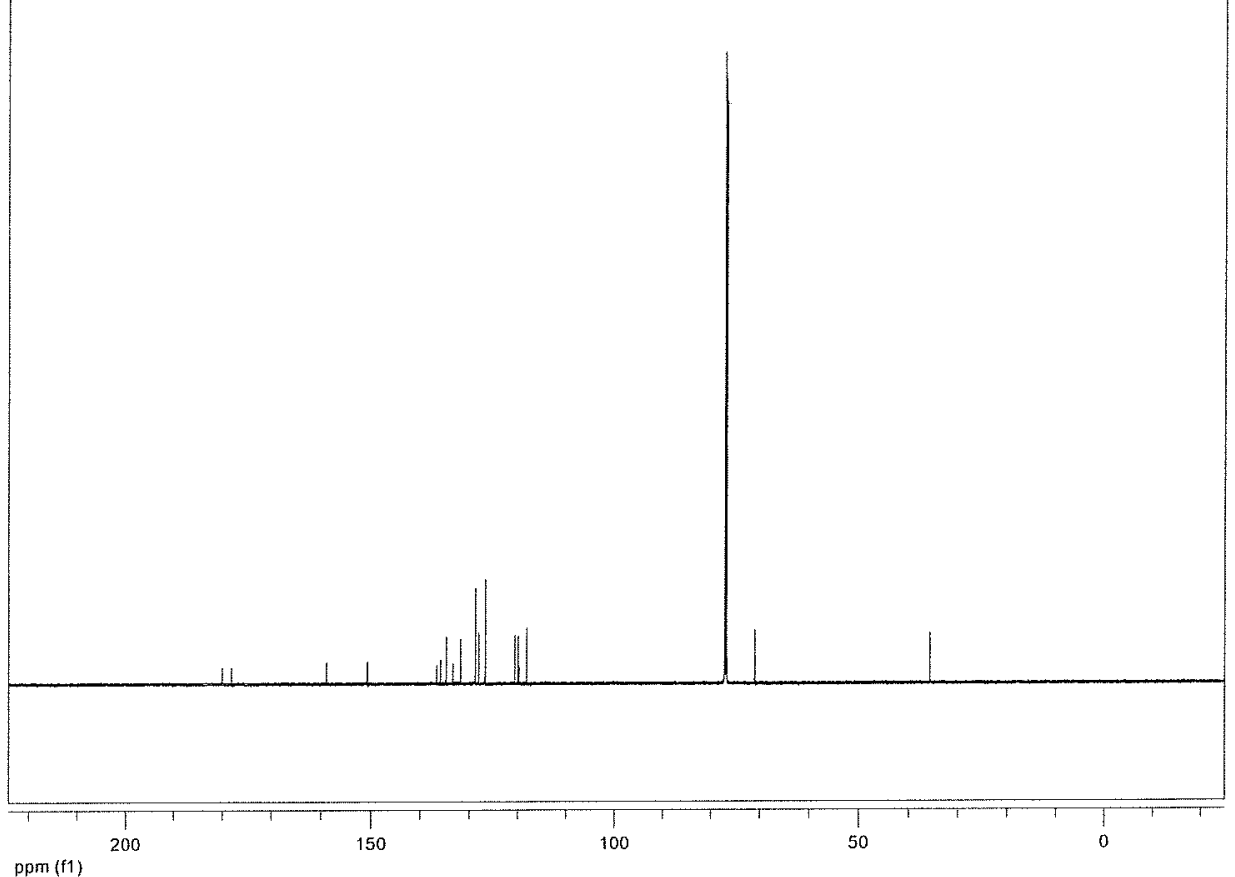




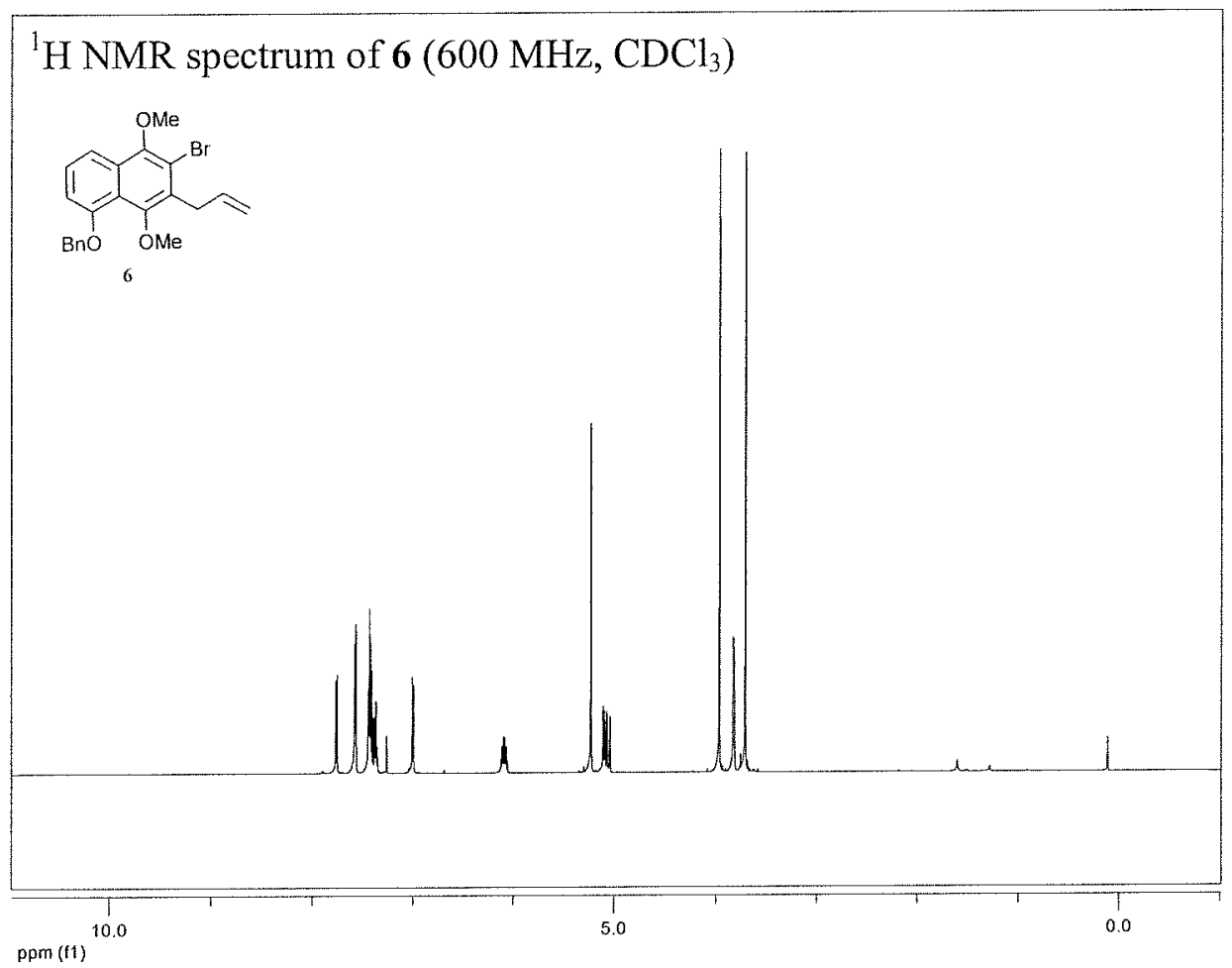

${ }^{13} \mathrm{C}$ NMR spectrum of $6\left(150 \mathrm{MHz}, \mathrm{CDCl}_{3}\right)$

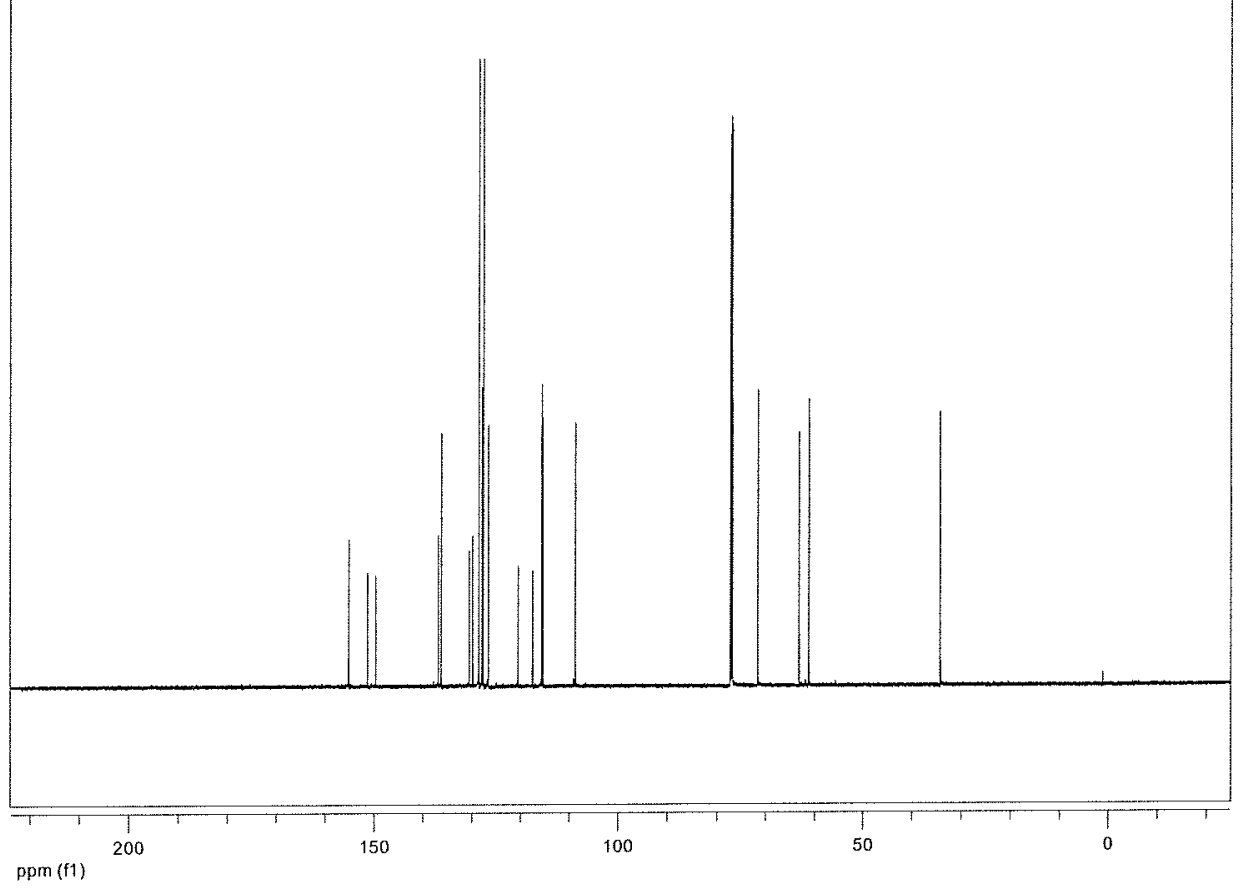




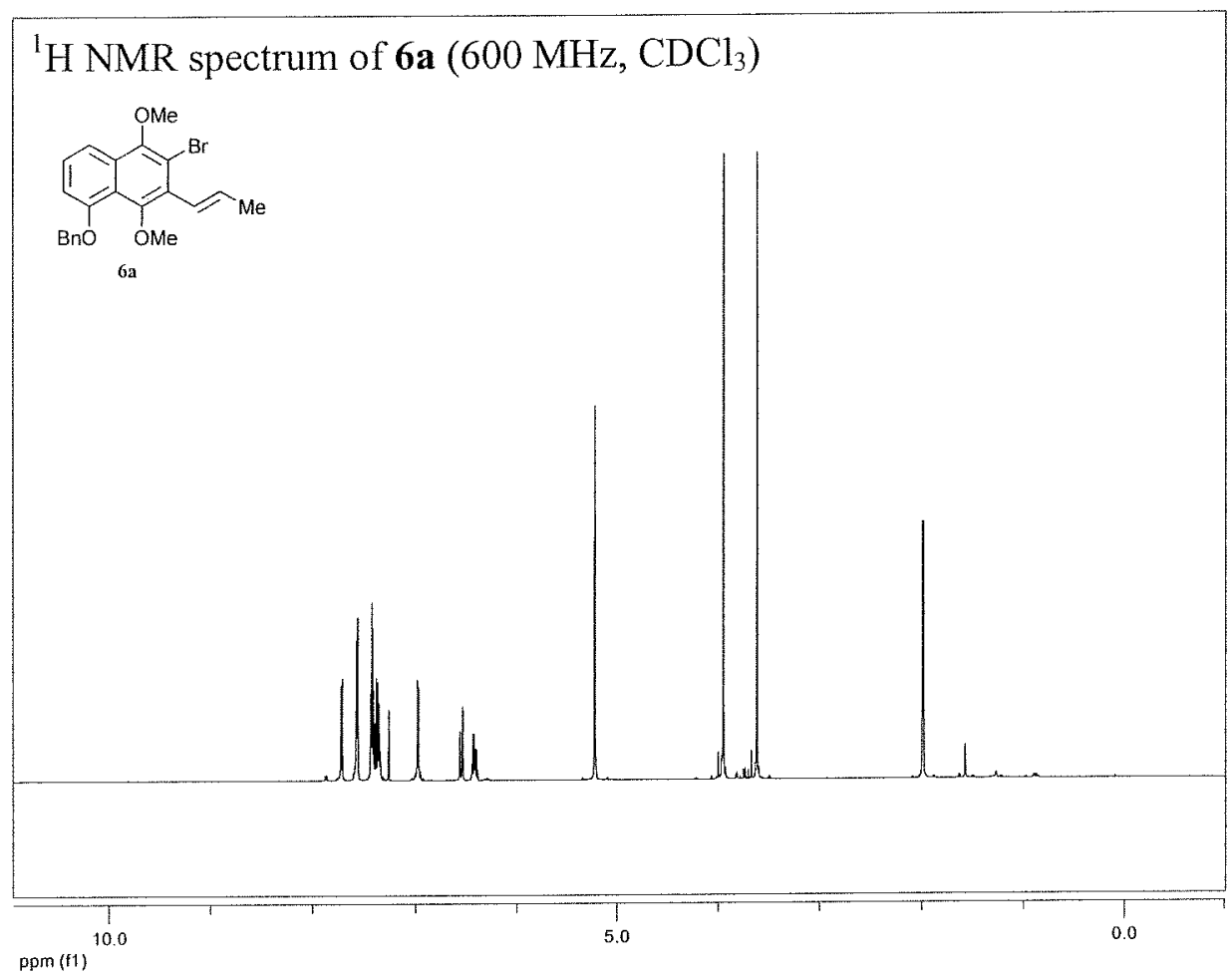

${ }^{13} \mathrm{C} \mathrm{NMR}$ spectrum of $6 \mathbf{a}\left(150 \mathrm{MHz}, \mathrm{CDCl}_{3}\right)$

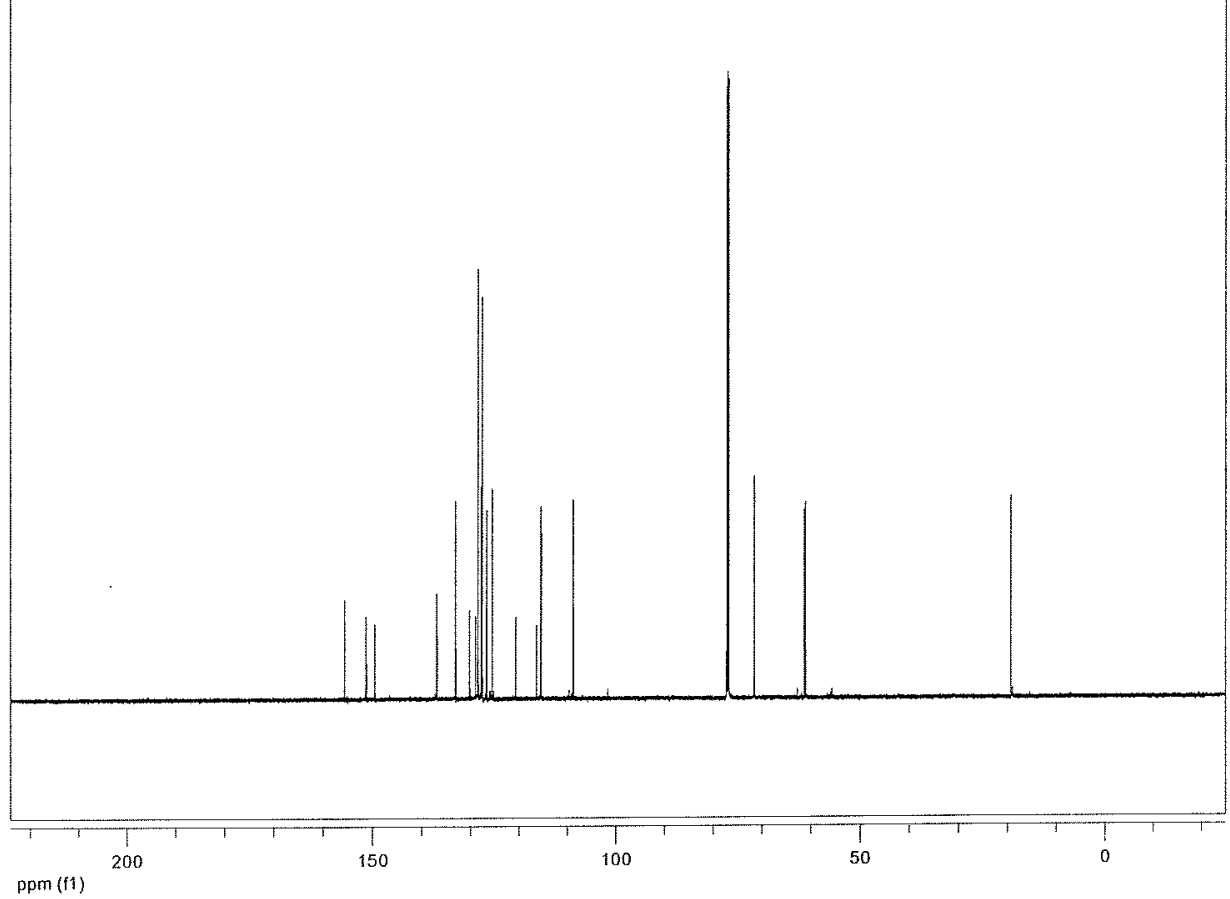




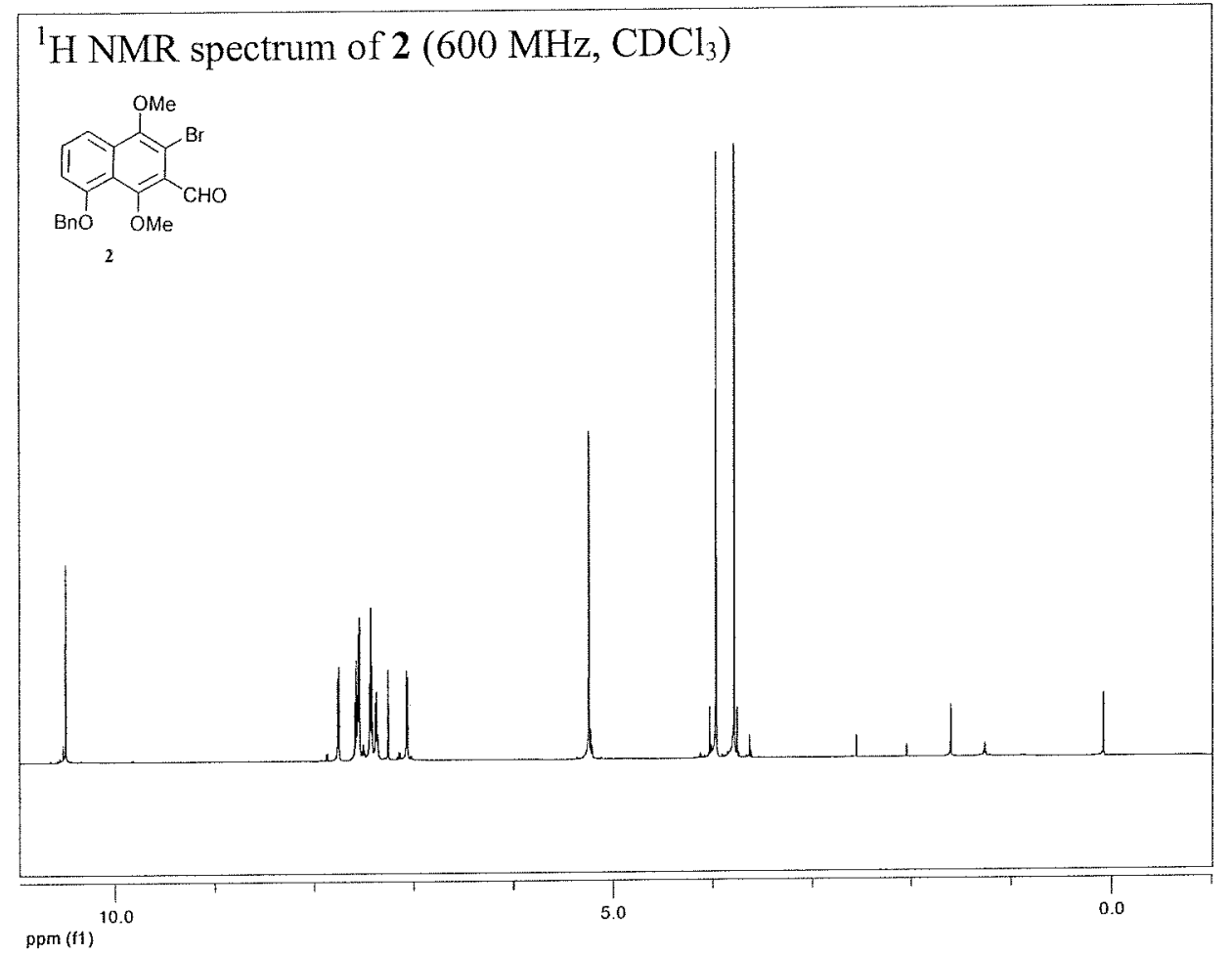

${ }^{13} \mathrm{C} \mathrm{NMR}$ spectrum of $2\left(150 \mathrm{MHz}, \mathrm{CDCl}_{3}\right)$

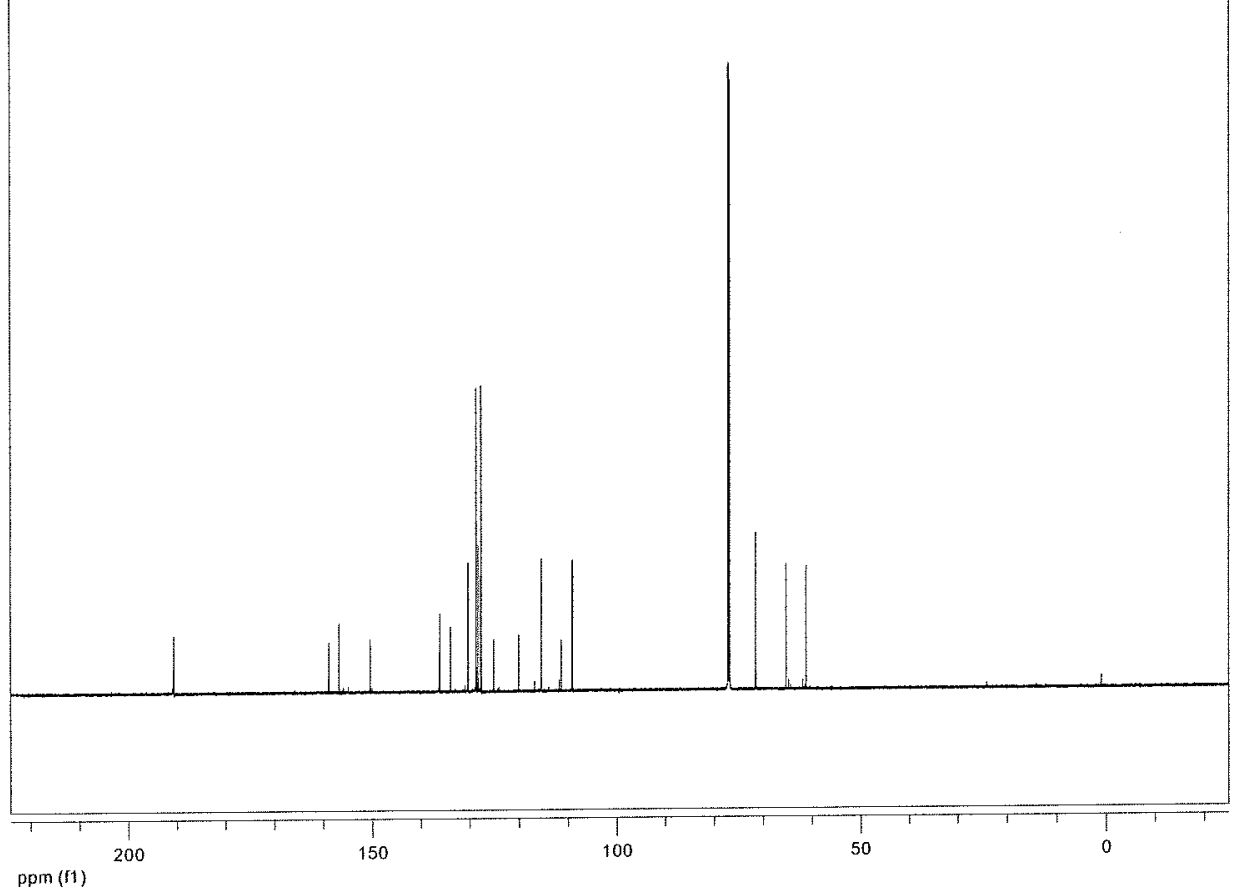




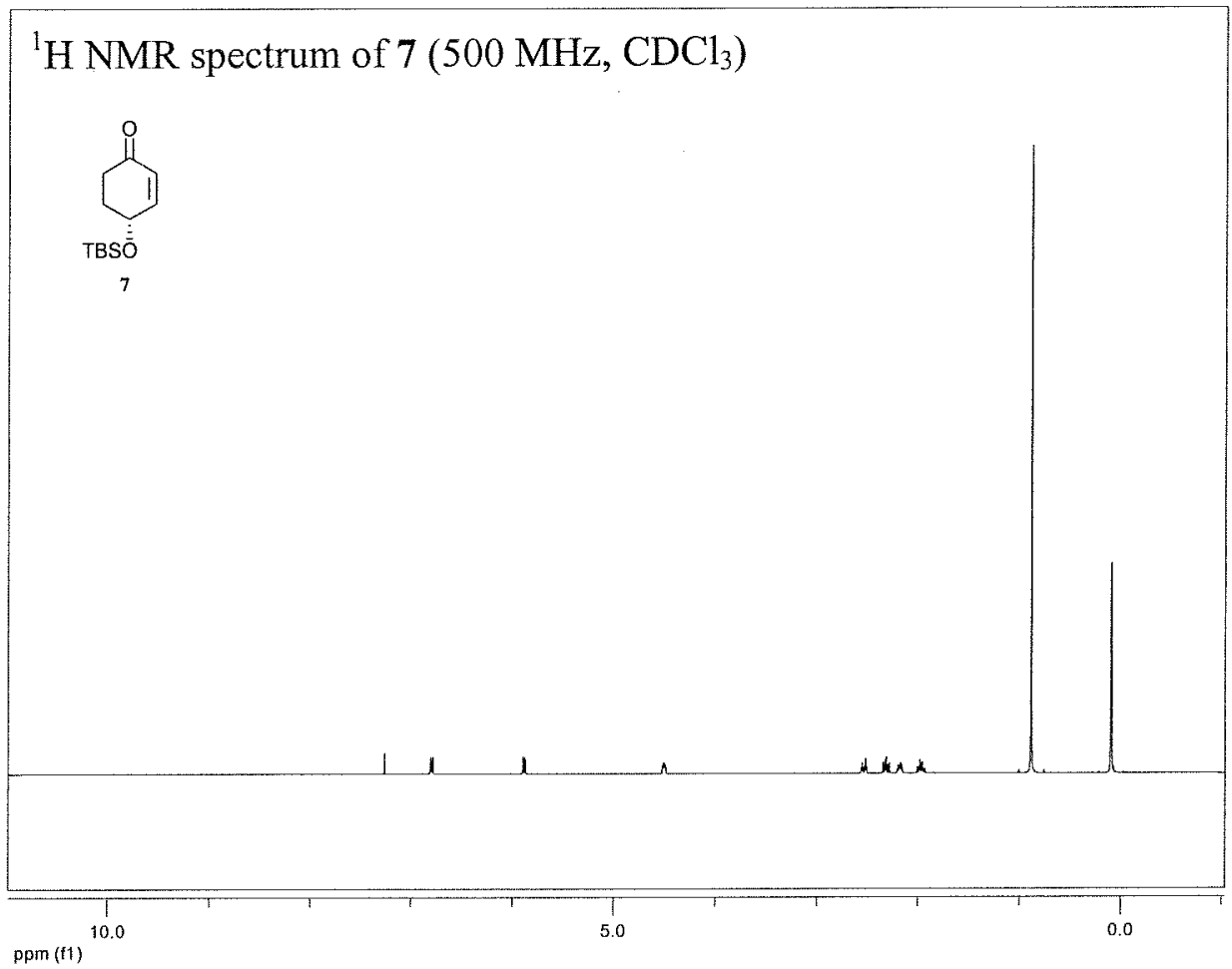

${ }^{13} \mathrm{C} \mathrm{NMR} \mathrm{spectrum} \mathrm{of} 7\left(125 \mathrm{MHz}, \mathrm{CDCl}_{3}\right)$

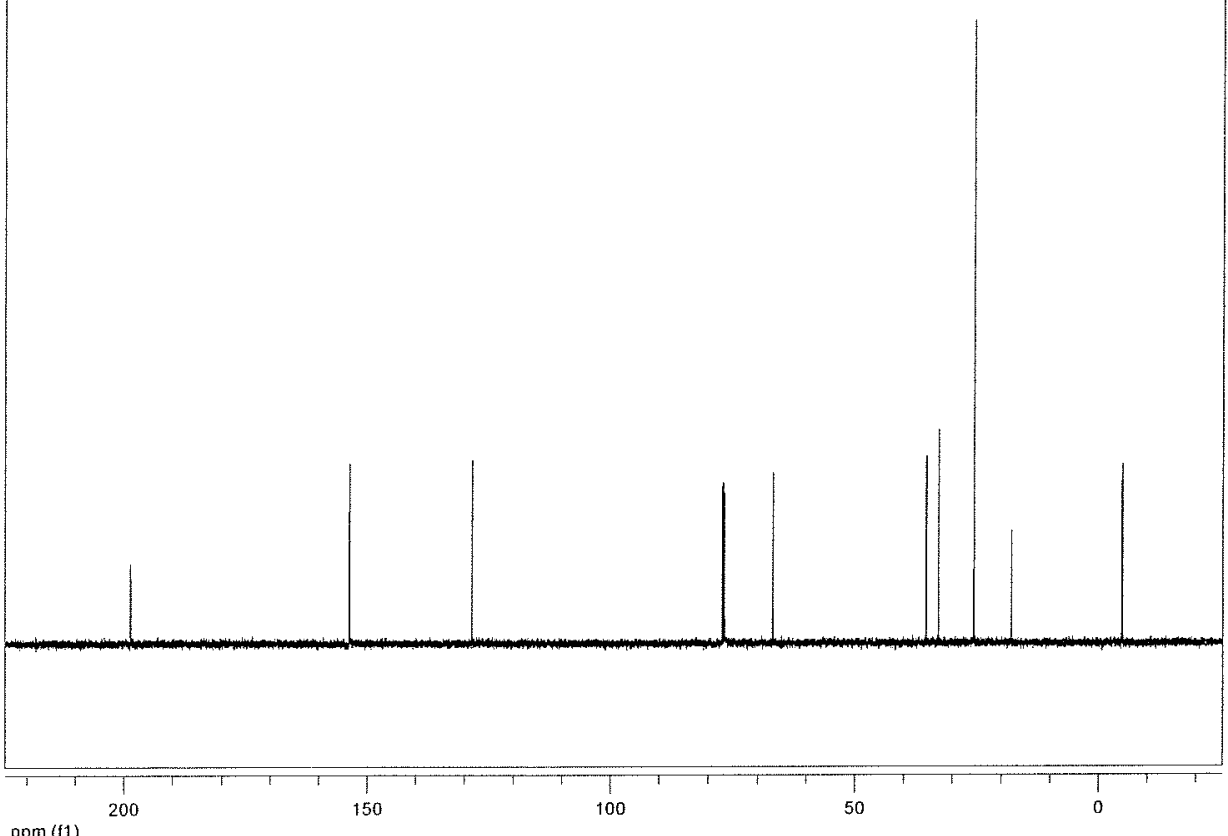




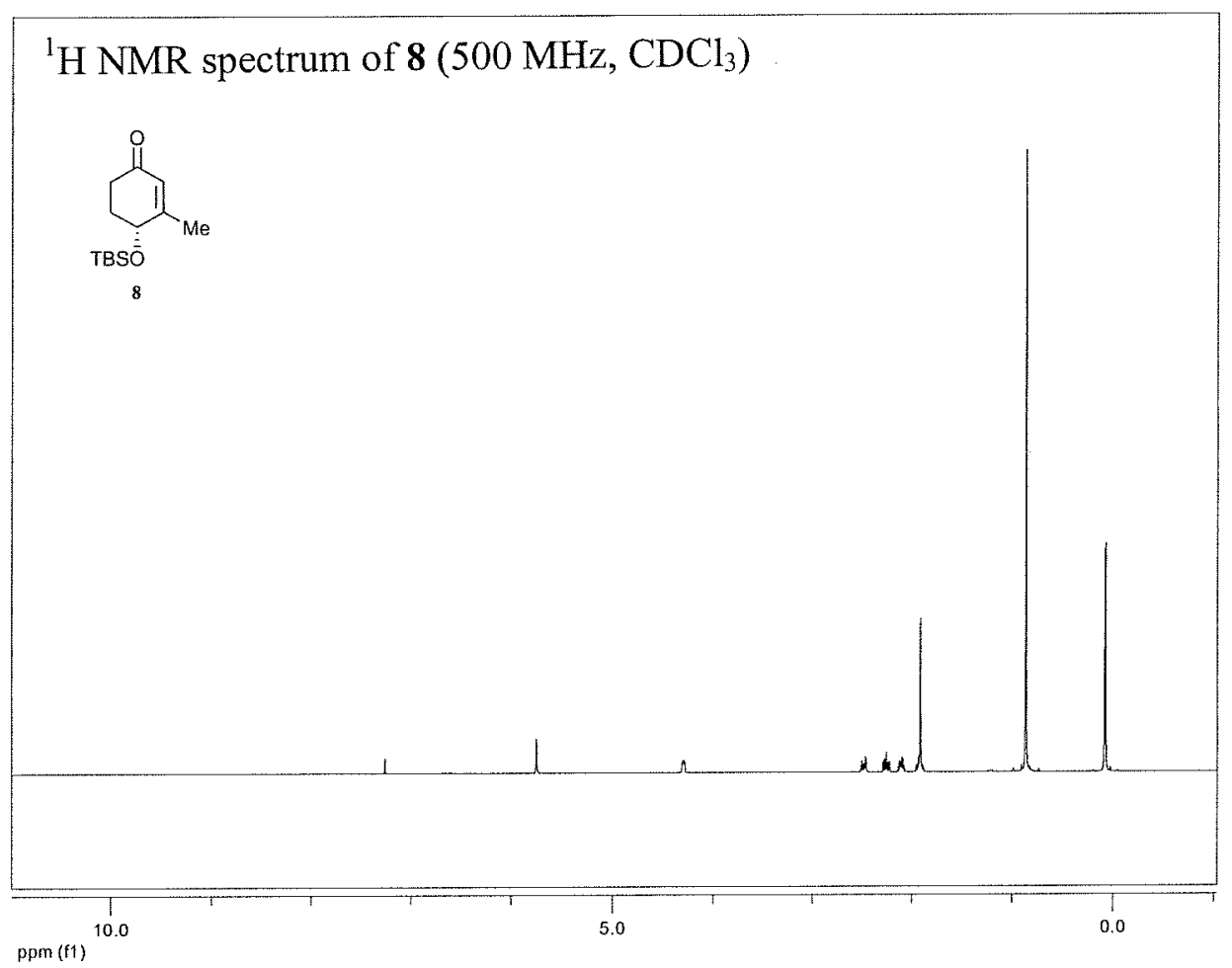

${ }^{13} \mathrm{C}$ NMR spectrum of $8\left(125 \mathrm{MHz}, \mathrm{CDCl}_{3}\right)$

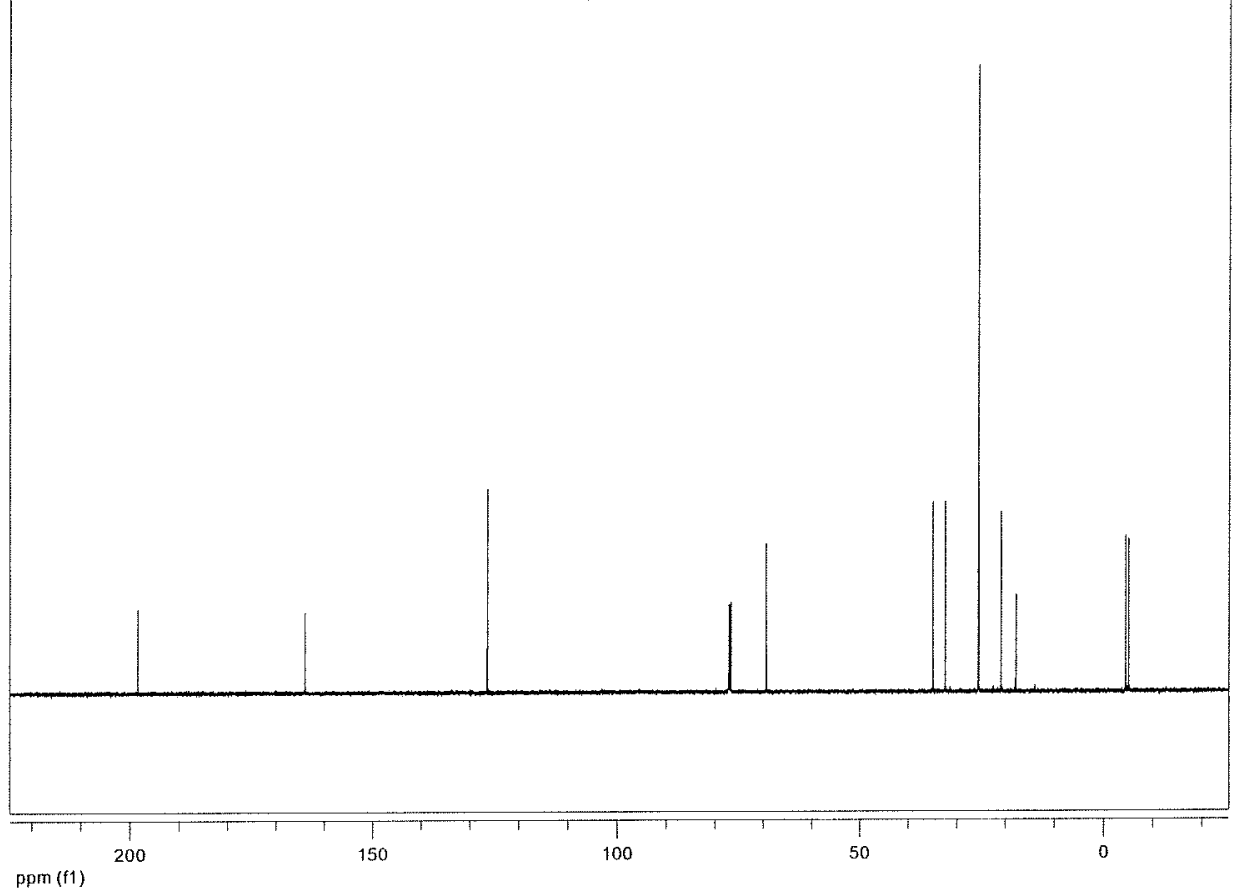




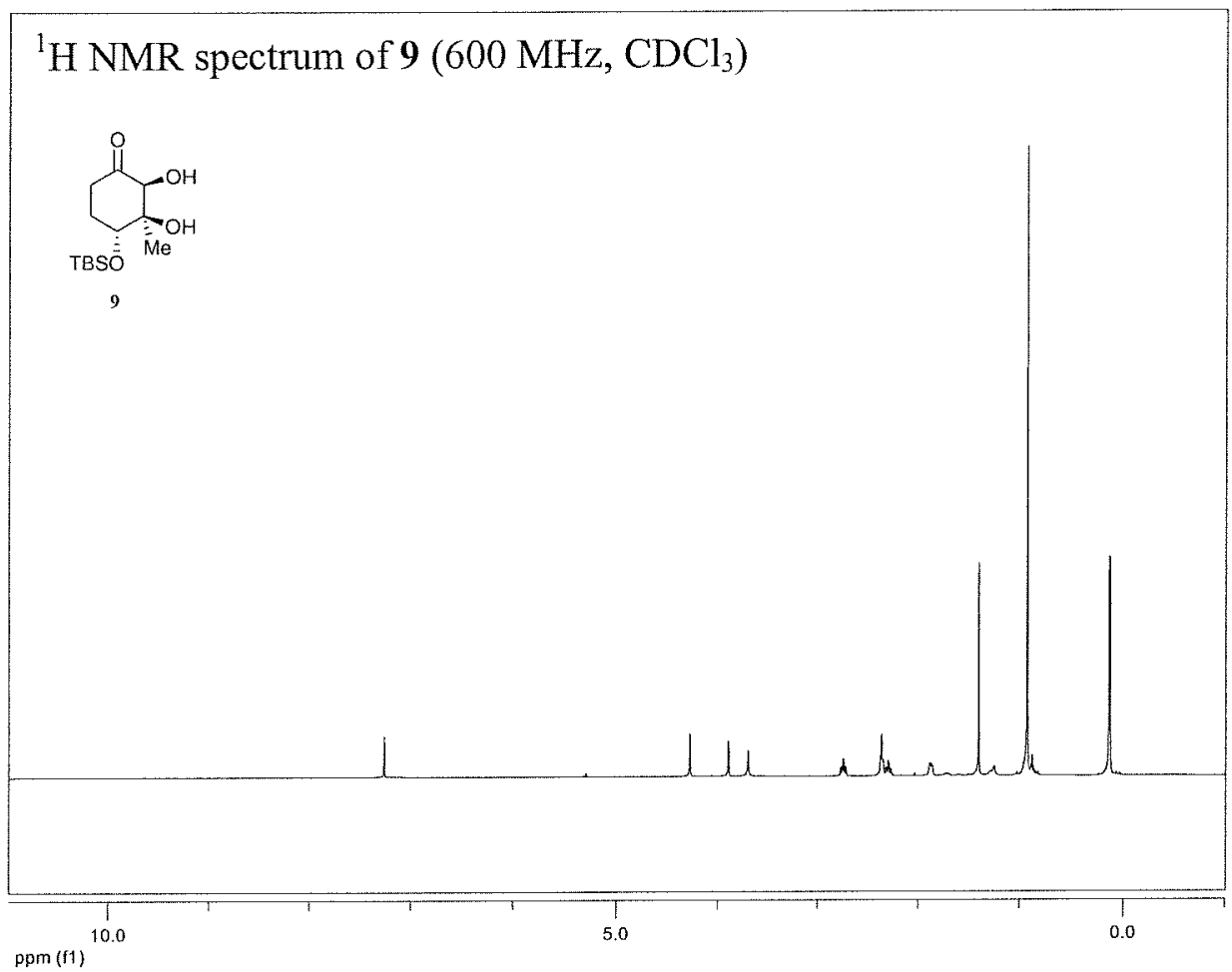

${ }^{13} \mathrm{C}$ NMR spectrum of $9\left(150 \mathrm{MHz}, \mathrm{CDCl}_{3}\right)$

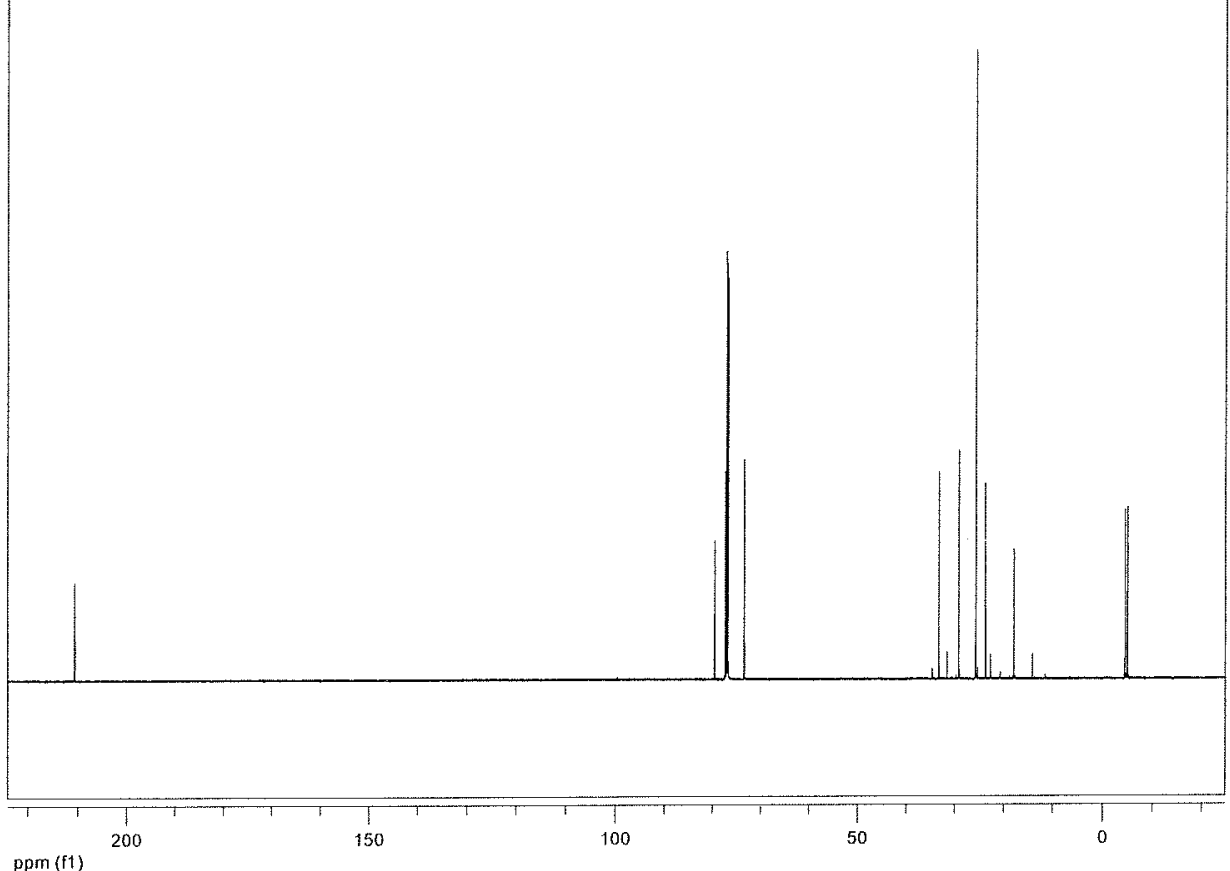



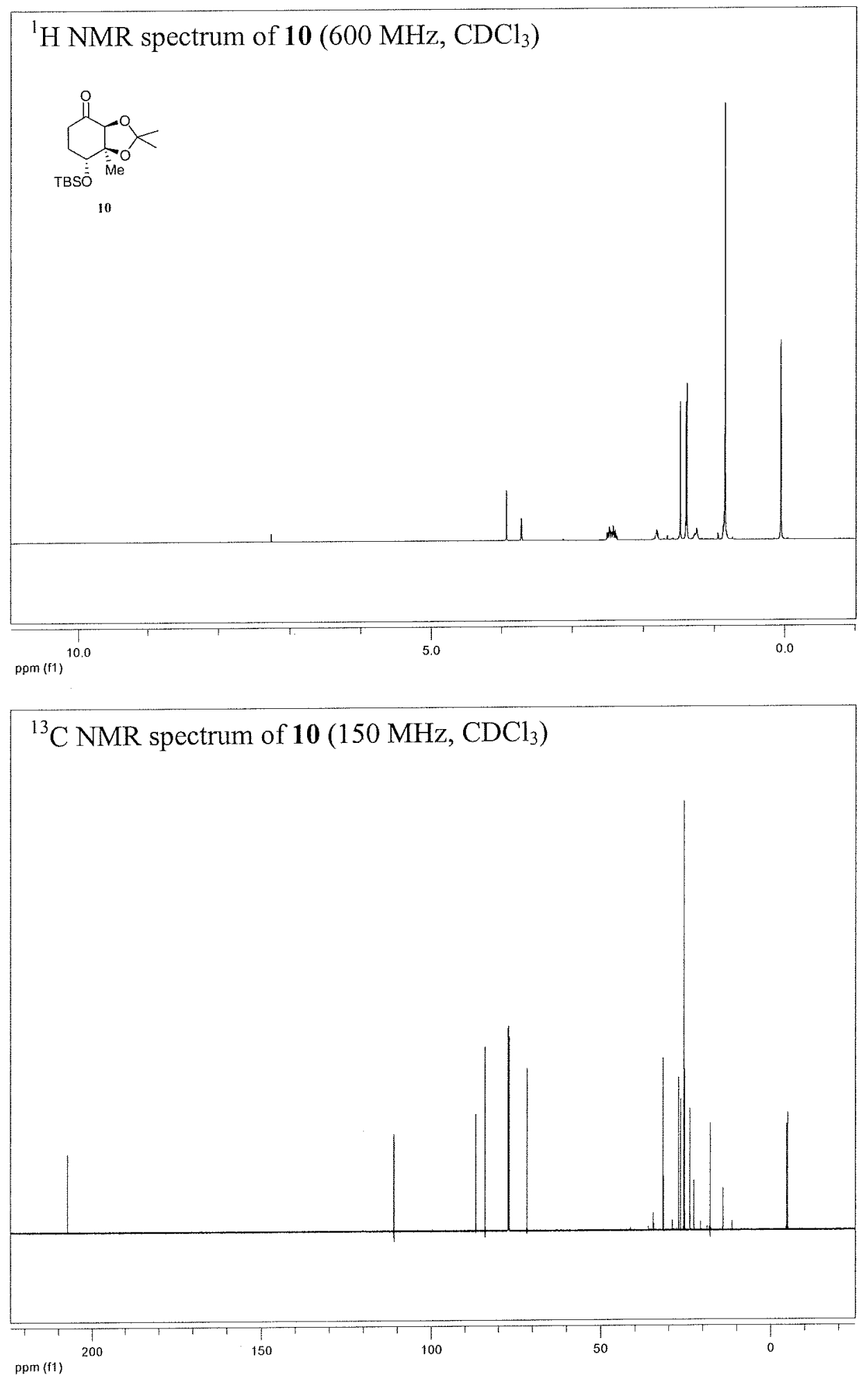


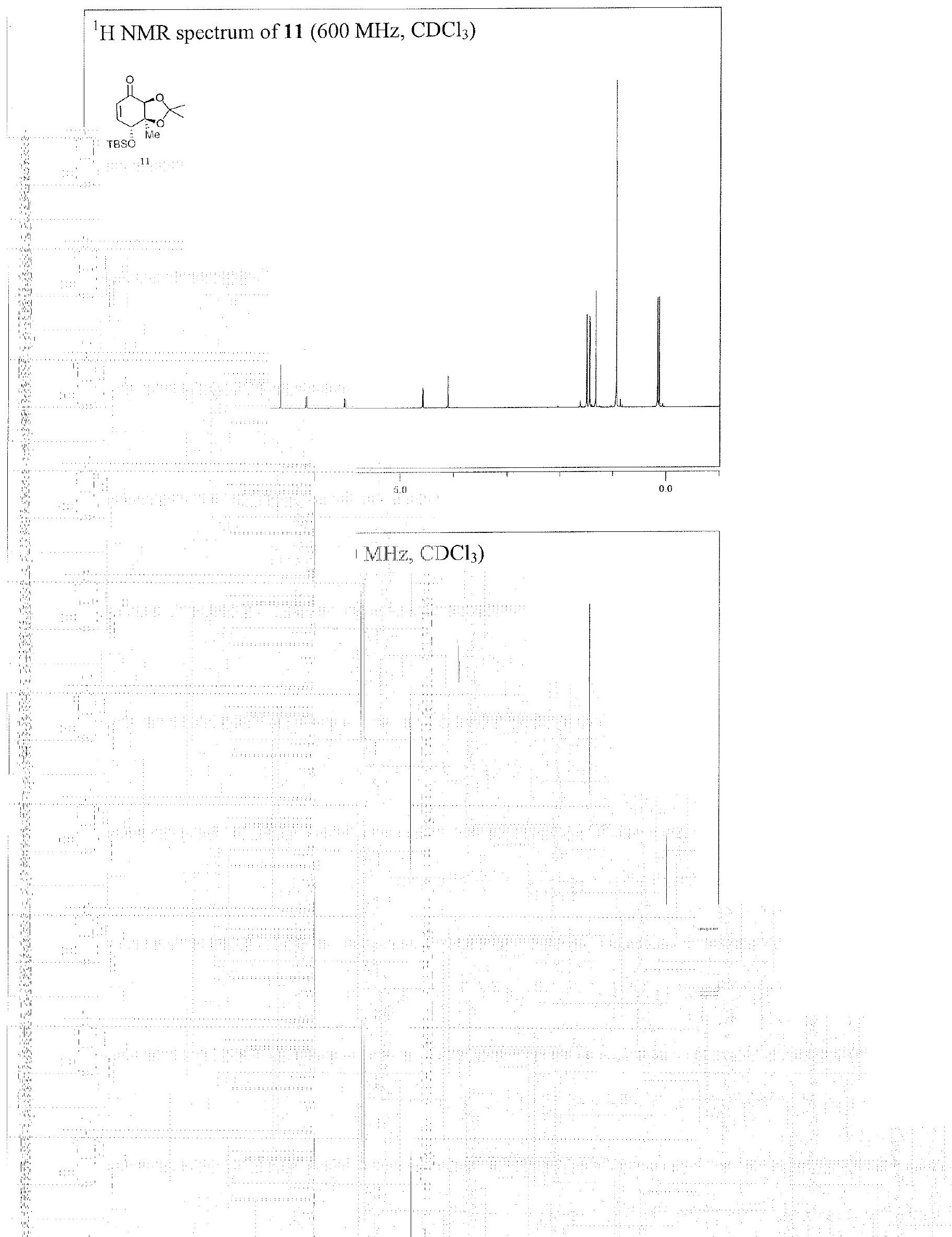




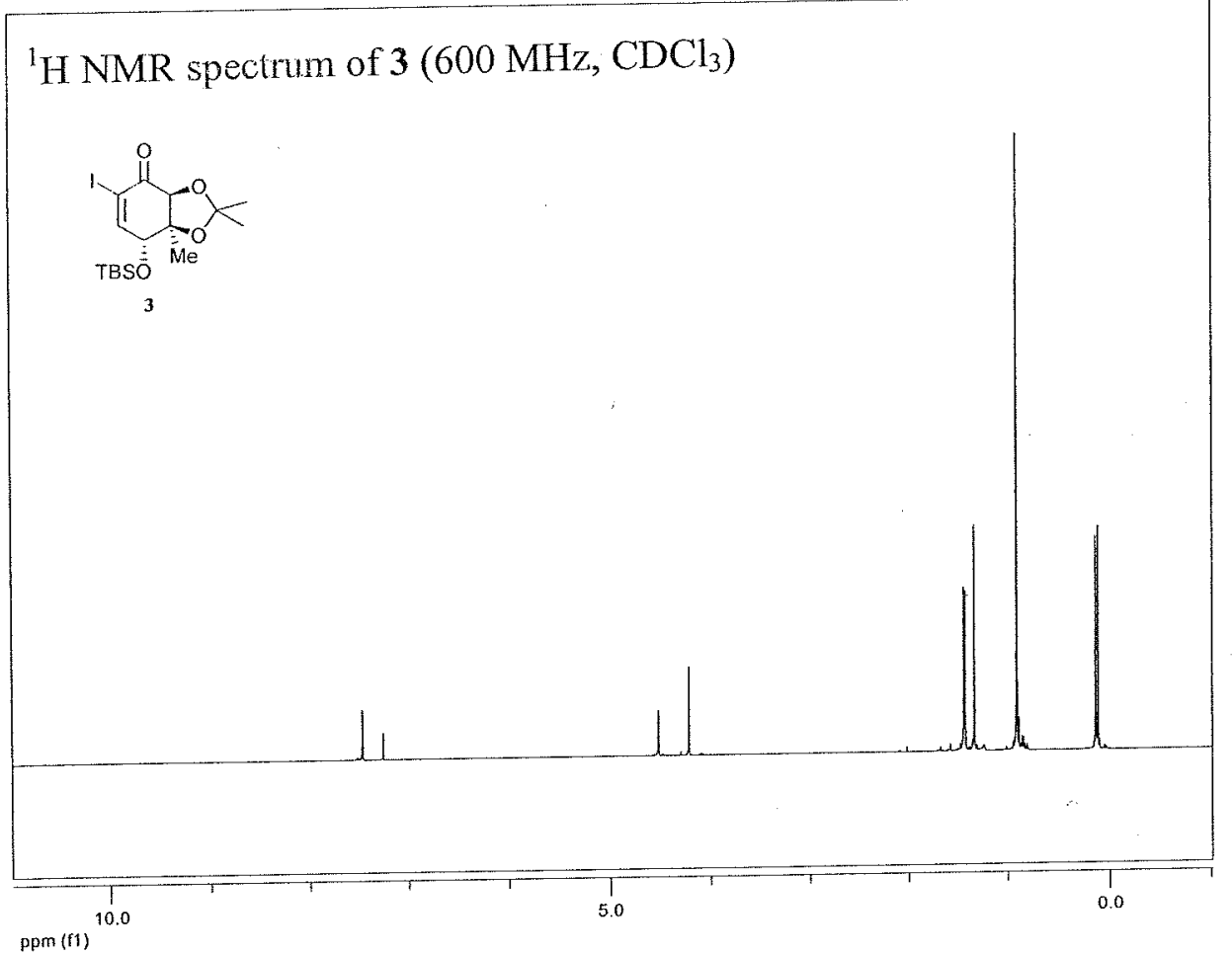

${ }^{13} \mathrm{C}$ NMR spectrum of $3\left(150 \mathrm{MHz}, \mathrm{CDCl}_{3}\right)$

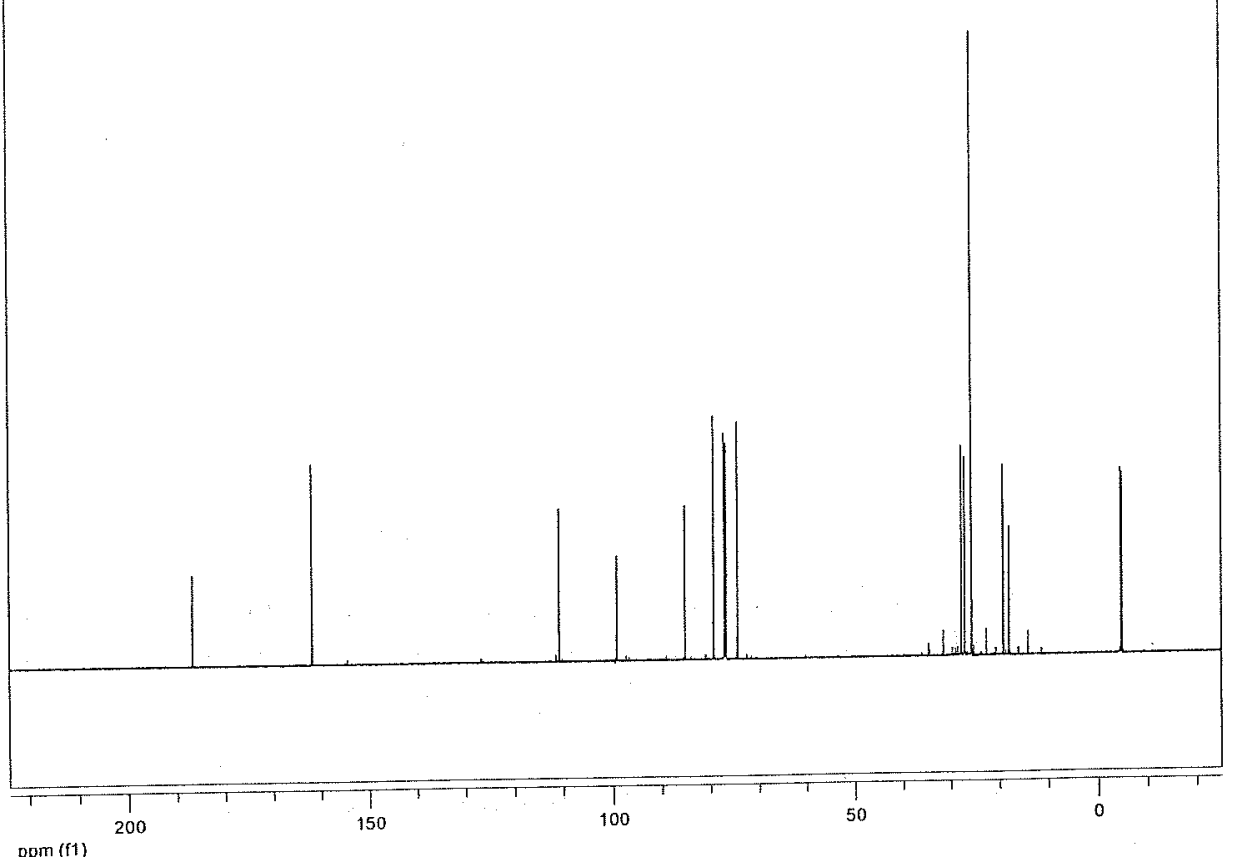




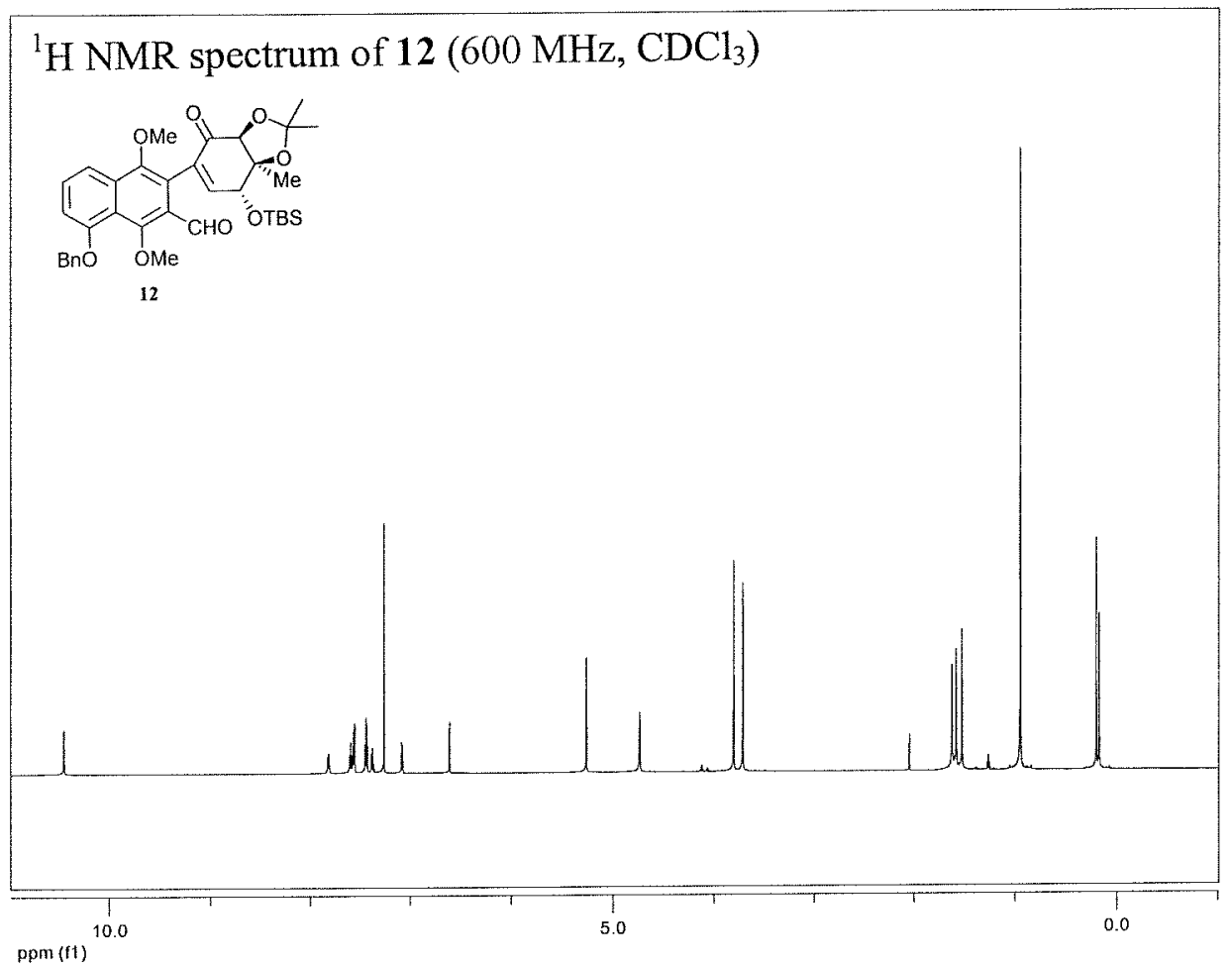

${ }^{13} \mathrm{C} \mathrm{NMR}$ spectrum of $12\left(150 \mathrm{MHz}, \mathrm{CDCl}_{3}\right)$

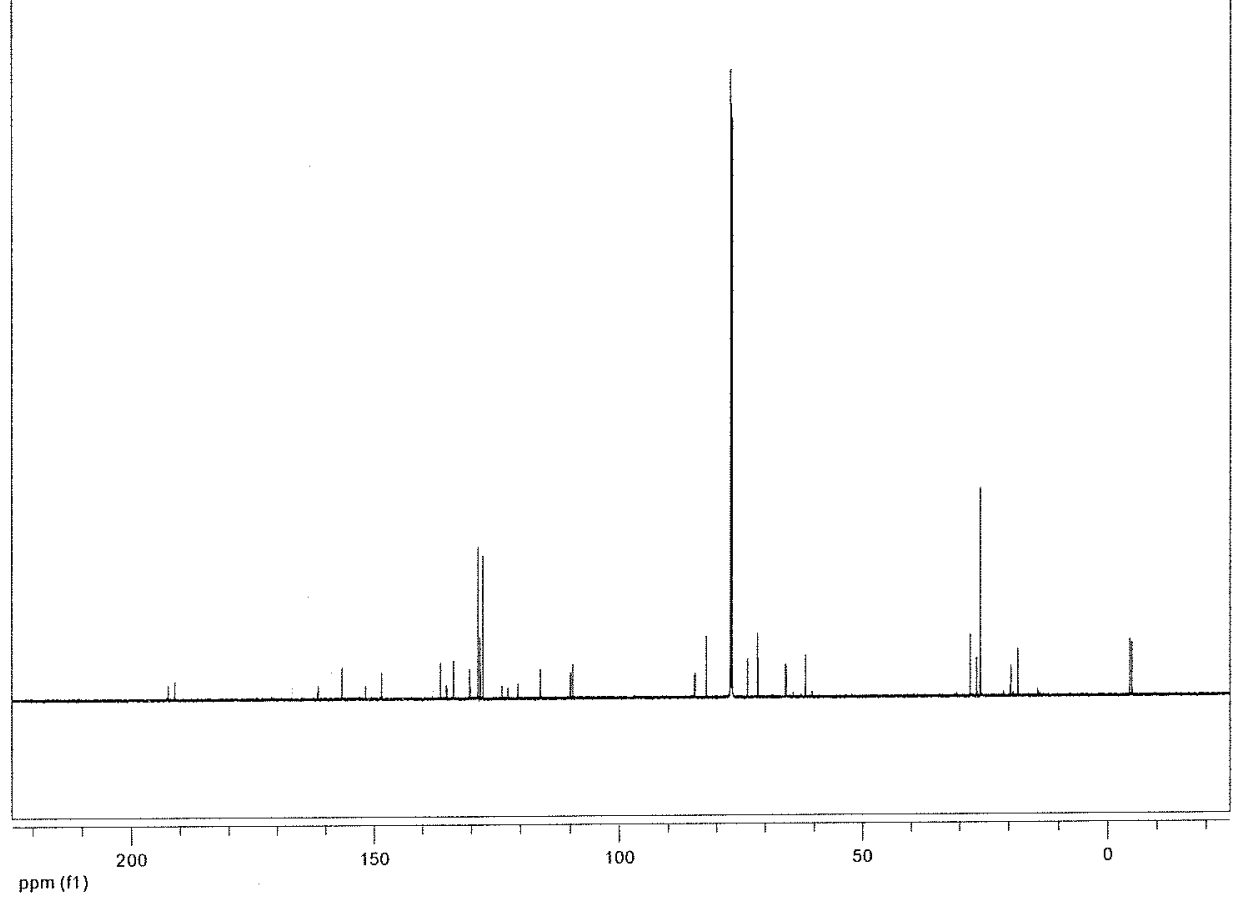




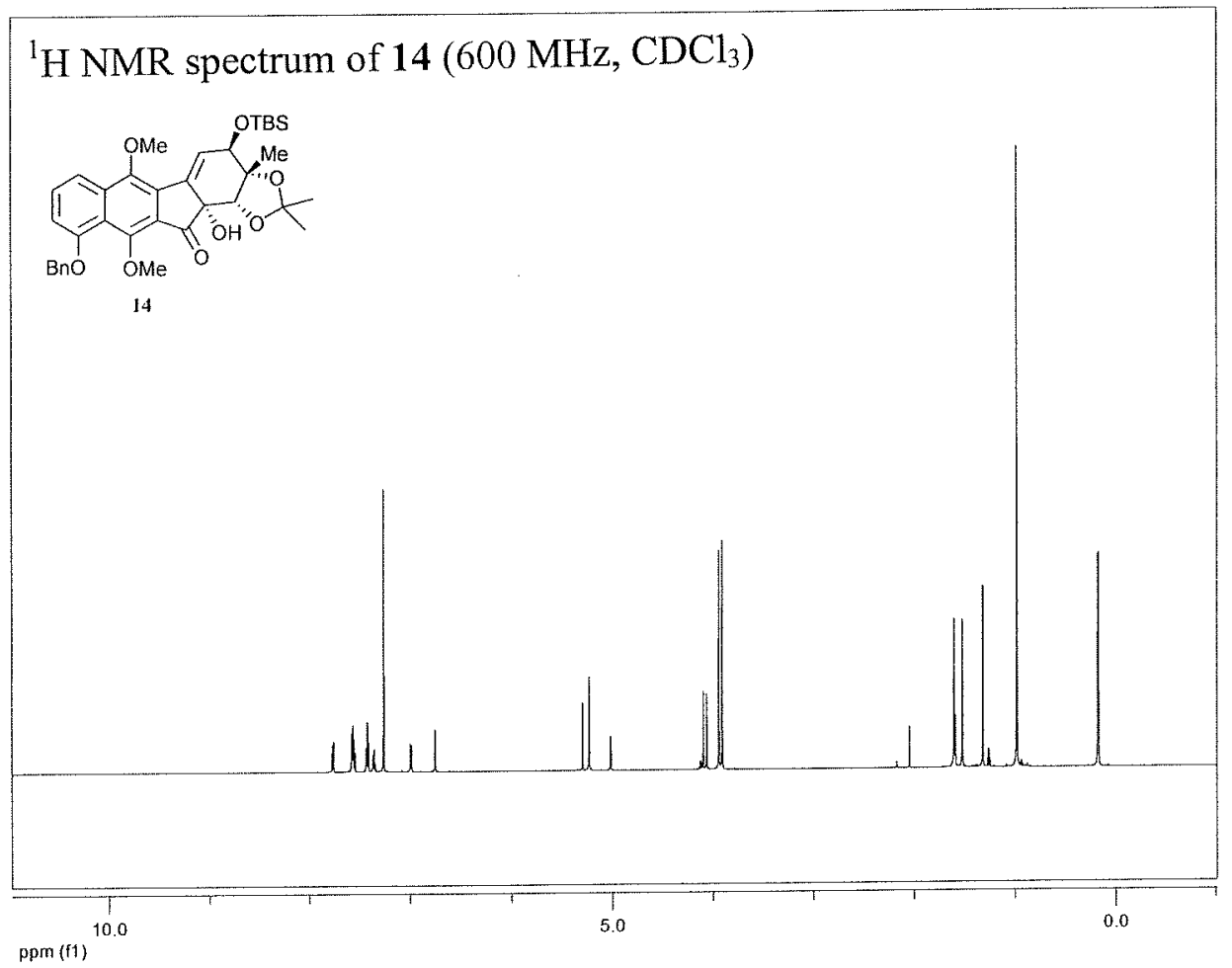

${ }^{13} \mathrm{C}$ NMR spectrum of $14\left(150 \mathrm{MHz}, \mathrm{CDCl}_{3}\right)$

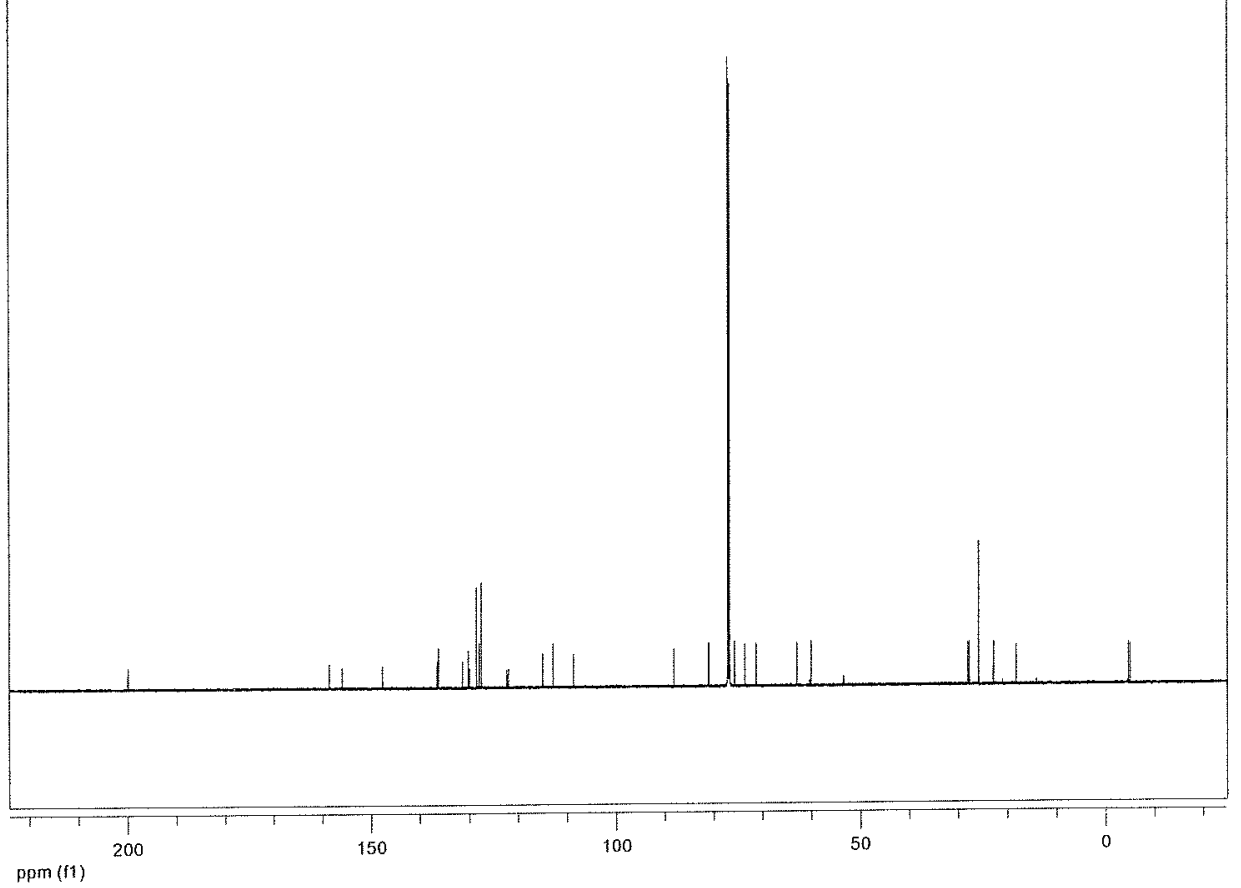



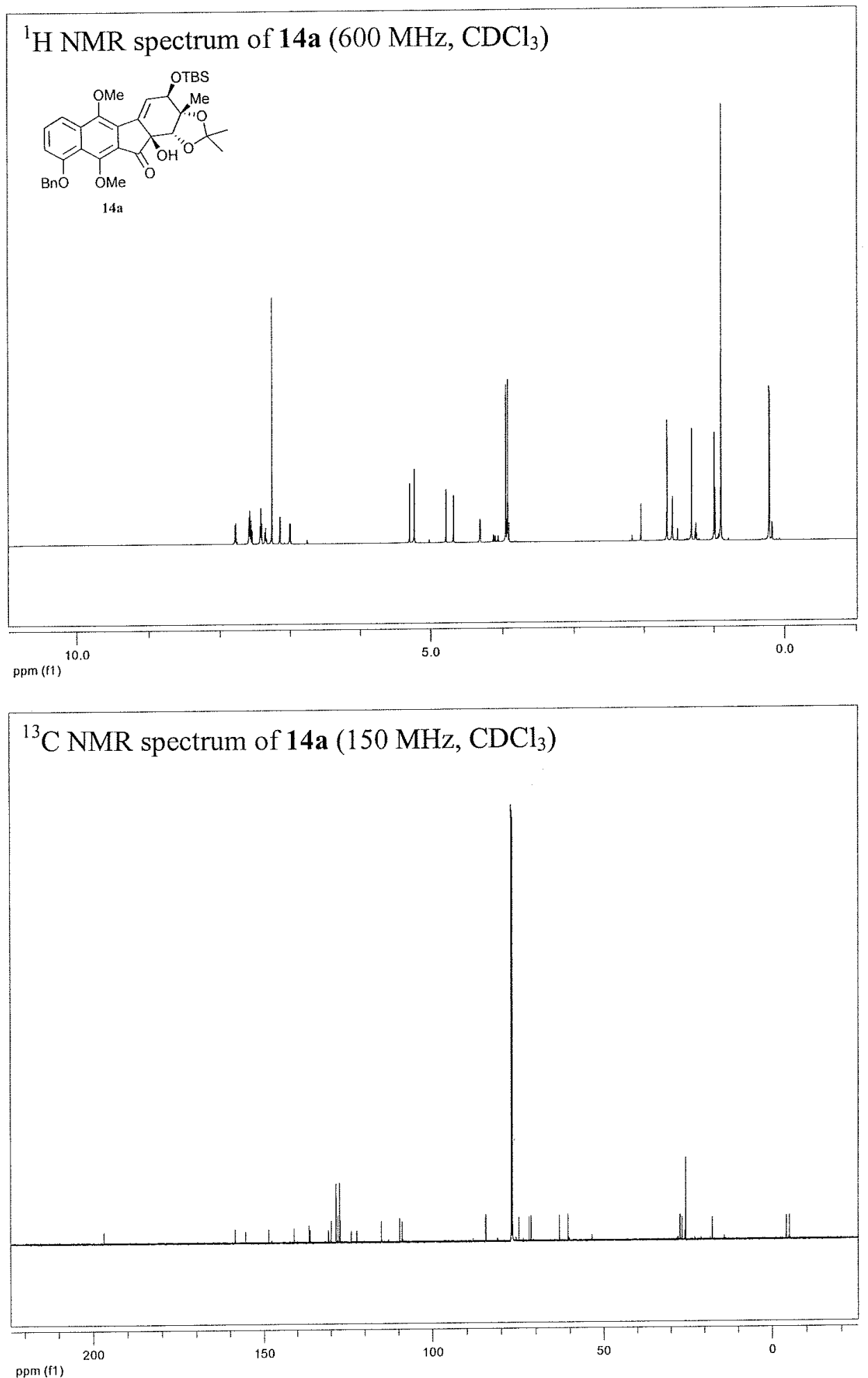

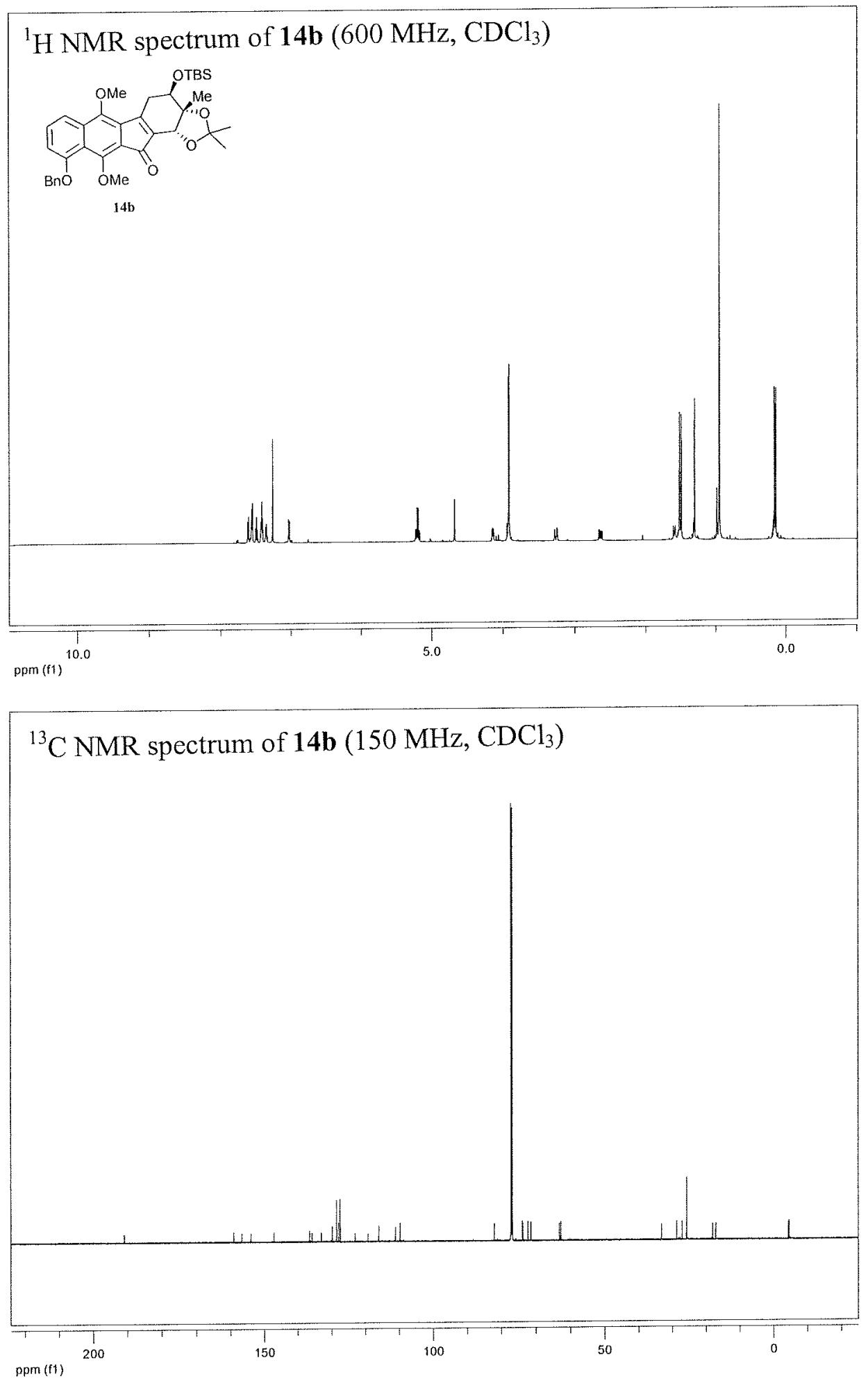


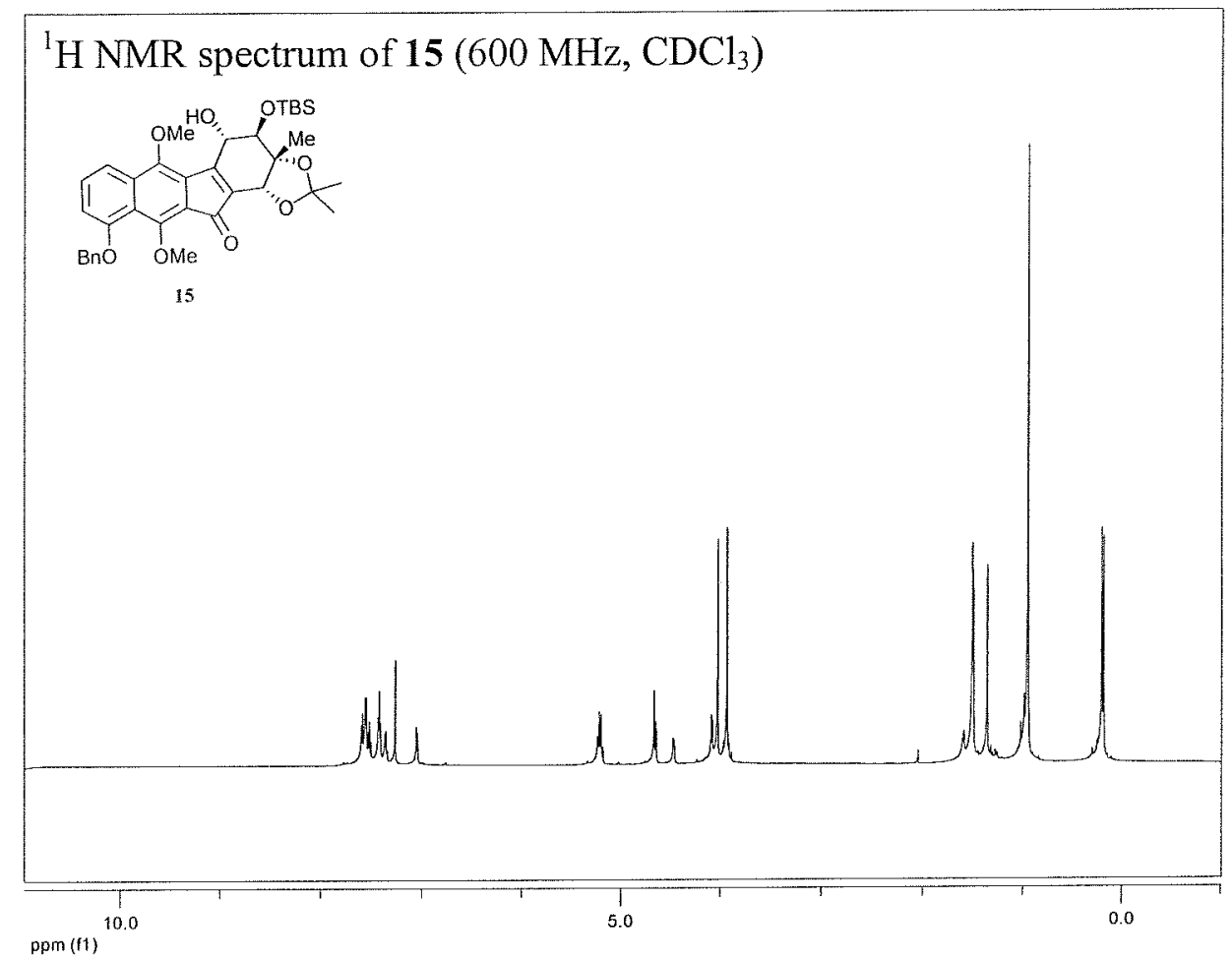

${ }^{13} \mathrm{C}$ NMR spectrum of $15\left(150 \mathrm{MHz}, \mathrm{CDCl}_{3}\right)$

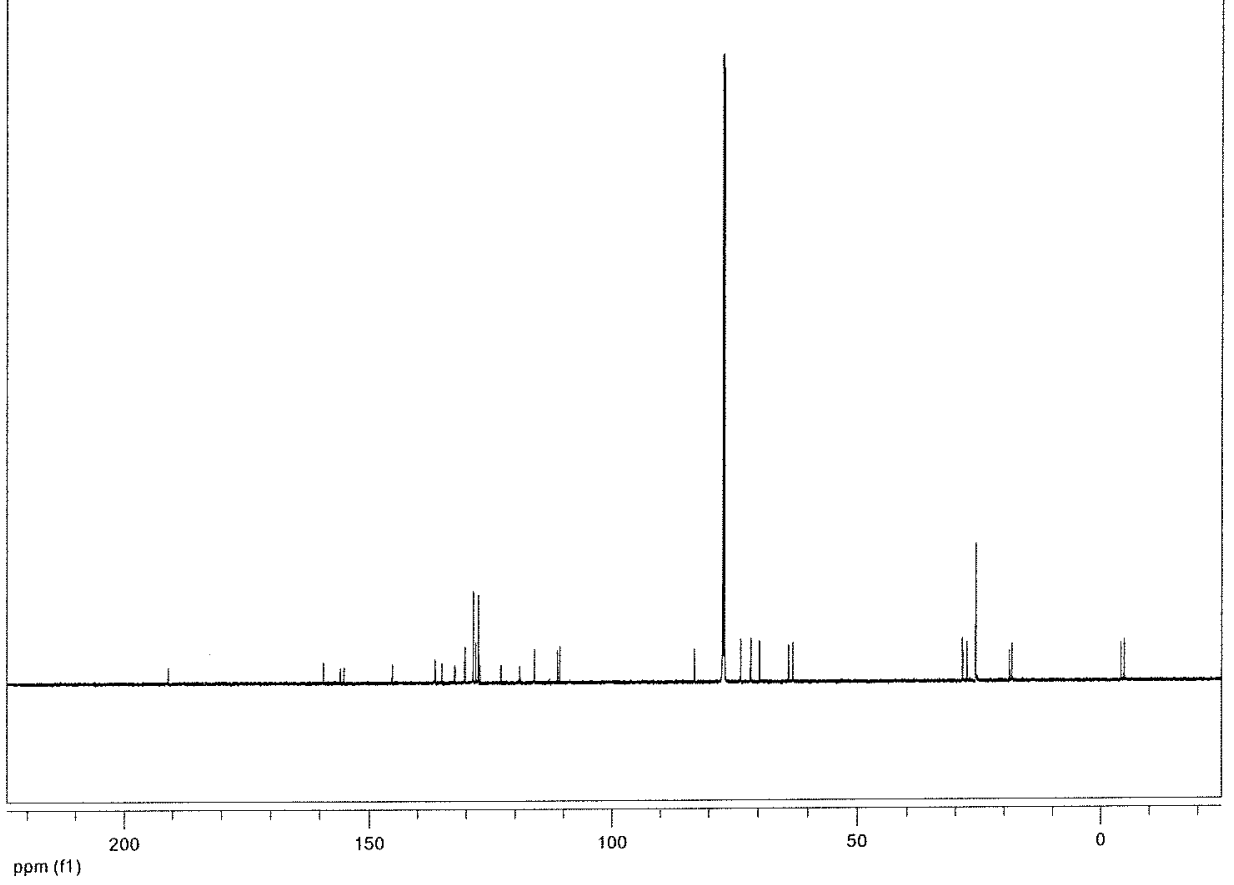




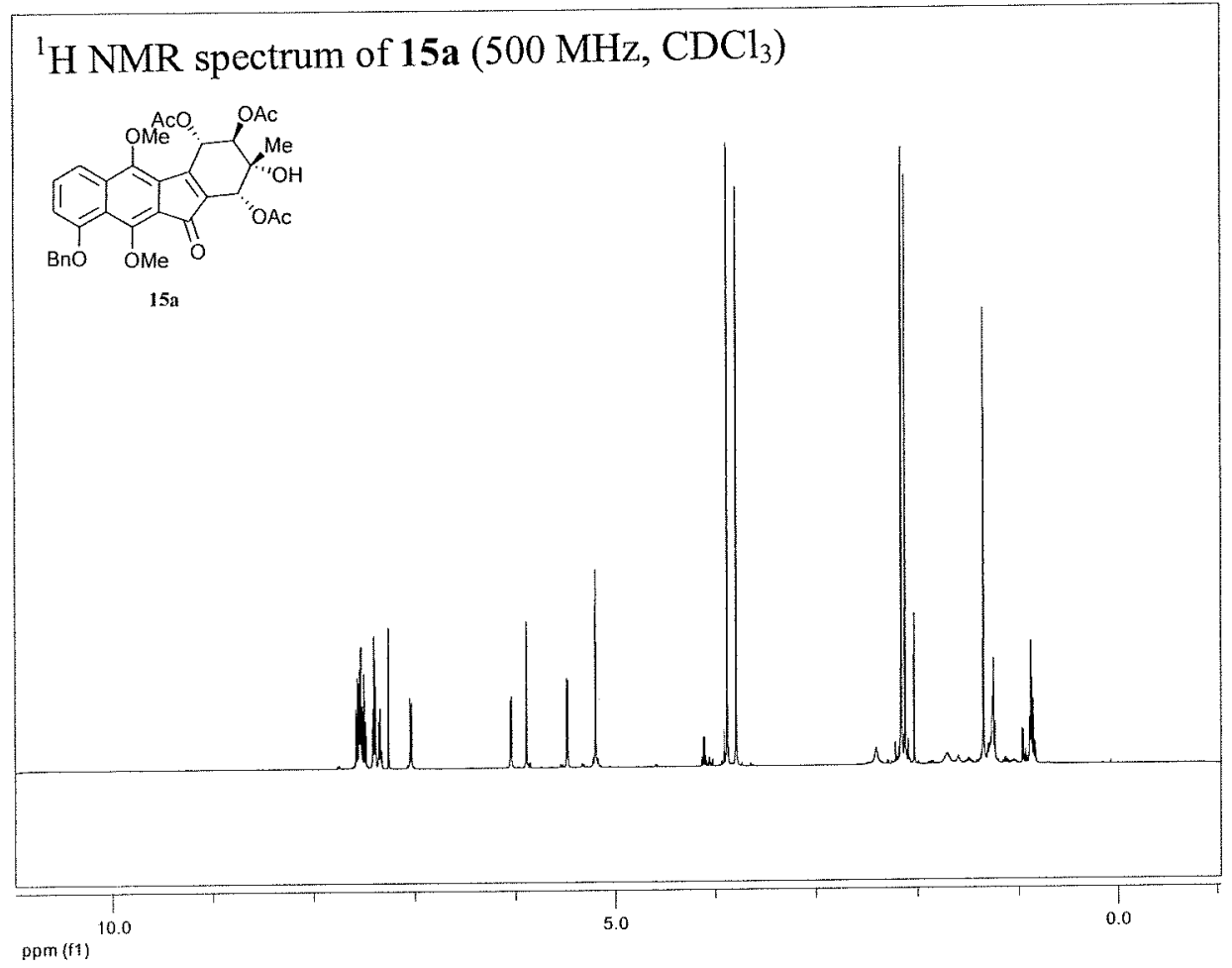

${ }^{13} \mathrm{C}$ NMR spectrum of $\mathbf{1 5 a}\left(125 \mathrm{MHz}, \mathrm{CDCl}_{3}\right)$

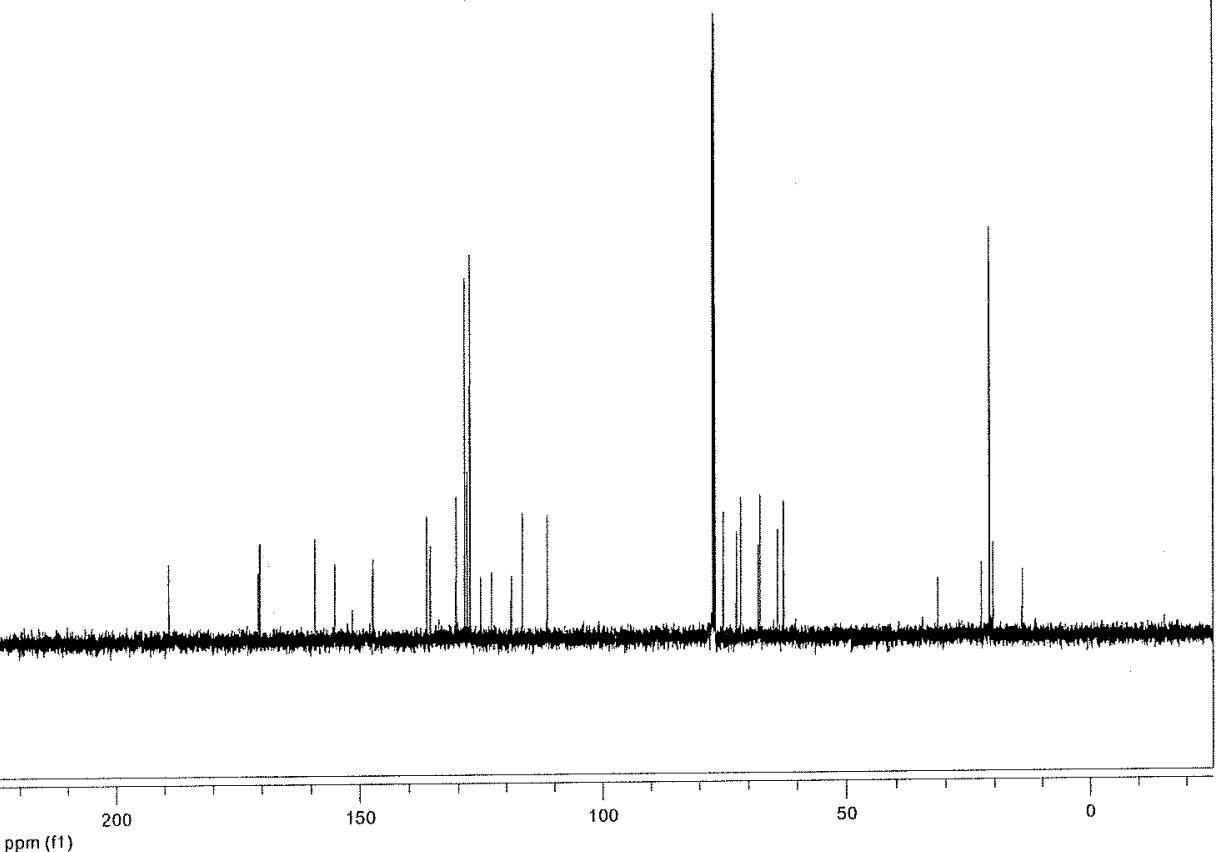




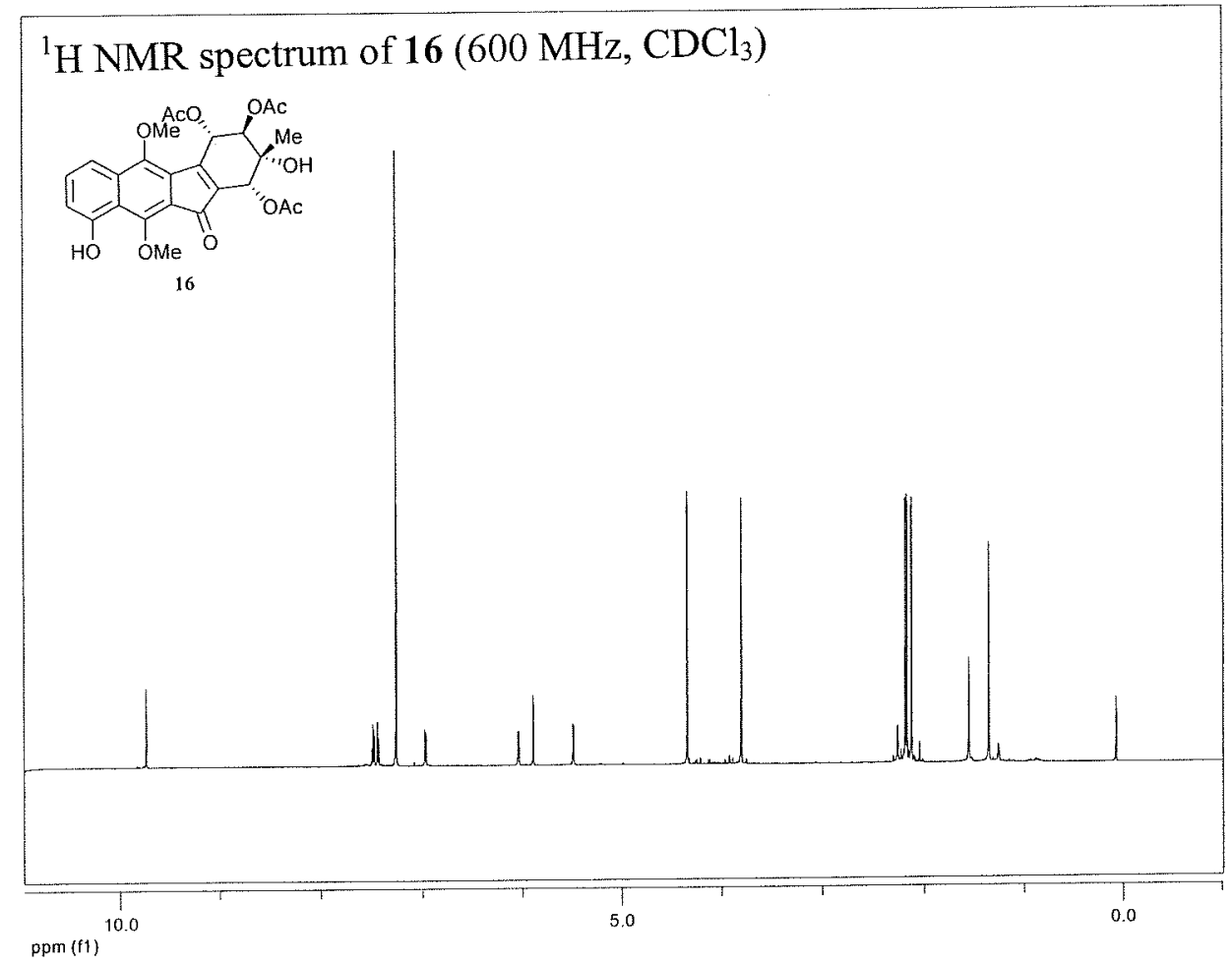

${ }^{13} \mathrm{C}$ NMR spectrum of $16\left(150 \mathrm{MHz}, \mathrm{CDCl}_{3}\right)$

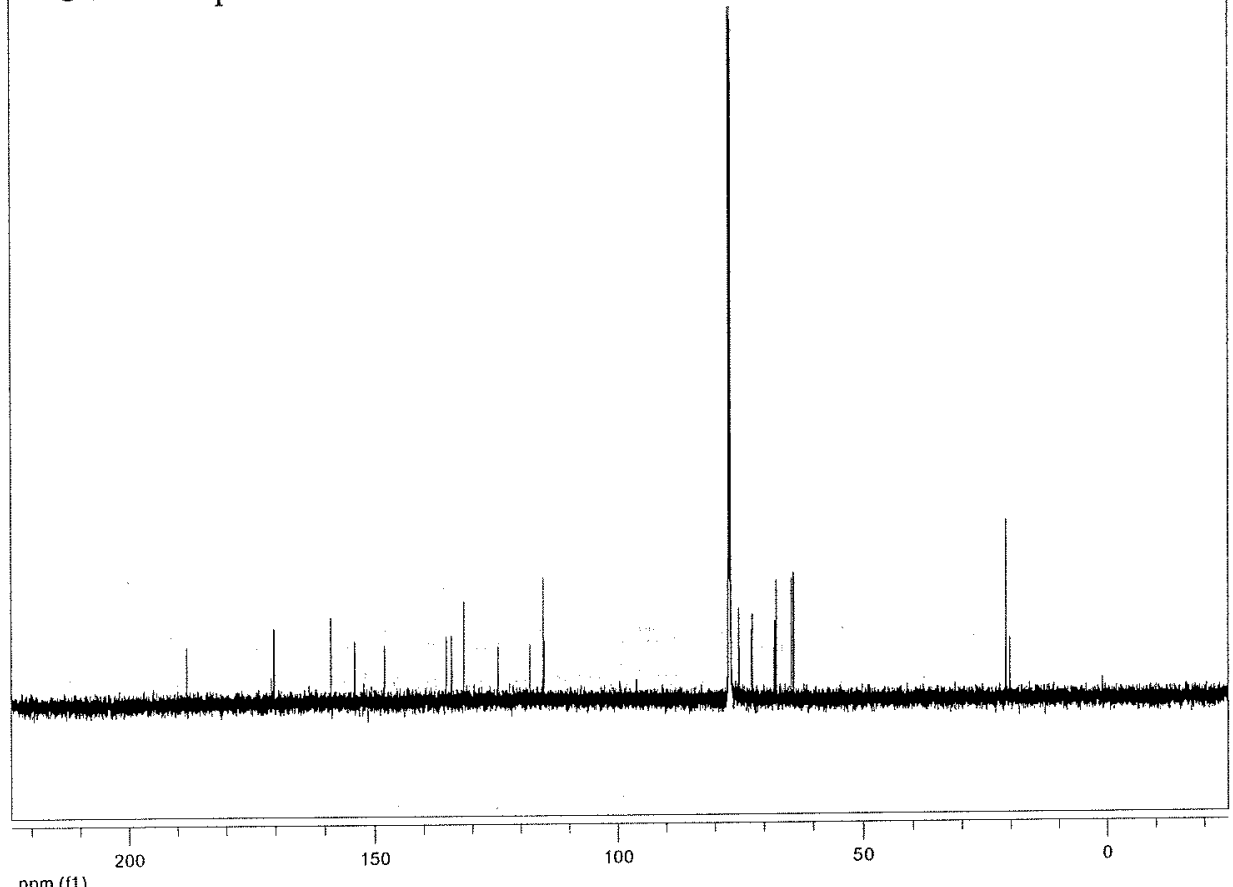



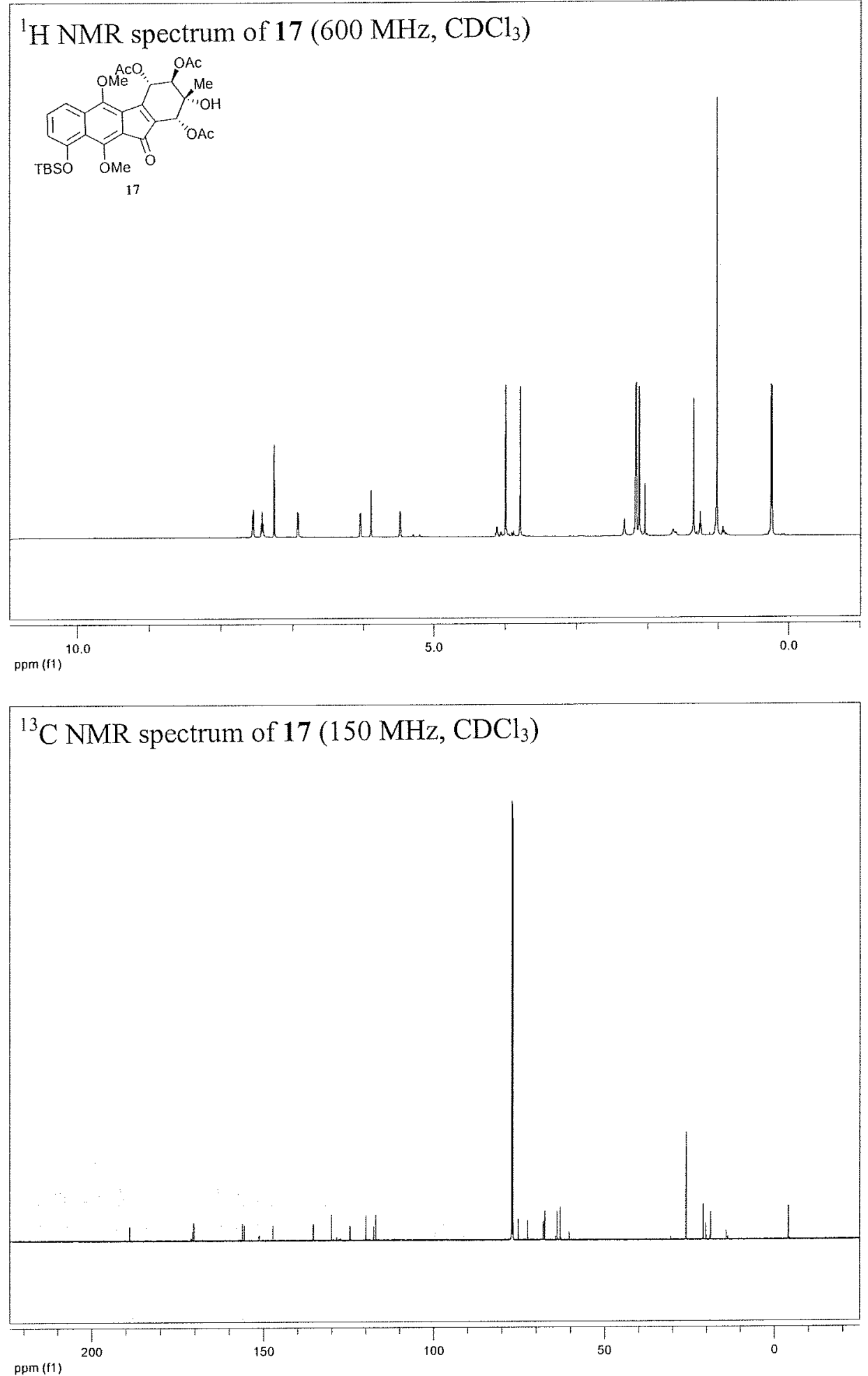


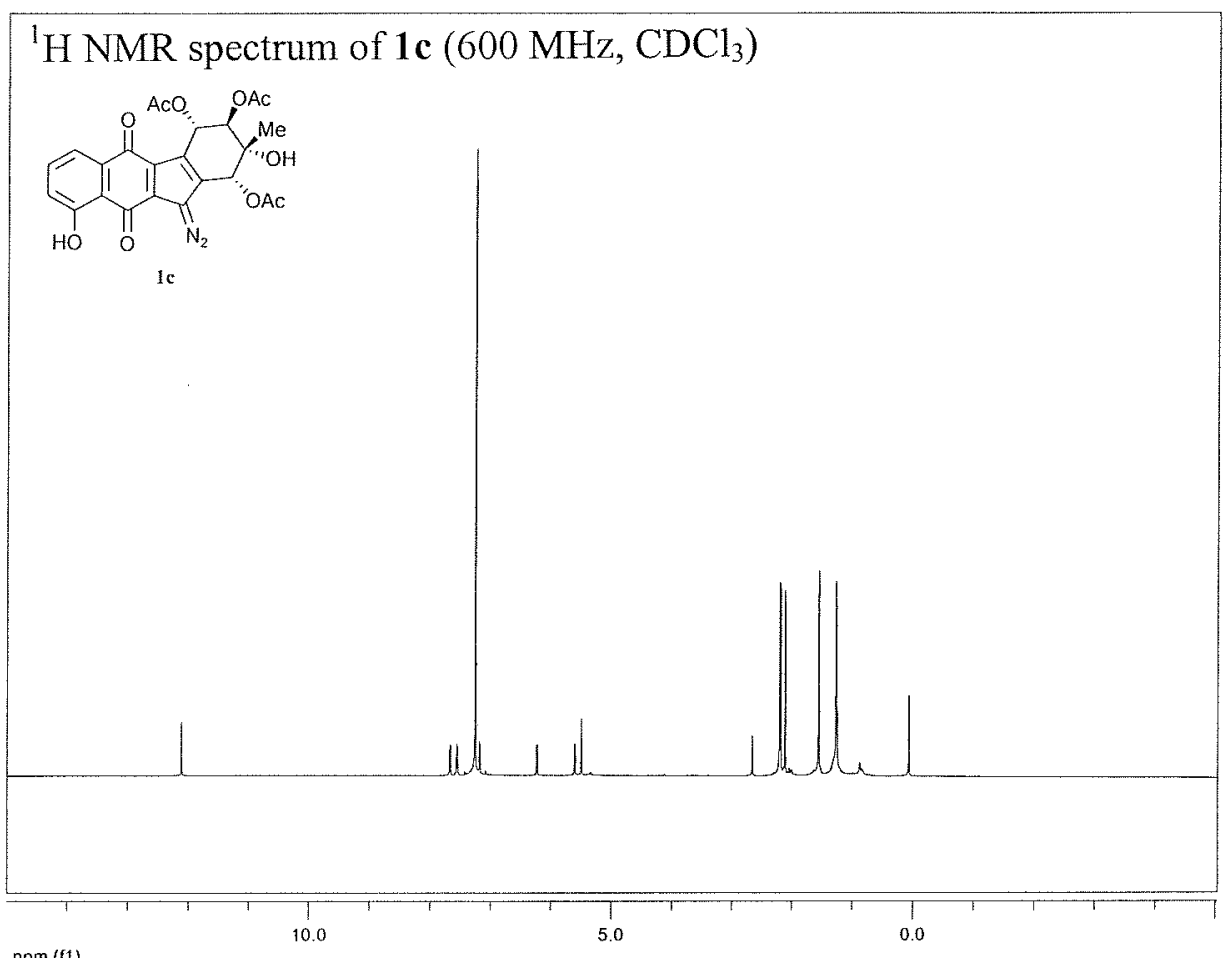

ppm (fi)

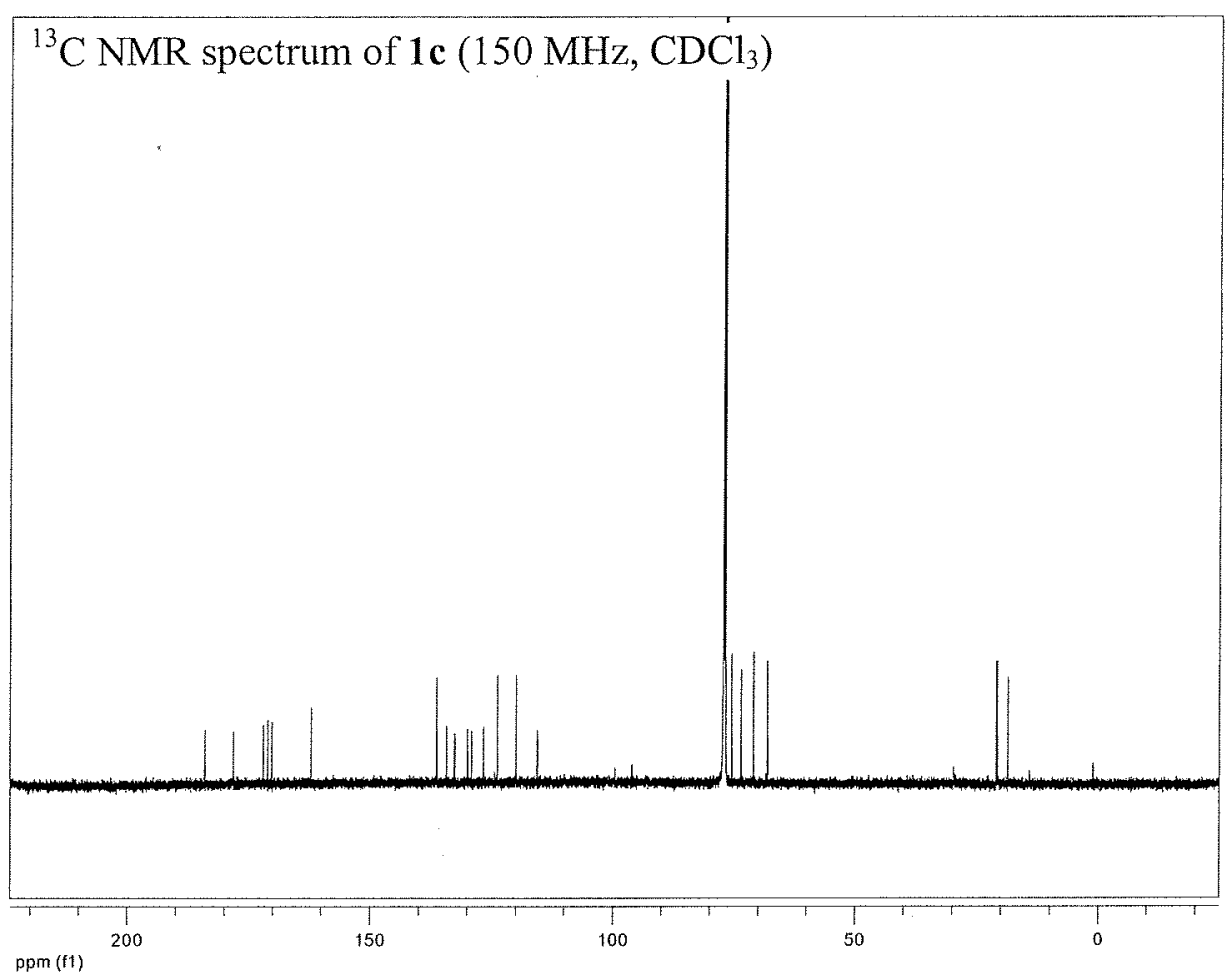




\section{Analyst: Andrea N}

Date Created: Friday, June 08, 2007 9:49 AM Pacific Daylight Time

Spectrum Name: aln-11-C20.sp

Description:

Comments:

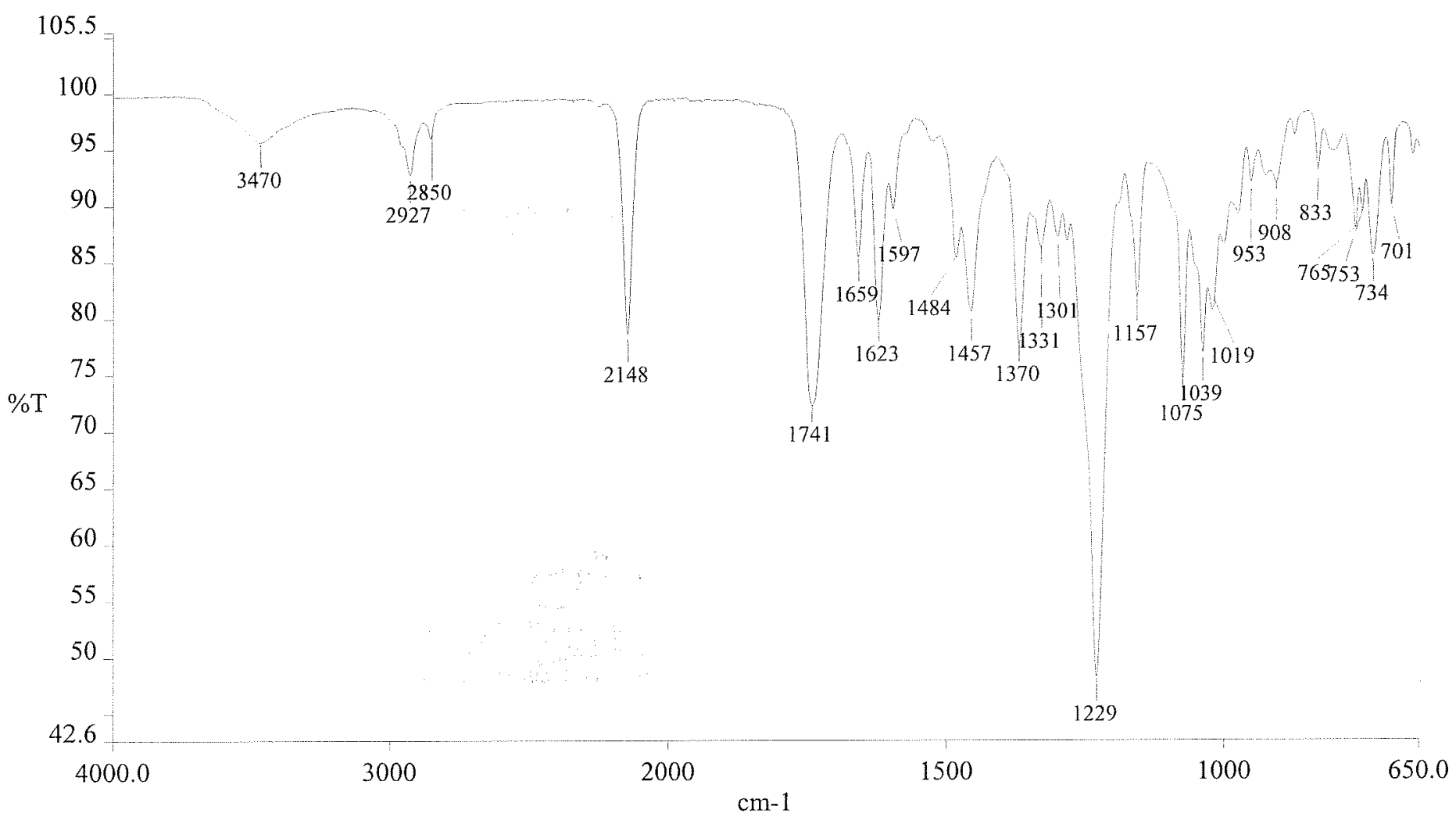

aln-11-C20.sp

aln-11-C20.pk

aln-11-C20.sp $3351 \quad 4000 \quad 6504899 \quad 4 \%$ T 42

REF 400099200099600

$\begin{array}{llllllllll}3470 & 95 & 2927 & 92 & 2148 & 78 & 1741 & 72 & 1659 & 85\end{array}$

$\begin{array}{llllllllll}1623 & 79 & 1597 & 89 & 1484 & 85 & 1457 & 80 & 1370 & 77\end{array}$

$\begin{array}{llllllllll}1331 & 86 & 1301 & 87 & 1229 & 48 & 1157 & 81 & 1075 & 74\end{array}$

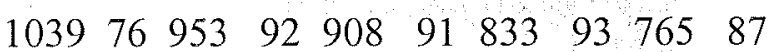

$\begin{array}{llllll}753 & 89 & 734 & 85 & 701 & 90\end{array}$ 

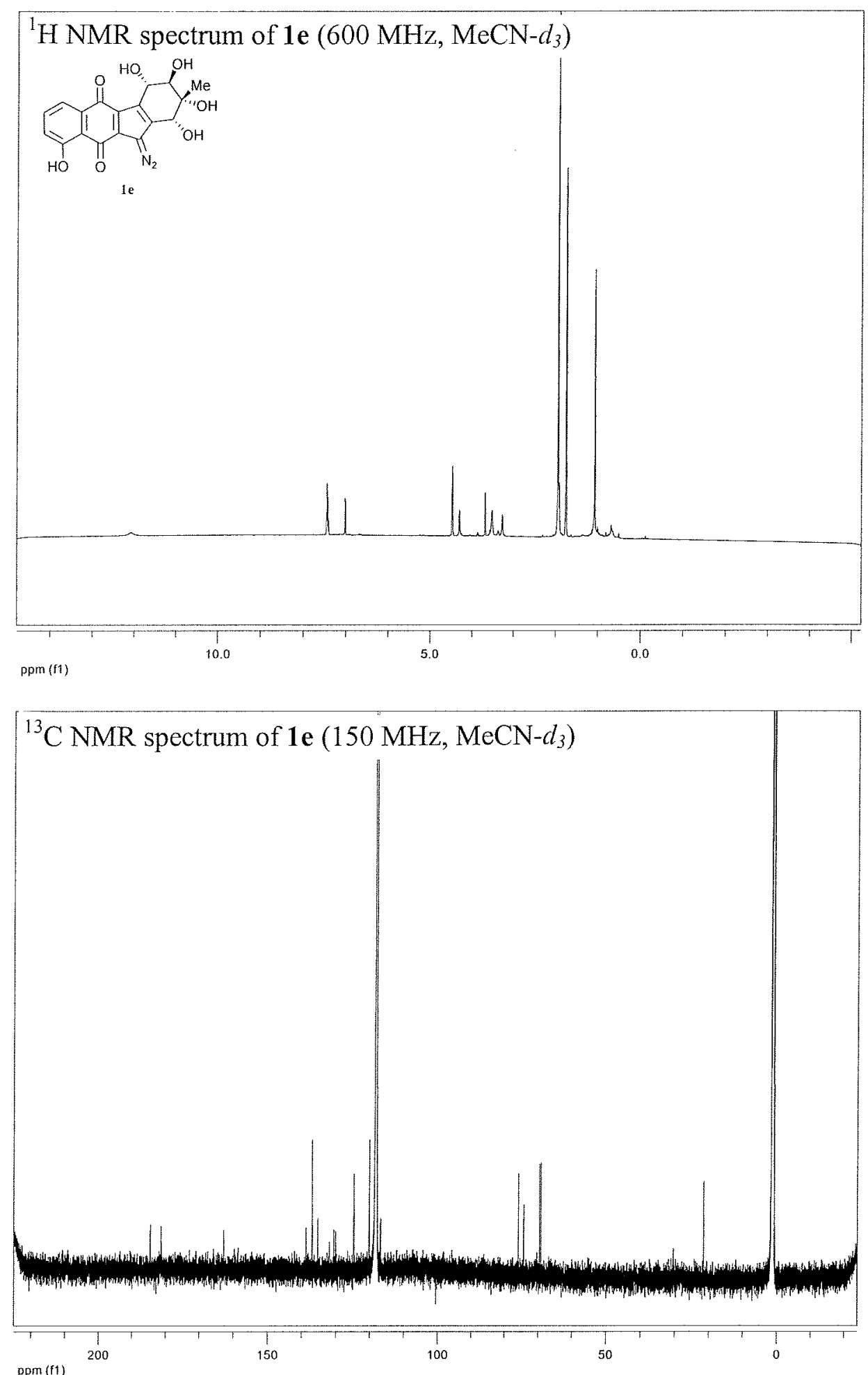


\section{Analyst: Andrea N}

Date Created: Tuesday, June 12, 2007 10:42 AM Pacific Daylight Time

Spectrum Name: aln-11-C21.sp

Description: Kinamycin F

\section{Comments:}

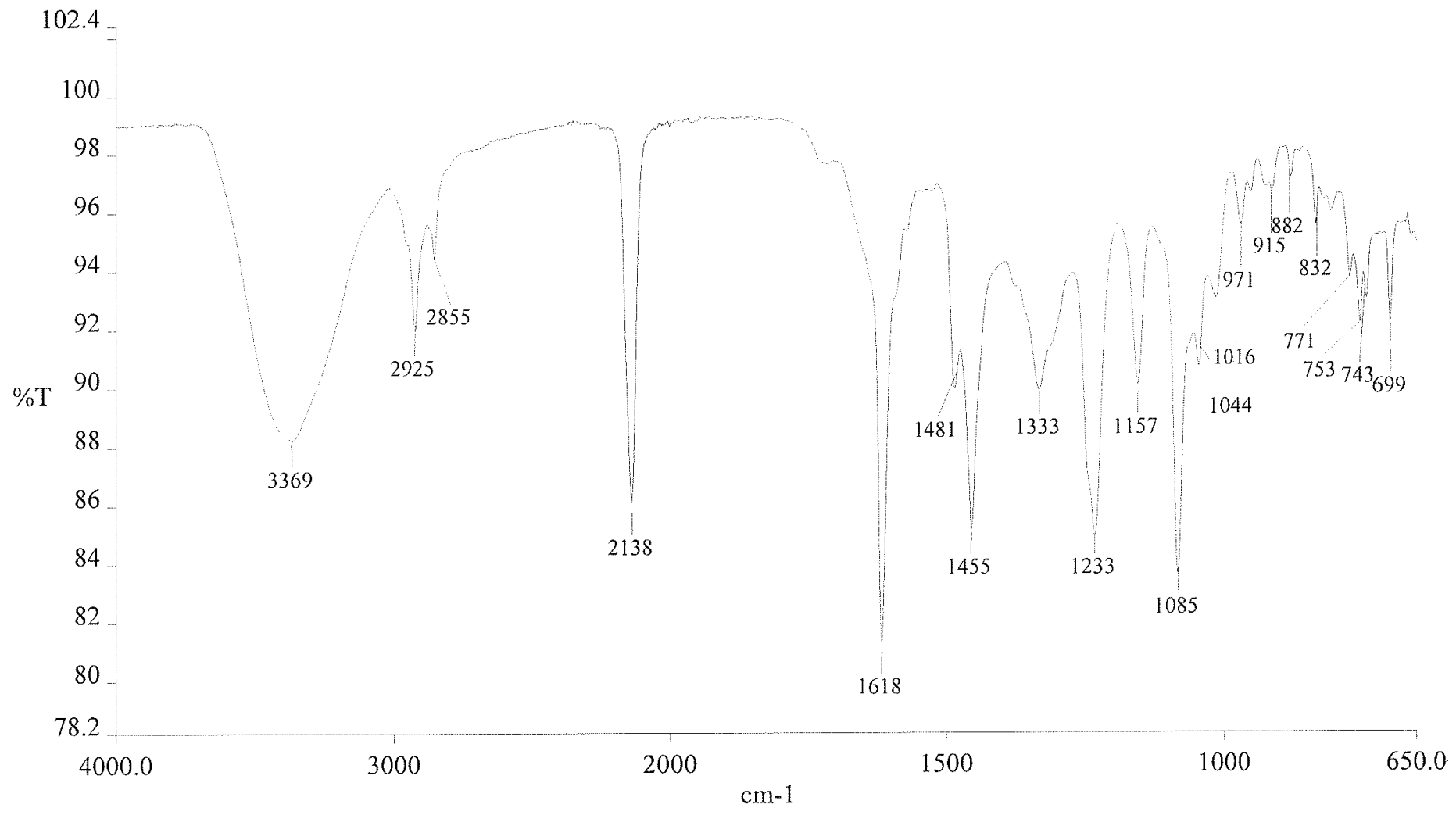

aln-11-C21.sp

aln-11-C21.pk

AL879D 1.SP $3351400065081994 \%$ T 42

Kinamycin F

REF 400099200099600

$\begin{array}{llllllllll}3369 & 88 & 2925 & 91 & 2138 & 86 & 1618 & 81 & 1455 & 85\end{array}$

$\begin{array}{llllllllll}1333 & 89 & 1233 & 84 & 1157 & 90 & 1085 & 83 & 753 & 92\end{array}$

69992 

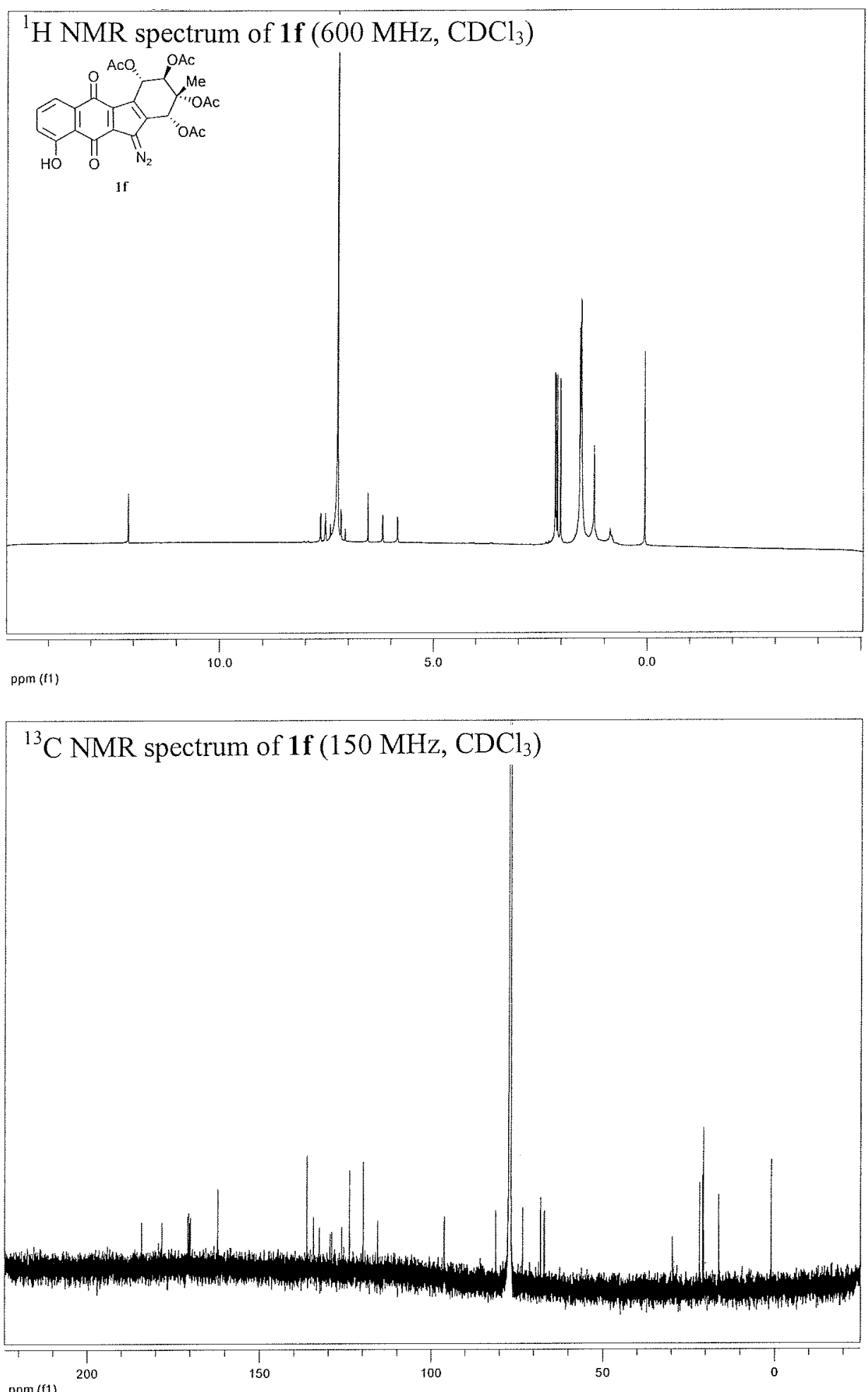
Analyst: Andrea N

Date Created: Tuesday, June 12, 2007 7:18 PM Pacific Daylight Time

Spectrum Name: aln-11-C22-2.sp

Description: Kinamycin J

Comments:

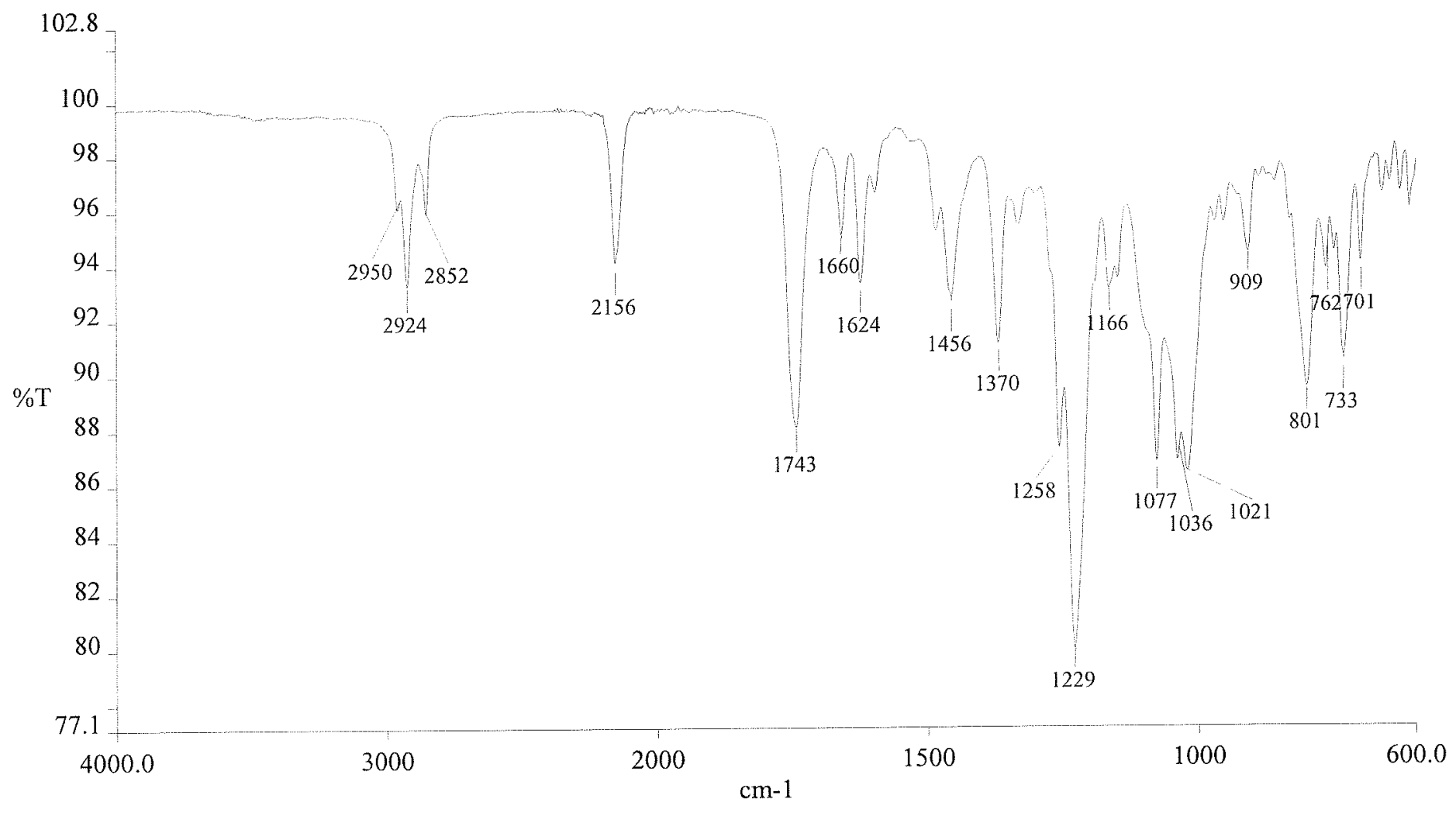

aln-11-C22-2.sp

aln-11-C22-2.pk

aln-11-C22-2.sp 3401400060080994 \%T 42

Kinamycin J

REF 400099200099600

$\begin{array}{lllllllll}2924 & 93 & 2156 & 94 & 1743 & 88 & 166095 & 1624 & 93\end{array}$

$\begin{array}{llllllllll}1456 & 92 & 1370 & 91 & 1258 & 87 & 1229 & 80 & 1166 & 93\end{array}$

$\begin{array}{llllllllll}1077 & 86 & 1021 & 86 & 909 & 94 & 801 & 89 & 733 & 90\end{array}$

70194 